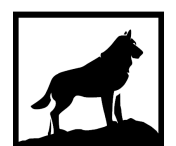

Michigan

Technological



Michigan Technological University

Digital Commons @ Michigan Tech

Dissertations, Master's Theses and Master's Reports

2020

USING TRANSFER PATH ANALYSIS AND FREQUENCY BASED

SUBSTRUCTURING TO DEVELOP A ROBUST VIBRATION

LABORATORY DYNAMIC TEST FIXTURE DESIGN PROCESS

Cora Taylor

Michigan Technological University, corat@mtu.edu

Copyright 2020 Cora Taylor

Recommended Citation

Taylor, Cora, "USING TRANSFER PATH ANALYSIS AND FREQUENCY BASED SUBSTRUCTURING TO DEVELOP A ROBUST VIBRATION LABORATORY DYNAMIC TEST FIXTURE DESIGN PROCESS", Open Access Master's Thesis, Michigan Technological University, 2020.

https://doi.org/10.37099/mtu.dc.etdr/1097

Follow this and additional works at: https://digitalcommons.mtu.edu/etdr

Part of the Acoustics, Dynamics, and Controls Commons 


\title{
USING TRANSFER PATH ANALYSIS AND FREQUENCY BASED SUBSTRUCTURING TO DEVELOP A ROBUST VIBRATION LABORATORY DYNAMIC TEST FIXTURE DESIGN PROCESS
}

By

Cora J. Taylor

\begin{abstract}
A THESIS
Submitted in partial fulfillment of the requirements for the degree of MASTER OF SCIENCE

In Mechanical Engineering
\end{abstract}

MICHIGAN TECHNOLOGICAL UNIVERSITY

2020

(C) 2020 Cora J. Taylor 
This thesis has been approved in partial fulfillment of the requirements for the Degree of MASTER OF SCIENCE in Mechanical Engineering.

Department of Mechanical Engineering-Engineering Mechanics

\author{
Thesis Co-Advisor: Dr. James P. DeClerck \\ Thesis Co-Advisor: Dr. Jason R. Blough \\ Committee Member: $\quad$ Mr. Charles D. Van Karsen \\ Department Chair: $\quad$ Dr. William W. Predebon
}




\section{Table of Contents}

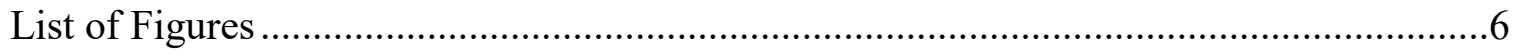

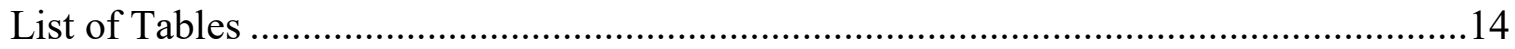

Preface

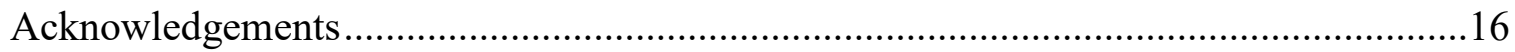





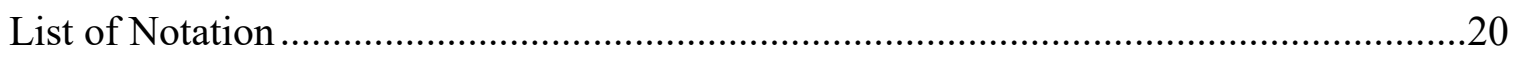

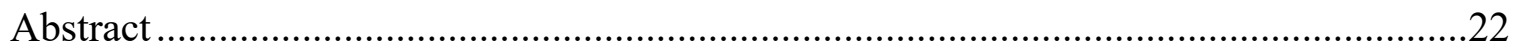

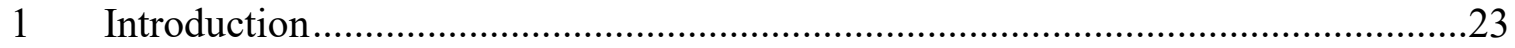

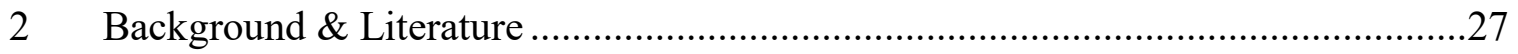

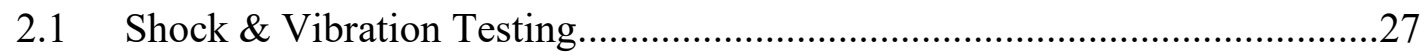

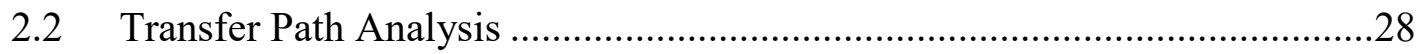

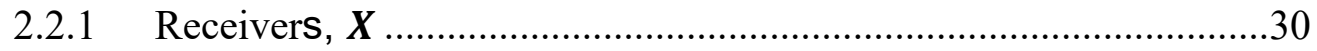

2.2.1.1 Time Response ............................................................30

2.2.1.2 Power Spectral Density ……………………….................31

2.2.1.3 Shock Response Spectrum ................................................32

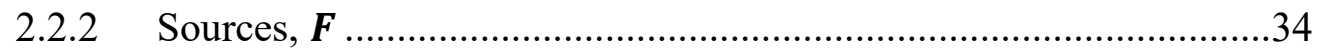

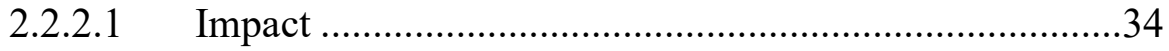

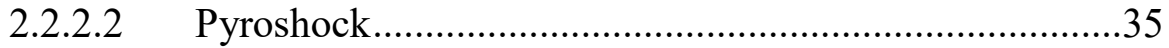

2.2.2.3 Field Data Replication ......................................................36



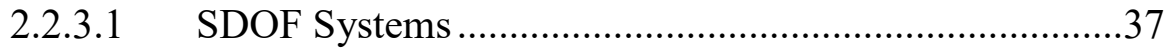


2.2.3.2 MDOF Systems.............................................................

2.2.3.3 Frequency Response Functions.........................................40

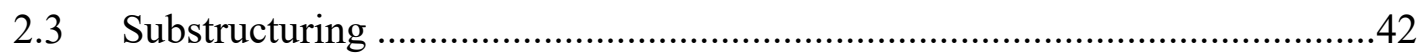

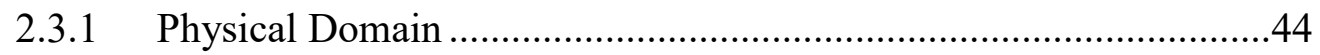

2.3.2 Frequency Domain.........................................................................45

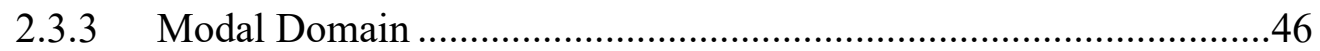

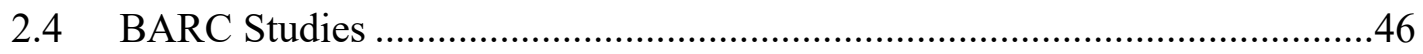

2.4.1 Modal Analysis ..............................................................................

2.4.2 Fixture Optimization FEA …………………...........................49

2.4.3 Multi-Axis Testing........................................................................

3 Theory Applied to BARC ………………………..............................................52

3.1 BARC Frequency Based Substructuring ......................................................52

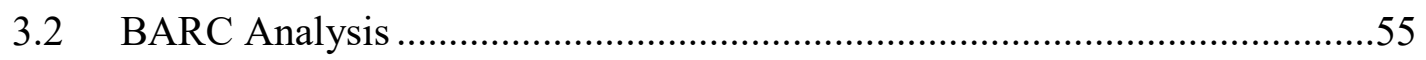

3.2.1 Attachment Dynamics Analysis........................................................56

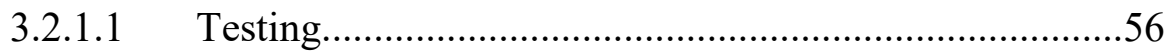

3.3 Component Analysis ...........................................................................5

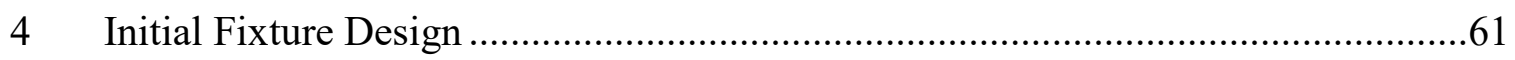

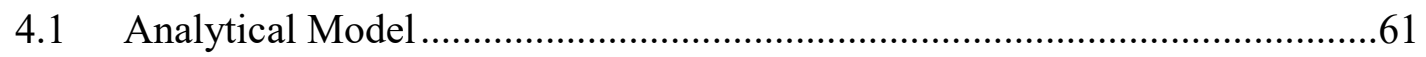

4.1.1 Design Development .........................................................................61

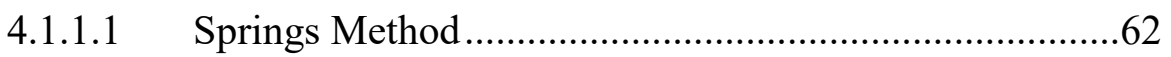

4.1.1.2 Stiffness Analysis..............................................................64

4.1.2 Finite Element Model ………………………...........................66

4.1.3 Hardware ................................................................................

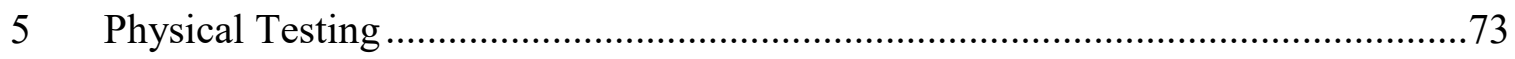

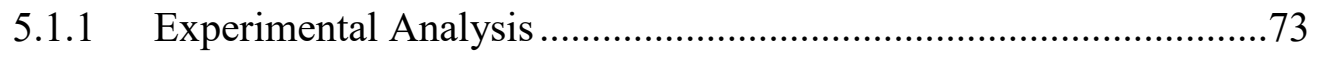

5.1.1.1 Test 1: Attachment FRFs ................................................73 




5.1.1.3 Shaker Voltage Signal.....................................................76

5.1.1.4 Component Response.......................................................77

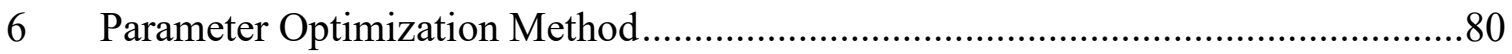

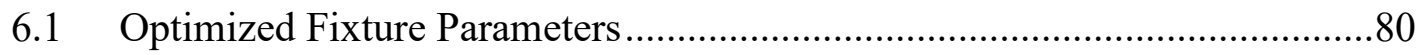





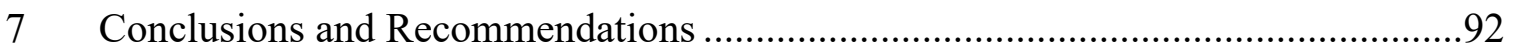

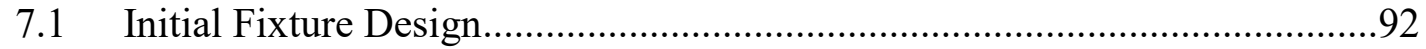

7.1.1 Test 1: Attachment FRFs ........................................................92

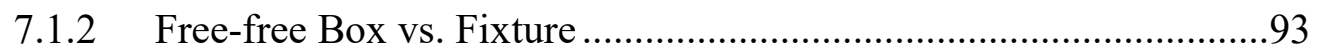

7.1.3 Fixed Box vs. Fixture..............................................................94



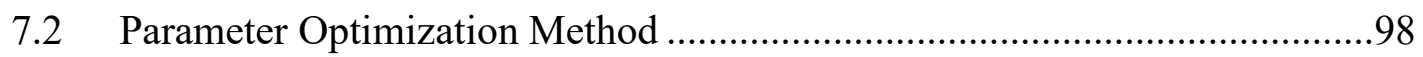





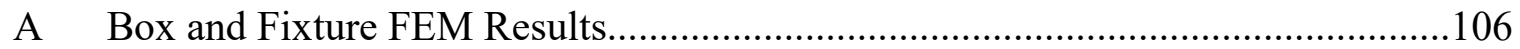

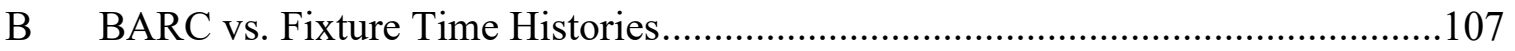




\section{List of Figures}

Figure 1.1 Boundary Condition in Environmental Testing Challenge Problem test bed used to develop a method for laboratory fixture design [1].

Figure 1.2 Schematic of Box Assembly with Removable Component (BARC) and next level assembly described using transfer path analysis (TPA)

Figure 2.1 Schematic of COI in field and laboratory testing environments, where the field environment contains the COI attached to the next-structure and the test environment has the $\mathrm{COI}$ attached to a test fixture.

Figure 2.2 Source-Path-Receiver flow example for an automobile. .29

Figure 2.3 Time response collection from a vibrating structure. .30

Figure 2.4 Power Spectral Density calculation concept map, showing how three signals with different sample rates can be more easily compared with a PSD [7]. 32

Figure 2.5 Development of the SRS from the maximum acceleration of a series of SDOF systems of equivalent damping [8].

Figure 2.6 Example of an impact source of Force (Newton) vs Time (second) .35

Figure 2.7 Schematic of impact source production in the laboratory. 35

Figure 2.8 Single DOF model that is used as the basis to developing equations describing vibration problems.

Figure 2.9 Multiple degree of freedom representation of a skyscraper with an external force.

Figure 2.10 Graphical representation of the Frequency Response Function (Compliance) depicting the mass, damping, and stiffness controlled regions 
Figure 2.11 Schematic of two subsystems before and after substructuring, where point 2 represents the attachment DOFs and interface forces.

Figure 2.12 Dynamic substructuring domains and the conversions necessary between the three.

Figure 2.13 Geometry used for 4 different datasets during modal testing of the BARC from 4 collaborators on the Boundary Condition Challenge Problem [16]

Figure 2.14 FEM used for shape expansion to compare 4 different modal tests performed on the BARC structure [16]. .48

Figure 2.15 FEM expansion from 4 different test geometries shown in Figure 2.13 for the first elastic mode [16].

Figure 3.1 Schematic of the BARC broken into two parts for attachment DOF analysis $\left(A_{1}\right.$ and $\left.A_{2}\right)$, showing external forces $(F B)$ and target data $(X T)$. .52

Figure 3.2 Schematic of the component and fixture for attachment DOF analysis $\left(A_{1}\right.$ and $\left.A_{2}\right)$, showing external forces $(F f)$ and fixture-component response $(X f)$. .54

Figure 3.3 Box from BARC test set-up used to obtain FRF matrices from attachment locations. .56

Figure 3.4 COI from BARC test set-up used to obtain FRF matrices from attachment locations.

Figure 3.5 Accelerance Matrix from Free-Free Box Modal Test used to Determine Stiffness (Y-axis: $\log$ 0.001-1000 g/N, X-axis: Linear 0-3200 Hz) .57

Figure 3.6 FRF and SRS plots comparing three different stiffness's for the next-structure to a rigid and a dynamic test fixture model. .59 
Figure 3.7 Comparison of component attachment accelerance (red) to next-structure (box, blue) attachment accelerance (Y-axis: Log 0.001-1000 g/N, X-axis: Linear 0-3200 $\mathrm{Hz})$

Figure 4.1 Physical Modal Test Performed on AM Parts and Rubber Pillars Mounted to Shaker as Rigid Base .63

Figure 4.2 3-Hole Additively Manufactured Parts (Green) vs. Box (Blue) (Y-axis: Log 0.001-1000 g/N, X-axis: Linear 0-3200 Hz)

Figure 4.3 Rubber Bars (Green) vs. Box (Blue) (Y-axis: Log 0.0001-1000 g/N X-axis: Linear 0-3200 Hz). .64

Figure 4.4 Simple schematic of dynamic test fixture design based on connecting spring concept, a connection between the attachment locations is necessary to produce a full FRF matrix. .64

Figure 4.5 Load case defined for connecting spring, $\mathrm{k}_{3}$, rubber block: $\mathrm{Y}$ (Compression/Tension), X (Shear), Z (Shear).

Figure 4.6 Finite Element Model of the connecting spring with specified loading and constraint conditions. .68

Figure 4.7 Displacement results and stiffness Ratio from the Finite Element Analysis performed on the proposed model of $\mathrm{k}_{3}$

Figure 4.8 Original dynamic test fixture design as a result of the stiffness values retrieved from the attachment locations on the free-free box.

Figure 4.9 Finite Element Models of Box in free-free condition and dynamic fixture design with fixed base constraints.

Figure 4.10 Box vs. Fixture (Mode 10 vs. Mode 7) where we see the difference in frequency but overall similarity in motion .70 
Figure 4.11 Y-directional accelarance plots from the fixture (Orange) and box (Blue)....71

Figure 4.12 Final fixture design, with AM brackets, rubber pillars, and rubber block in the center.

Figure 5.1 Attachment FRF collection test for next-structure and dynamic test fixture...73

Figure 5.2 Attachment time response collection test for free-free box with random base input. .74

Figure 5.3 Next-structure (box) test set-up used to gather attachment point time histories $(X 1 B$ and $X 2 B)$ from both a random and rocket vibration profile with base control.

Figure 5.4 Fixture test set-up used to gather attachment point time histories $(X 1 f$ and $X 2 f)$ from both a random and rocket vibration profile with base control.

Figure 5.5 Next-structure test set-up used to obtain the voltage signal going to the shaker from controlling at the attachment locations with time response signals from the previous test.

Figure 5.6 Fixture test set-up used to obtain the voltage signal going to the shaker from controlling at the attachment locations with time response signals from the previous test.

Figure 5.7 Obtaining the component response from free-free box with base input. .78

Figure 5.8 Next-level assembly test set-up to gather component response based off voltage input gathered from controlling an attachment location based on attachment time histories.

Figure 5.9 Fixture assembly test set-up to gather component response based off voltage input gathered from controlling an attachment location based on attachment time histories. 
Figure 5.10 Component instrumentation for both target data and fixture response collection

Figure 6.1 Test set-up for gathering attachment FRFs for a single attachment next-structure



Figure 6.2 BARC Y-direction single attachment FRFs for LPM development. .81

Figure 6.3 Measured and synthesized Y-direction FRFs from the single attachment nextstructure.

Figure 6.4 Lumped parameter model used to determine the parameters needed to match the target attachment FRF. .83

Figure 6.5 Optimizer iterated FRFs from fixture LPM of single attachment nextstructure.

Figure 6.6 Fixed-fixed beam with tuned absorbers to represent the LPM of the fixture...86

Figure 6.7 Finite Element Model of fixture LPM from optimized parameters. .87

Figure 6.8 Comparison of attachment FRFs between the target, optimized, and FEM.....88

Figure 6.9 Single attachment response recording from shaker excitation of nextstructure.

Figure 6.10 Single attachment response recording from shaker excitation of LPM fixture design.

Figure 6.11 Using attachment time history as waveform replicated control shaker input to obtain shaker voltage drive signal for single attachment next-structure. .90

Figure 6.12 Using attachment time history as waveform replicated control shaker input to obtain shaker voltage drive signal for single attachment LPM fixture. 90 
Figure 6.13 Target response collection from voltage drive signal for uncut BARC........91

Figure 6.14 Component response on LPM test fixture from voltage drive signal............91

Figure 7.1 Accelerance of the Box (Blue) Fixed and the Fixture (green) Fixed (Y-axis: Log 1e-5-1000 N/m X-axis: Linear 0-3200 Hz)

Figure 7.2 Free-free BARC vs. fixture time response at point 1 with RMS, variance, range, and maximum values for each signal.

Figure 7.3 Component response comparison on fixture vs. BARC at point 1 with field data input.

Figure 7.4 Fixed BARC vs. fixture SRS from point 1 on component for 3 identified shock events.

Figure 7.5 Impact test set-up used to gather the attachment FRFs from the next-structure in its surrogate field environment.

Figure 7.6 Lumped Parameter Model of next-structure, used for optimization of parameters to match attachment FRFs.

Figure 7.7 Measured vs. synthesized attachment FRFs from the cut box of the BARC structure. .100

Figure 9.1 X-directional Accelerance Plots from the Fixture (Orange) and Box (Blue). 106

Figure 9.2 Z-directional Accelerance Plots from the Fixture (Orange) and Box (Blue). 106

Figure 9.3 Free-free BARC vs. fixture time response at point 2 with RMS, variance, range, and maximum values for each signal.

Figure 9.4 Free-free BARC vs. fixture time response at point 3 with RMS, variance, range, and maximum values for each signal. 
Figure 9.5 Free-free BARC vs. fixture time response at point 4 with RMS, variance, range, and maximum values for each signal.

Figure 9.6 Free-free BARC vs. fixture time response at point 5 with RMS, variance, range, and maximum values for each signal.

Figure 9.7 Free-free BARC vs. fixture time response at point 6 with RMS, variance, range, and maximum values for each signal.

Figure 9.8 Component response comparison on fixture vs. BARC at point 2 with field data input. 110

Figure 9.9 Fixed BARC vs. fixture SRS from point 2 on component for 3 identified shock events. 110

Figure 9.10 Component response comparison on fixture vs. BARC at point 3 with field data input

Figure 9.11 Fixed BARC vs. fixture SRS from point 3 on component for 3 identified shock events.

Figure 9.12 Component response comparison on fixture vs. BARC at point 4 with field data input.

Figure 9.13 Fixed BARC vs. fixture SRS from point 4 on component for 3 identified shock events.

Figure 9.14 Component response comparison on fixture vs. BARC at point 5 with field data input.

Figure 9.15 Fixed BARC vs. fixture SRS from point 5 on component for 3 identified shock events. 113 
Figure 9.16 Component response comparison on fixture vs. BARC at point 6 with field data $i_{p}$

Figure 9.17 Fixed BARC vs. fixture SRS from point 6 on component for 3 identified shock events. 


\section{List of Tables}

Table 1.1 Definitions of the parts present in the Boundary Condition Challenge test

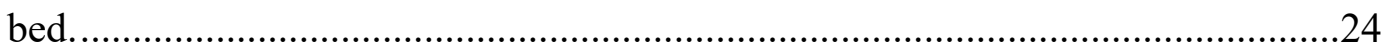

Table 2.1 Forms of the frequency response function (FRF) equation.

Table 4.1 Stiffness values in $\mathrm{kN} / \mathrm{m}$ from Box FRF matrix to be used to design the dynamic test fixture.

Table 4.2 Directional stiffness values collected from free-free Box impact test in kN/m.65

Table 4.3 Stiffness ratio of connecting spring, $\mathrm{k} 3$, used to begin initial dynamic test fixture design .65

Table 4.4 Required stiffness and length values for the three sides of the connecting "spring" for the dynamic test fixture design to produce similar stiffness to the box.

Table 6.1 Optimization targets from single attachment target FRF .82

Table 6.2 Optimizer iterated values for the tuned absorber parameters from fixture LPM of single attachment next-structure.

Table 6.3 Results of 3 tests to determine which type of BCs the main beam should have. .86

Table 6.4 Lumped Parameter Model tuned absorber mass and stiffness properties .87

Table 7.1 Free-free BARC vs. fixture time response comparison metrics values and differences.

Table 7.2 Fixed BARC vs. Fixture component time response comparison metrics values. 


\section{Preface}

The work presented is research conducted at Michigan Technological University. It was funded through the Department of Energy's Kansas City National Security Campus, operated and managed by Honeywell Federal Manufacturing \& Technologies, LLC under contract number DE-NA0002839. 


\section{Acknowledgements}

Over the years I have been lucky enough to have many friends, family members, and mentors to guide me throughout my college career, however I never thought I would finish without any of them around during a pandemic. Nonetheless, thank you to my advisors and mentors for always being available to answer my questions, and point me in the right direction. Thank you to my friends for supporting me, mostly from afar, and getting my mind off the craziness that is completing a master's degree. Finally, thank you to my family for being just a phone call away me grounded and smiling when things got really tough. 


\section{Definitions}

Attachment impedance: Impedance present at the locations where a component of interest attaches to a structure.

Box: Aluminum sub-assembly designed to act as the "vehicle" or next-level structure to produce field boundary conditions.

Component: The device under test (DUT), or component of interest (COI) also defined as the removable component in the BARC structure, two C-channels with a connecting beam.

Field configuration: The assembly of the component and subassembly in its designed assembled state (BARC).

Field environment: The dynamics the component experiences during its intended use, in this case when the component is attached to the box with some external forces applied to the box.

Honeywell: Department of Energy's Kansas City National Security Campus, operated and managed by Honeywell Federal Manufacturing \& Technologies, LLC.

Impedance: A frequency response function (FRF) that is defined by velocity over force.

Impedance mismatch: The difference between the next-structure and laboratory test fixture impedance.

Laboratory environment: The dynamics the component experiences during a laboratory test, in this thesis this is when the component is connected to a fixture and some force is applied to said fixture.

Next-structure: The sub-assembly the component of interest is attached to in its field configuration. 
Next-level assembly: The assembly of the next-structure and the component of interest, also known as the field configuration.

Test fixture: The piece of hardware to which the component is attached to in the laboratory configuration, the interface between the component and the test equipment.

Undertest: When a laboratory test of the component of interest does not meet the dynamics of the service environment, therefore leading to a false pass.

Overtest: When a laboratory test of the component of interest does not meet the dynamics of the service environment, therefore leading to a false failure. 


\section{List of Abbreviations}

BARC: Box Assembly with Removable Component

$\underline{\mathrm{COI}}$ : Component of Interest

DOF(s): Degree(s) of Freedom

DUT: Device Under Test

FBS: Frequency Based Substructuring

FEA: Finite Element Analysis

FEM: Finite Element Model

FRF: Frequency Response Function

IMMAT: Impedance-Match Multi-Axis Testing

LPM: Lumped Parameter Model

MDOF: Multiple Degree of Freedom

MTU: Michigan Technological University

PCA: Principle Component Analysis

PSD: Power Spectral Density

RMS: Root Mean Square

SDOF: Single Degree of Freedom

SRS: Shock Response Spectrum

SVD: Singular Value Decomposition 


\section{List of Notation}

A: Attachment

$\mathrm{A}_{\mathrm{i}}^{\mathrm{p}}:$ Attachment DOFs

B: Box or subassembly

C: Component

f: Fixture

H: Frequency Response Function

$\overline{\mathrm{F}}$ : External Forces

j: Imaginary unit

M, C, K / m, c, k: Mass, damping, and stiffness

$\mathrm{n}$ : Total number of DOFs

$\mathrm{V}_{\mathrm{B}}$ : Shaker voltage profile for BARC

$V_{f:}$ Shaker voltage profile for fixture

$\mathrm{X}$ : X-DOFs

$\mathrm{X}_{\mathrm{i}}^{\mathrm{p}}$ : Free response

$\overline{\mathrm{X}}_{\mathrm{T}}$ : Target Response (Truth Data)

$\overline{\mathrm{X}}_{\mathrm{f}}$ : Fixture Response

$\widehat{X}$ : Shaker control reference (waveform replicated data) 


\section{Brackets}

[P]: Matrix

$\overline{\mathrm{P}}$ : Vector

\section{$\underline{\text { Operators }}$}

$\mathrm{P}^{\mathrm{T}}$ : Transpose

$\mathrm{P}^{-1}$ : Inverse

\section{$\underline{\text { Superscripts \& Subscripts }}$}

$\mathrm{R}_{\mathrm{i}}$ : Attachment location 1 or 2

$\mathrm{Rp}$ : Structure specification of component (C), Box/subassembly (B), or fixture (f)

HxA: FRFs from attachment to X-DOFs

$\mathrm{H}_{\mathrm{AA}}$ : Attachment FRFs

$\mathrm{H}_{\mathrm{AF}}$ : Free-Response FRFs 


\section{Abstract}

The objective of this research is to determine a robust method for designing shock and vibration laboratory test fixtures that accurately reproduce the field environment for the component of interest. The methods presented in this research examine the boundary conditions presented unto the component of interest through transfer path analysis with the intent of reproducing the next-structure attachment impedance with a laboratory test fixture of one and two attachments. The first method includes only using the next-structure relative motion and stiffness to design a test fixture, while the second method uses an optimization algorithm to develop a lumped parameter model that produces equivalent attachment FRFs. Both methods are conducted and validated using the Box Assembly with Removable Component structure with two attachment locations fashioned by the Department of Energy's Kansas City National Security Campus, operated and managed by Honeywell Federal Manufacturing \& Technologies, LLC and Sandia National Laboratories. 


\section{Introduction}

Determining if a component or structure will fail in its field environment is a necessity for many industries. One of the ways to ensure that the component of interest (COI) will achieve its expected life is to conduct laboratory tests. The design and execution of a vibration or shock test requires planning to ensure that the test produces the results the engineer is looking to achieve. The goal of a laboratory test is to replicate the dynamic environment that the COI experiences in the field. The interest is to reproduce the force input and boundary conditions (BCs) into the $\mathrm{COI}$ with the test equipment and a test fixture to observe component response.

In an effort to motivate collaboration of the dynamic environmental testing community around the effectiveness of shock and vibration test fixtures, the Department of Energy's Kansas City National Security Campus, operated and managed by Honeywell Federal Manufacturing \& Technologies, LLC and Sandia National Laboratories developed The Boundary Condition Challenge. This challenge was intended to assist in producing a new methodology to follow when designing laboratory test fixtures, by being able to design a component-level test setup that allows the COI to undergo a similar environmental exposure that it experiences in the next-level assembly (field configuration) [1]. Figure 1.1 and Table 1.1 define the test bed used to complete this work.

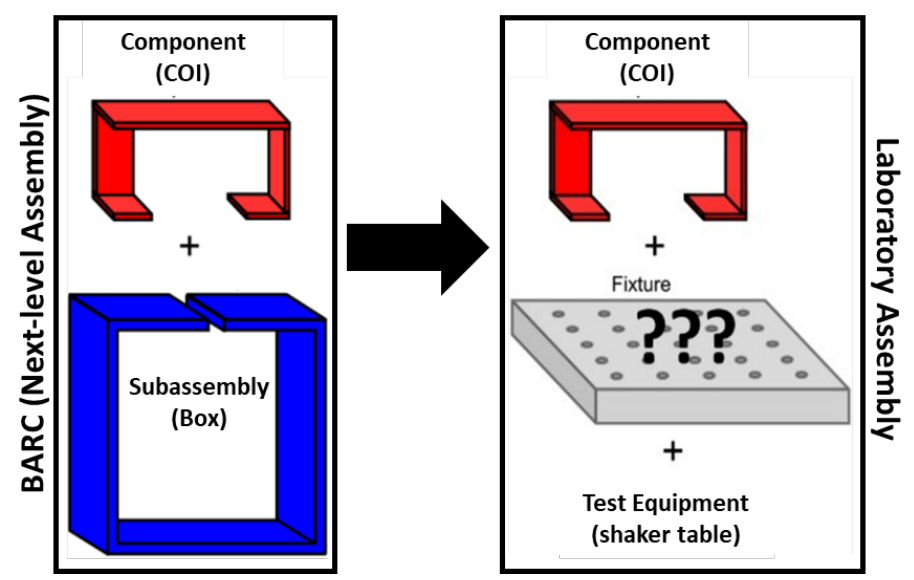

Figure 1.1 Boundary Condition in Environmental Testing Challenge Problem test bed used to develop a method for laboratory fixture design [1]. 
Table 1.1 Definitions of the parts present in the Boundary Condition Challenge test bed.

Subassembly: The vehicle or "next-structure," in this case it is the Box seen in blue in Figure 1.1.

Component: Component of interest (COI), the structure in which we are concerned with its response in the field and laboratory environments, seen in red in Figure 1.1.

BARC: $\quad$ Box Assembly with Removable Component, combination of the component and subassembly seen on the left side of Figure 1.1.

Fixture: $\quad$ The interface between the test equipment and the component that reproduces component field conditions in the laboratory, the combination is the test surrogate for the subassembly.

MTU was responsible for fabricating, assembling, and shipping BARC samples to members of the shock and vibration community following the introduction of the challenge at IMAC XXXVI in 2018, and has been using the structure and involved in the challenge since.

The BARC is the next-level assembly, where we are able to obtain the goal response at the receiver (COI) from a particular input or source. Our goal is to design a structure, to attach to a shaker table, and a shake amplitude profile to produce the same response at the receiver (COI). Figure 1.2 depicts the BARC structure used throughout this work and a design space for the new structure in a transfer path analysis (TPA) form. The source is the forces acting on the structure, while the box is the path and the component is the receiver. The goal is to replace the path with a different structure, and achieve the same response at the receiver (COI), meaning $\bar{X}_{T}=\bar{X}_{f}$. 

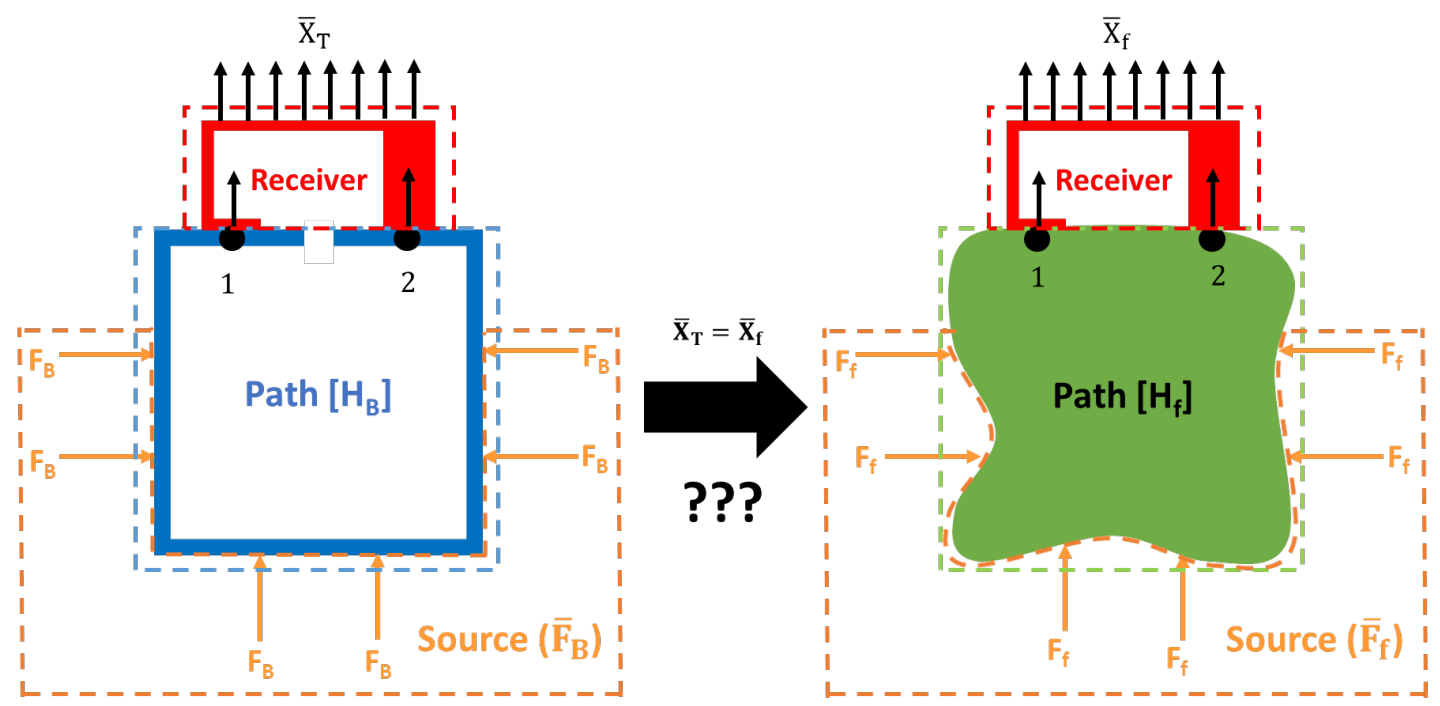

Figure 1.2 Schematic of Box Assembly with Removable Component (BARC) and next level assembly described using transfer path analysis (TPA).

The next-level assembly and test assembly can be described with Equations 2.1.1 and 2.1.2.

$$
\begin{aligned}
& \bar{X}_{T}=\left[H_{B}\right] \bar{F}_{B} \\
& \bar{X}_{f}=\left[H_{f}\right] \bar{F}_{f}
\end{aligned}
$$

Where $\bar{F}_{B}$ and $\bar{F}_{f}$ represent the external forces presented unto the next-level and laboratory assemblies, respectively. The subscript $\mathrm{T}$ represents the next-level or "target" response, the subscript B stands for the box of the BARC, and the subscript $f$ depicts the fixture or lab structure. A successful laboratory test is defined to have the same response as the field environment as described by Equation 2.1.3.

$$
\bar{X}_{T}=\bar{X}_{f}
$$

In understanding TPA, we can factually say that if the transfer functions are equivalent between the two structures cases, the response at the receiver will be alike. In the frequency domain we use the FRF to define the dynamics of a structure. If a test fixture can be designed to have an equivalent FRF matrix to the next-level assembly's FRF matrix, then 
a source will exist for the laboratory test that will produce an identical response on the COI in the lab as it sees in the field [2]. 


\section{Background \& Literature}

This chapter begins with a brief discussion and history of shock and vibration testing and analysis, starting from the fundamentals. In addition, there is a discussion of transfer path analysis and the importance of understanding the source, path and receiver of a system. Then, attachment impedance and its usefulness in fixture design is reviewed. Additionally, the chapter concludes with substructuring in three domains. Finally, it discusses the BARC structure and some studies performed by the dynamic environment testing community using it.

\subsection{Shock \& Vibration Testing}

Formal vibration testing came into existence following World War II [3]. It was on the forefront of the engineering world at this time due to physical systems exposed to dynamic loads failing in unexpected ways. This was a result of failure modes often being defined under static loads [3]. The war stimulated innovation, and the need for vibration qualification testing, to ensure equipment would perform as expected in its operational environment [4].

Dynamic testing is defined as the measurement and analysis of a structure's dynamic response as it endures a defined shock and/or vibration environment. Vibration is a repetitive application of forces, while shock is a high amplitude, short duration, high frequency event presented unto a structure.

Shock and vibration qualification testing is often conducted by attaching the COI to a fixture and applying some forcing profile to it (Figure 2.1). Field environment sources often contain a combination of random vibration, shock events, and potentially impact events. These profiles are reproduced in the laboratory in many ways including shaker, impact, or even acoustic excitations. However, sometimes accurately reproducing the field vibration source is not enough to sufficiently replicate in the test configuration, the forces experienced by the COI in its field environment. 




Figure 2.1 Schematic of $\mathrm{COI}$ in field and laboratory testing environments, where the field environment contains the $\mathrm{COI}$ attached to the next-structure and the test environment has the $\mathrm{COI}$ attached to a test fixture.

\subsection{Transfer Path Analysis}

Transfer path analysis (TPA) is defined as a test- or simulation- based procedure allowing one to trace the flow of vibro-acoustic energy from a source, through a structure or air transfer path, and provided to the receiver where the signal is recorded [5]. Paths in TPA are described by transfer functions. These transfer functions are defined in the LaPlace domain, but can be realized in the frequency domain as functions of the mass, stiffness and damping of the path, such as a FRF [2]. The response of receiver or component is the product of the source force and the system transfer functions or FRF matrix. Equation 2.2.1 is the common equation used to describe TPA, while the following definitions explain the three parts required for TPA.

$$
\bar{X}=[H] \bar{F}
$$


Source, $\bar{F}$ :

Quantitative and qualitative description of energy or force entering the system.

Path, $[H]$ : Mechanism that transmits (gain/delay) energy from the source to the receiver.

Receiver, $\bar{X}: \quad$ Quantitative and qualitative description of how the output energy is observed.

An easily relatable example of TPA is when you feel a vibration or hear a noise in your vehicle cabin. The vibration could be a result of multiple sources (e.g. motor, road surface, wheel unbalance), transmitted through your car frame/body or through the air and received by you feeling the vibration or hearing the noise. Figure 2.2 shows a schematic of this example.
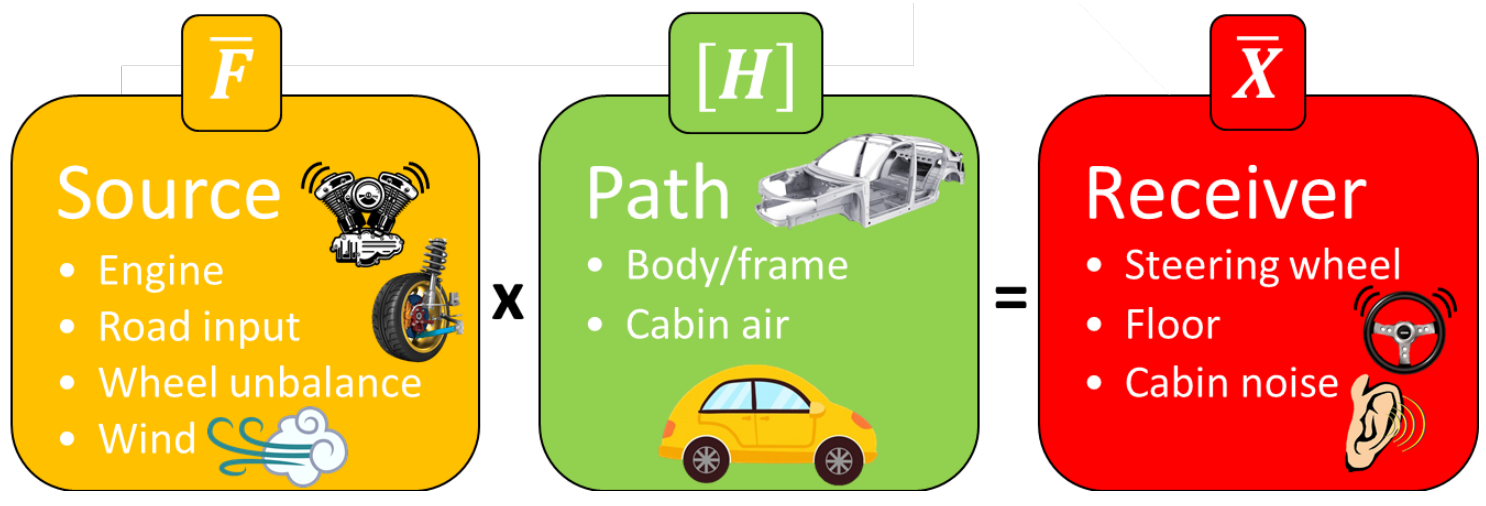

Figure 2.2 Source-Path-Receiver flow example for an automobile.

All shock and vibration analysis can be broken down into its sources, paths, and receivers. The goal of TPA is to evaluate the contribution along each potential path to the receiver to identify what modifications need to be made where to achieve a certain result, or solve a present problem. Since the path can attenuate, amplify or delay the energy coming from the source, it can be difficult to identify where the modifications are necessary. With respect to shock and vibration testing we can use TPA to research if there is a way we can build another structure with the same path to achieve the same results at the receiver in different configurations, the laboratory and the field. Depending on the problem, a solution 
may be present in modifying the source and/or path, and the response can be viewed in different manners based on calculations performed at the receiver.

\subsubsection{Receivers, $\bar{X}$}

When performing a vibration test, the desired dynamic response of the COI is often defined. It is important to first understand what response is desired, and how to achieve and characterize that response. There are many methods used to characterize a structures dynamic response, with each method revealing something different about the signal.

\subsubsection{Time Response}

Time response is defined as the dynamic response, usually acceleration, velocity, or displacement, of a component with respect to time. All vibration events are originally measured as a function of time and then converted to a different domain such as frequency. Once in another domain, additional calculations may be performed to achieve the desired receiver characterization. A time response is obtained by attaching a transducer to the COI, and measuring its response as a result of some input. Figure 2.3 shows the process of collecting a time response.

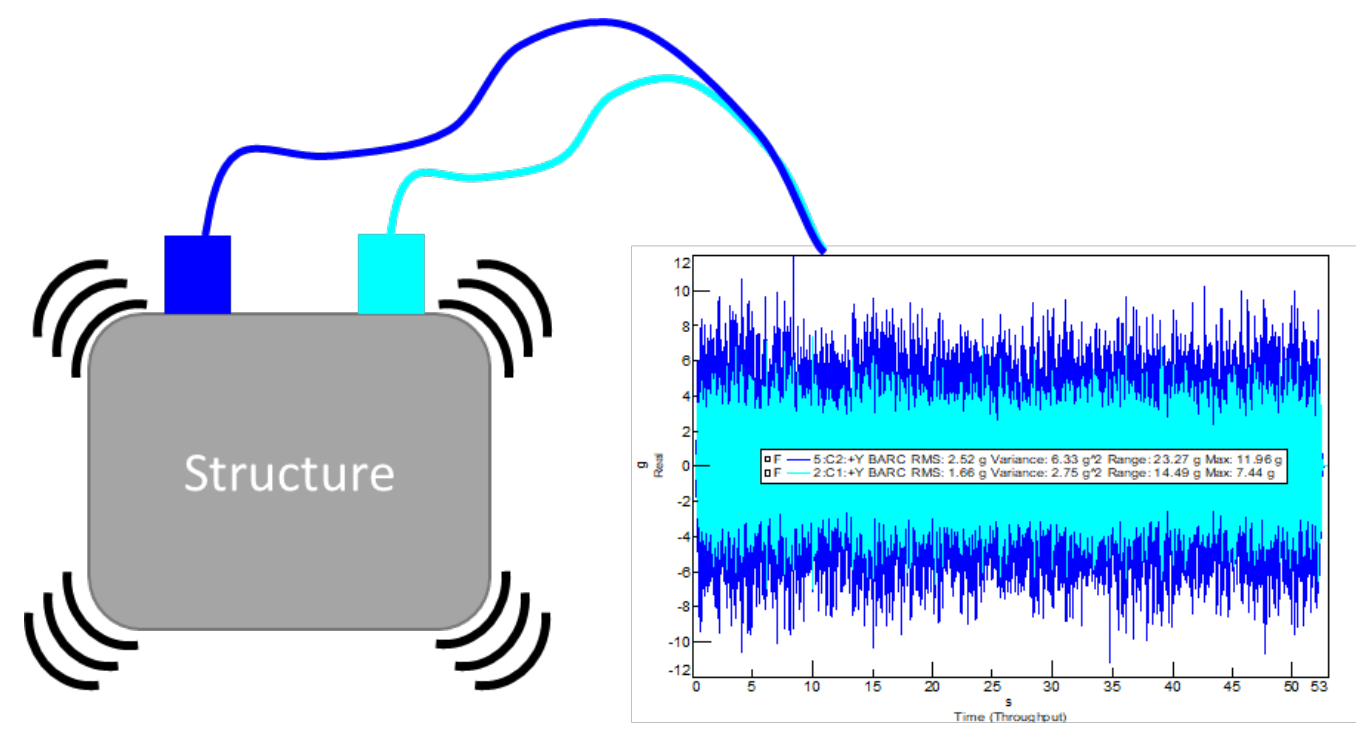

Figure 2.3 Time response collection from a vibrating structure. 
Time-histories generally don't provide much insight into a structures dynamic response without additional calculations, but they are often used as sources for laboratory testing, as will be discussed in section 2.2.2.3 Field Data Replication.

\subsubsection{Power Spectral Density}

The power spectral density (PSD) is another method used to characterize and analyze a vibration event. A PSD is normally used in the characterization of broadband random signals, like those often recovered from field tests. It is defined as the measurement of a signal's power content (magnitude squared) per unit frequency. The units of the function are $E U^{2} / \mathrm{Hz}$, where $E U$ is the engineering unit of acceleration, velocity, or displacement [6]. A PSD is often used to calculate the total power in a frequency band or for comparing different broadband spectra. It is a normalization of the autopower spectrum with respect to the effective bandwidth of the measurement, so different broadband measurements can be compared independently [7]. Figure 2.4 shows a schematic of the concept behind the PSD [7]. 

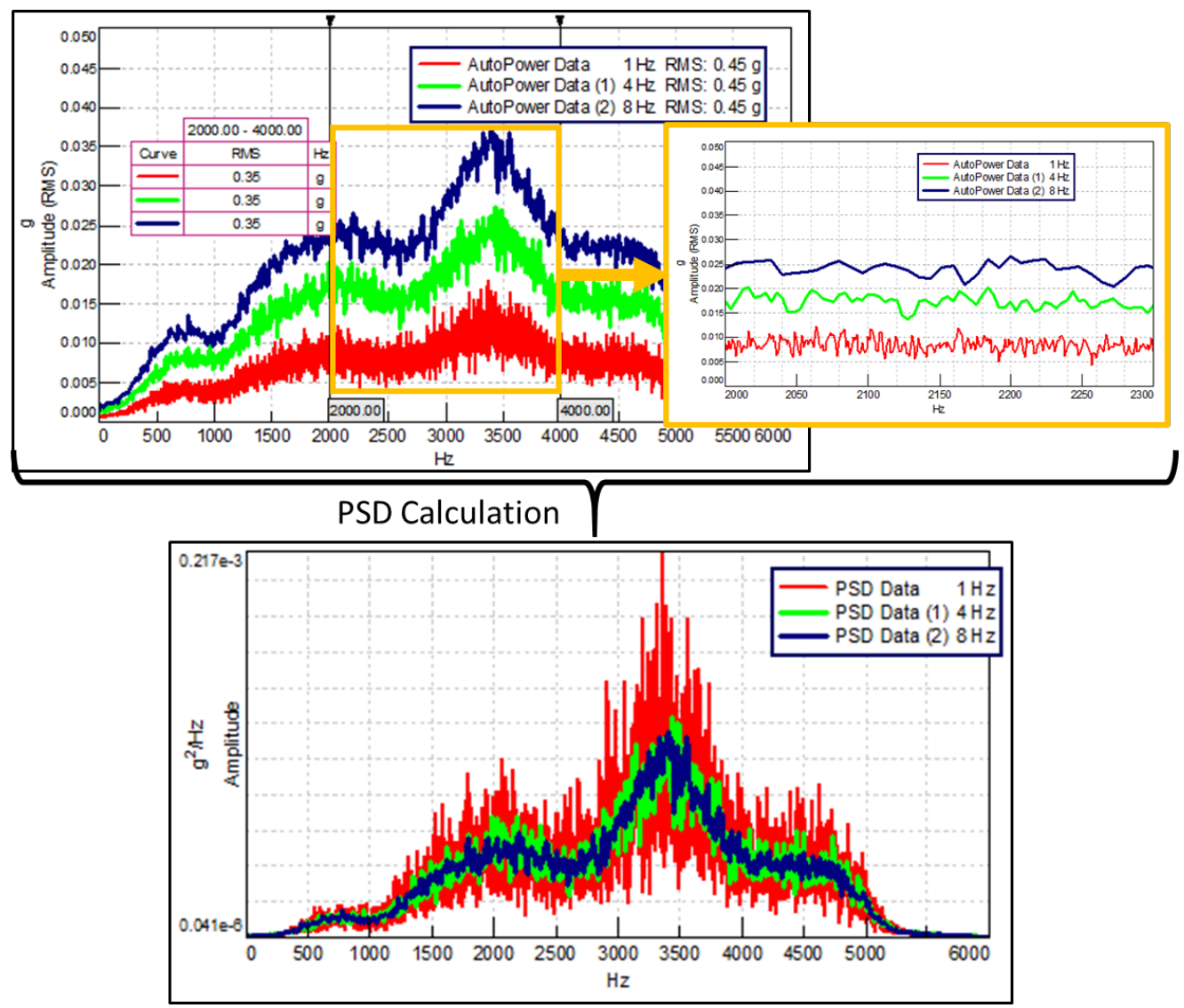

Figure 2.4 Power Spectral Density calculation concept map, showing how three signals with different sample rates can be more easily compared with a PSD [7].

In Figure 2.4 we see three signals (blue, green, and red) with different sample frequencies. These three signals contain the same energy as shown by the RMS values in the top left plot, however it is difficult to compare them due to the differing sample frequencies. The PSD calculation is performed and the bottom center figure is produced, allowing us to more easily see the differences in the three signals regardless of sample frequency.

\subsubsection{Shock Response Spectrum}

The purpose of a shock response spectrum (SRS) is to estimate the maximum dynamic response of a structure through the characterization of the frequency response of the shock environment [8]. A shock environment is produced by a sudden acceleration or deceleration due to a large external force acting on a structure which is usually short in 
time and transient. The SRS is defined mathematically through the analysis of the maximum response of a number of single degree of freedom (SDOF) systems with different natural frequencies as a result of some shock input [8]. The SDOF systems can only translate or rotate around one axis at a time.

Figure 2.5 shows how the SRS is developed using SDOF systems. The damping ratio in all of the systems is equivalent. We imagine that the transient shock input acts on the relatively massive and rigid base, exciting the SDOF systems [8]. The acceleration response of the systems is recorded and the maximum from each system is identified. Each maximum is mapped in the frequency domain at its respective SDOFs natural frequency to create the SRS, for a maximum SRS estimation.

Several events can be characterized by the SRS, and different environments are results of different types of shock events. Earthquakes, naval shock, and Pyroshock are the typical events characterized by the SRS [8]. The SRS is commonly used in standards for designing structures to withstand earthquakes, and components exposed to potential damage due to naval shock and/or Pyroshock.
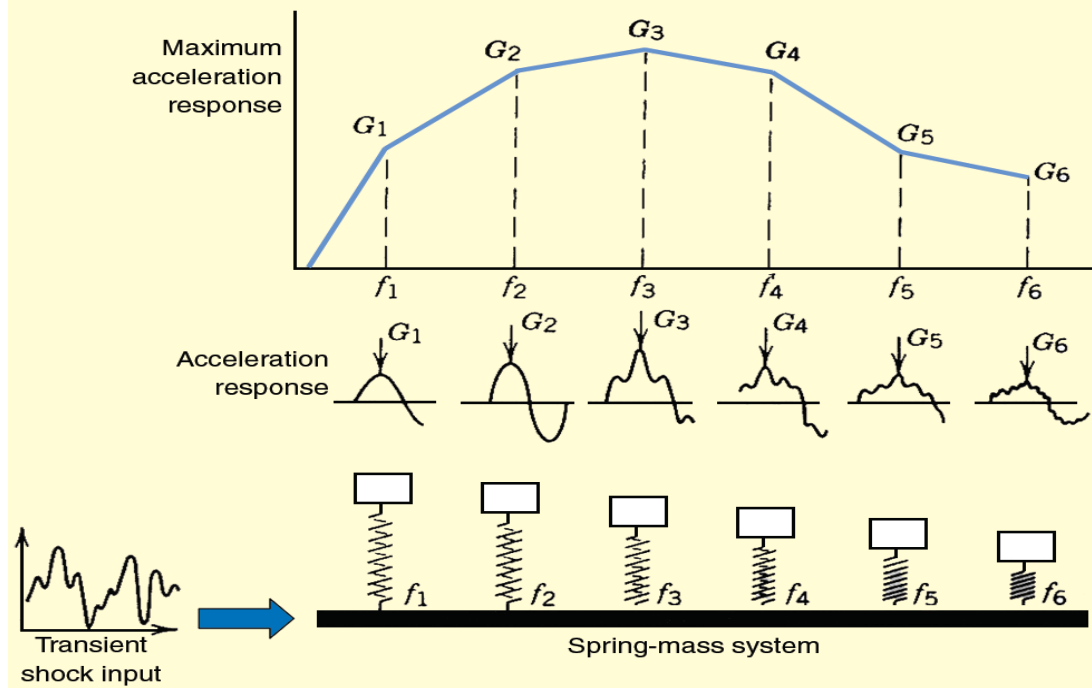

Figure 2.5 Development of the SRS from the maximum acceleration of a series of SDOF systems of equivalent damping [8]. 
Although the SRS can be a useful metric in standard creation, it does have one major limitation. Any given SRS can be produced by many different time histories. Even though the SRS is the same, and satisfies the required standard, this does not mean the shock events are equivalent. A shock event of less severity as the one used in the standard could produce the same SRS as specified by the standard. Hence, components could be over or undertested and still meet the specification.

\subsubsection{Sources, $\bar{F}$}

When beginning a vibration study it is important to first understand what type of source we are dealing with. The purpose of identifying the source is to get an idea of what the response might look like. Different sources can be used with the intent of obtaining different responses. These sources can be random or deterministic. Deterministic excitations are able to be described at any instant in time, meaning they can be generated by a specific mathematical relationship. Random signals are unable to be defined by a mathematical relationship. These random signals can include shock and/or vibration characteristics, and are what is often experienced by a structure in its field environment [9].

\subsubsection{Impact}

Impact testing is a method commonly used to excite a structure with a goal of acquiring FRFs for experimental modal testing [9]. Impact is described as a short duration impulse applied to a structure. Figure 2.6 shows what an impact force input source may look like. 


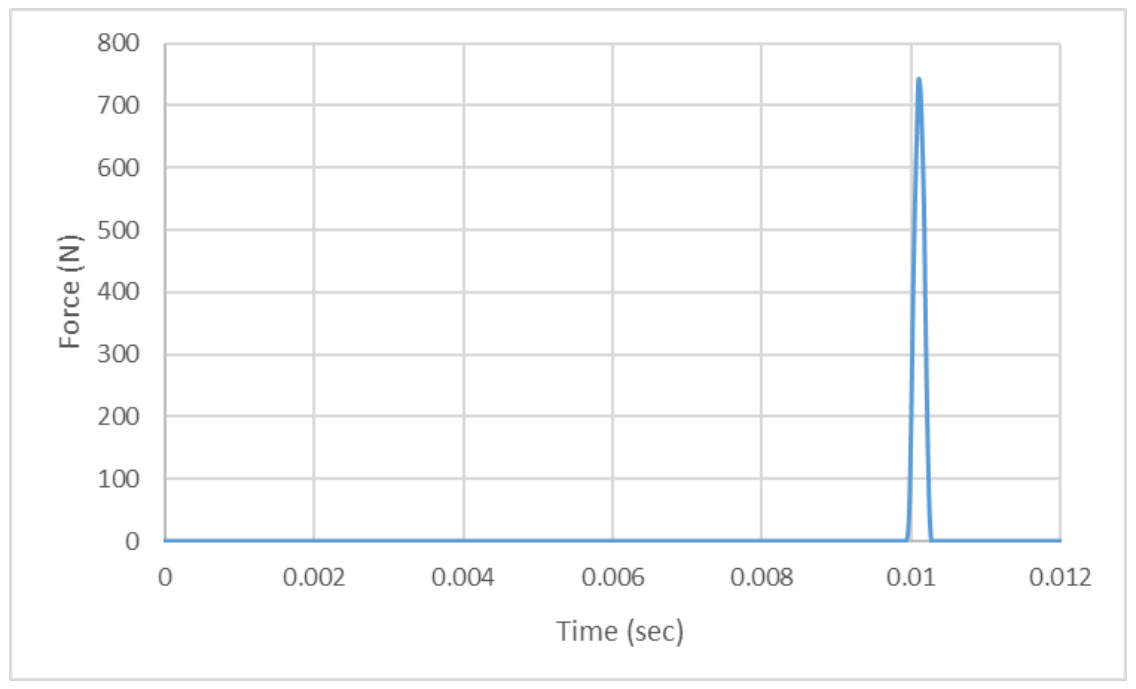

Figure 2.6 Example of an impact source of Force (Newton) vs Time (second) .

Often times, an impact source is produced and recorded in the lab using an impact hammer. The hammer is equipped with a load cell that retrieves a voltage signal that is converted to the force applied to the surface it impacts. Figure 2.7 shows a schematic of how an impact source may be produced in the lab.

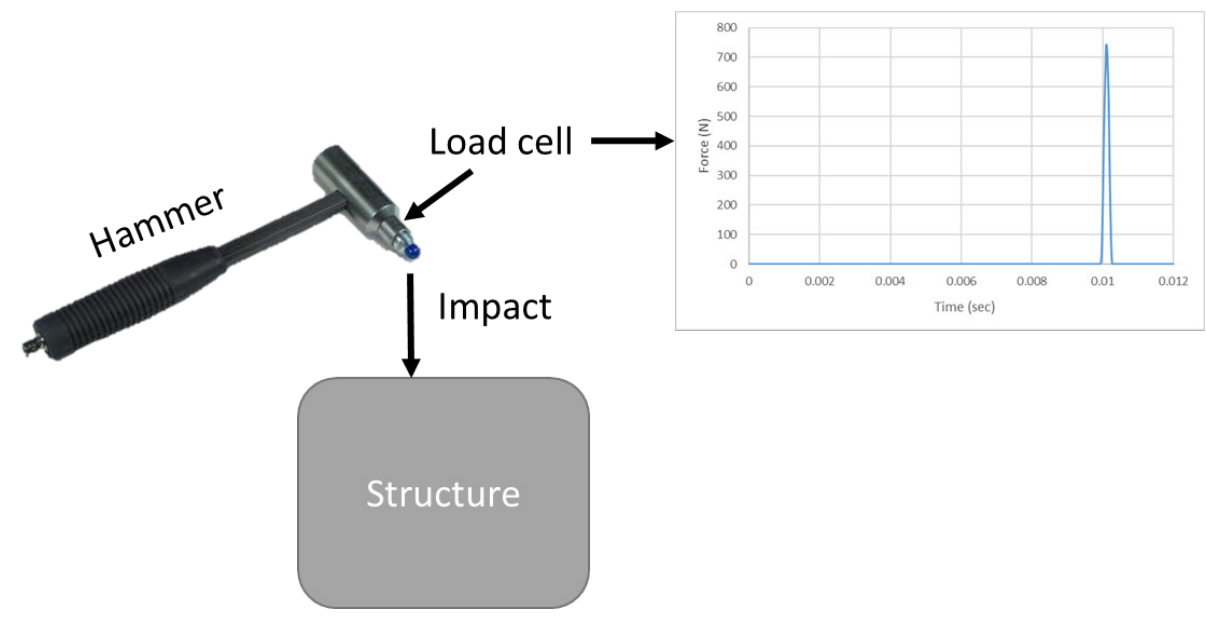

Figure 2.7 Schematic of impact source production in the laboratory.

\subsubsection{Pyroshock}

Pyroshock is another source that could be experienced by a structure, and is related to the impact source. Pyroshock is defined as a high-amplitude, short-duration, high-frequency 
event that can cause damage to small delicate components, such as electronics. This type of event is common in live ordnance detonation, drop impacts or between stages of rocket flight [10]. Pyroshock is difficult to reproduce in the lab, but it is often necessary to perform qualification tests relating to pyroshock due to its damage potential.

Pyroshock is typically reproduced in the lab using a resonant plate in a single axis. However, oftentimes, a pyroshock event is intertwined with some random excitation and it is desired to use a shaker table to include the entirety of the field source.

\subsubsection{Field Data Replication}

Field data replication is the act of reproducing a structure's field response with laboratory equipment, also known as waveform replication. Field data replication is done by playing a collected data set in real-time through a controller that automatically compensates for the shaker and fixture response. It is performed in a closed loop system, where we specify the control location and signal to replicate. The goal of this testing method is to be able to reproduce the same energy a component sees in the field, in the laboratory. These types of tests are normally conducted by gathering some field data and using it with waveform replication to create the lab test source.

\subsubsection{Paths, $[H]$}

The path in TPA is described as the structure or medium that the source is passed through to the receiver. In most shock and vibration problems, it is important to understand the path transfer functions and overall dynamics to be able to predict the response at the receiver. However, in order to do this, the path is often simplified to a lumped parameter model (LPM).

Lumped parameter models (LPMs) are commonly used in vibration problems to describe complex physical systems. All systems from an automobile to the human body can be described using a LPM with masses, springs, and dampers being the basic building blocks 
for vibration problems. LPMs are mathematically described using ordinary differential equations (ODEs). Each individual DOF present in a model can be described using an ODE called an equation of motion (EOM) derived from Newton's second law, developed from the free-body-diagram (FBD) of that particular DOF.

\subsubsection{SDOF Systems}

Single DOF systems are the simplest of LPMs, and are the basis for all vibration theory and analysis. SDOFs can be used to describe a very simple system or a continuous system. A continuous system is defined as a system that has physical parameters (e.g. mass, stiffness) that cannot be assumed isolated, meaning a continuous system has infinitely many DOFs [11]. Continuous system notation is often used in mechanics of materials, however when solving vibration problems, we generally discretize the system of interest to understand the inner dynamics of it. Therefore, most of the work presented in this thesis will be dealing with discrete systems.

Figure 2.8 shows a fixed SDOF mass-spring-damper model where the motion of this system as a result of the external force $f$, is described in the time domain by Equation 2.2.2.

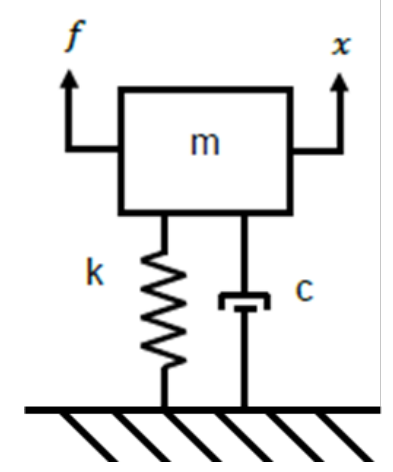

Figure 2.8 Single DOF model that is used as the basis to developing equations describing vibration problems.

$$
m \ddot{x}(t)+c \dot{x}(t)+k x(t)=f(t)
$$


Equation 2.2.2 is a linear time-invariant, second-order differential equation, using mass $(m)$, stiffness $(k)$, and damping $(c)$ parameters to describe the motion of a system, along with the dependent variable of displacement $(x)$, and the independent time $(t)$, where $f$ is the external force acting on the system. This equation and all systems can be described in the time, LaPlace, and frequency domains. For the purpose of this work, the focus will be on the time domain, as see in Equation 2.2.2, and the frequency domain described by Equation 2.2.3.

$$
-\omega^{2} m X(\omega)+j \omega c X(\omega)+k X(\omega)=F(\omega)
$$

Where $\omega$ is the frequency vector of interest, and $X(\omega)$ describes the displacement of the system over that frequency range as a result of the force acting on the system, $F(\omega)$.

When describing motion, several equations are derived for a SDOF system using mass, damping, and stiffness parameters of the system as seen in Equations 2.2.4 through 2.2.7.

Natural frequency:

$$
\omega_{n}=\sqrt{\frac{k}{m}}
$$

Damping ratio:

$$
\zeta=\frac{c}{2 m \omega_{n}}
$$

Damping factor:

$$
\sigma=\zeta \omega_{n}
$$

$$
\text { Damped natural frequency: } \quad \omega_{d}=\omega_{n} \sqrt{1-\zeta^{2}}
$$

\subsubsection{MDOF Systems}

Multiple DOF systems can be thought of as many SDOF systems attached to one another in a manner that represents the more complicated system. Figure 2.9 shows how a building can be modeled as a MDOF system using lumped parameter modeling where F represents the external force acting on the building. 


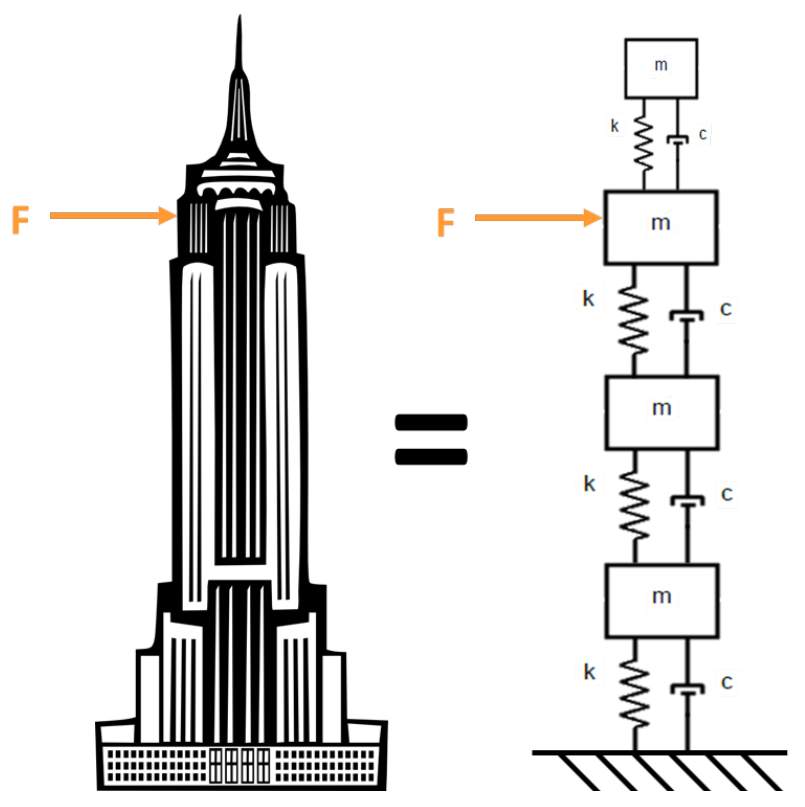

Figure 2.9 Multiple degree of freedom representation of a skyscraper with an external force.

MDOF equations of motion (EOMs) are typically organized in a matrix form as seen in Equations 2.2.8 and 2.2.9, where the columns and rows are equivalent to the number of DOFs present in the system $(n)$.

$$
\begin{gathered}
{[M] \ddot{\bar{x}}+[C] \dot{\bar{x}}+[K] \bar{x}=\bar{f}} \\
\left(-\omega^{2}[M]+j \omega[C]+[K]\right) \bar{X}=\bar{F} \\
\text { Where, } \quad \bar{f}=\left\{\begin{array}{c}
f_{1} \\
\vdots \\
f_{n}
\end{array}\right\} \text { and } \bar{x}=\left\{\begin{array}{c}
x_{1} \\
\vdots \\
x_{n}
\end{array}\right\}
\end{gathered}
$$

Normally, we assume proportional damping which is described as the relationship in Equation 2.2.10. The $a$ and $b$ coefficients are constants that show that damping is a linear combination of the mass and stiffness matrices.

$$
C=a K+b M
$$


These equations will be used throughout this research as a base for most calculations, as the systems we are interested in are MDOF.

\subsubsection{Frequency Response Functions}

Equation 2.2.3 can be rearranged to produce the equation for the frequency response function (FRF) of the SDOF system (Equation 2.2.11).

$$
\frac{X(\omega)}{F(\omega)}=\frac{1}{\left(k-\omega^{2} m+j \omega c\right)}
$$

This equation is graphically realized in Figure 2.10, where the natural frequency, and stiffness, damping, and mass controlled regions are identified for the SDOF system.

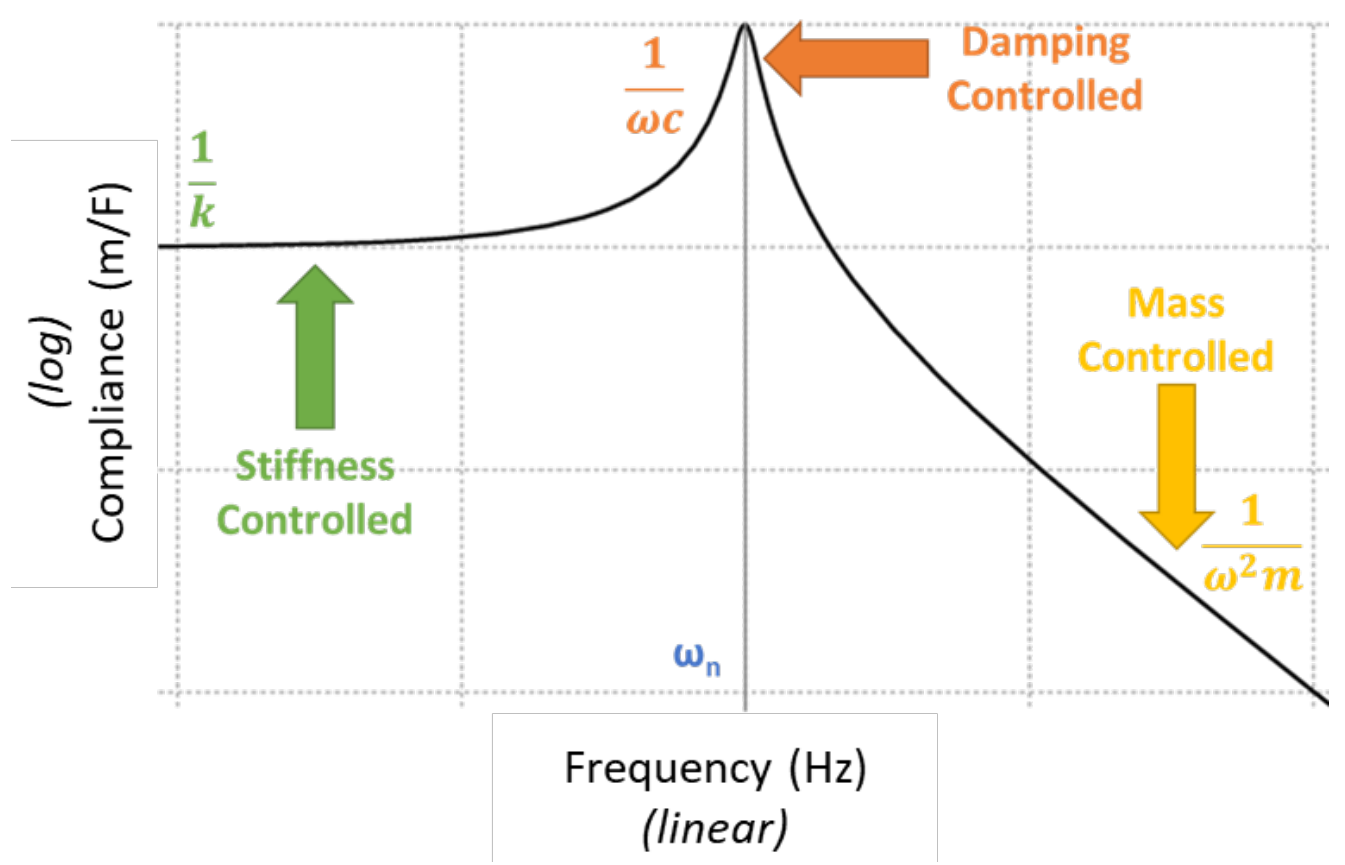

Figure 2.10 Graphical representation of the Frequency Response Function (Compliance) depicting the mass, damping, and stiffness controlled regions.

Equation 2.2.11 from is expressed in terms of the dependent variable being displacement $(X)$, and the independent variable $(F)$. This equation can be represented in different forms 
by deriving the equation to result in the dependent variable being velocity $(\dot{X})$, or acceleration $(\ddot{X})$. The equation can also be expressed as force $(F)$ over response, or response over force.

Table 2.1 Forms of the frequency response function (FRF) equation.

\begin{tabular}{lcll}
\hline Compliance: & $\boldsymbol{X} / \boldsymbol{F}$ & Dynamic Stiffness: & $\boldsymbol{F} / \boldsymbol{X}$ \\
\hline Mobility: & $\dot{X} / F$ & Impedance: & $F / \dot{X}$ \\
\hline Accelerance: & $\ddot{X} / F$ & Dynamic Mass: & $F / \ddot{X}$ \\
\hline
\end{tabular}

Table 2.1 displays the six different FRF equations. To achieve each form of the FRF equation, derivation in the frequency domain is necessary. Mobility is calculated by multiplying compliance by $j \omega$, and accelerance is compliance multiplied by $-\omega^{2}$.

Each of the six FRF equations are used for different applications. In this work, the accelerance measured at the locations at which the $\mathrm{COI}$ attaches to the box is used to design a fixture to produce the same FRFs at these locations. Although acceleration was measured, it can be integrated and inverted if impedance is desired, but the concept is the same with any of the FRF equations. The FRF describes the relationship between the input force and the receiver.

The FRF or characteristic equation of a structure is able to identify the mode shapes and natural frequencies of the structure through the eigensolution. The mode shapes are identified as the eigenvectors and the natural frequencies the eigenvalues. The eigensolution uncouples the relationship between the mass, stiffness, and damping through the eigenvalue problem. Assuming damping is proportional to the mass or stiffness matrix, the eigensolution can be written as Equation 2.2.12.

$$
[[K]-\lambda[M]] \bar{x}=\overline{0}
$$

Equation 2.2.13 relates the modal DOFs, $\bar{q}$, to the physical DOFs with the eigenvector matrix as, $[U]$. 


$$
\bar{X}(\omega)=[U] \bar{q}(\omega)
$$

Additionally, Equation 2.2.14 relates the frequency, $\omega$, to the eigenvalues, $\lambda$.

$$
\lambda=\omega^{2}
$$

From the above equations we are able to define the mode shapes and natural frequencies of any given system.

\subsection{Substructuring}

Dynamic substructuring is used to evaluate the dynamics of structures that cannot be evaluated as a whole [12]. Not only does dynamic substructuring allow us to analyze systems that are too large to evaluate as a whole, it also allows us to identify local dynamic behavior, combining model and experimental components, and combine structures from different project groups [12]. Dynamic substructuring is rooted in the foundation of "domain decomposition," [12] where solving a problem becomes easier when you break the problem into parts, and analyze each part individually.

Figure 2.11 shows a schematic of the substructuring theory combined with TPA theory. Consider TPA theory: the source input is located at point 1, the path (defined by the dashed line) can be broken into the transfer functions through subsystem $\mathrm{A}$, the interface, and subsystem B. Finally, we recognize point 3 as the receiver location. 


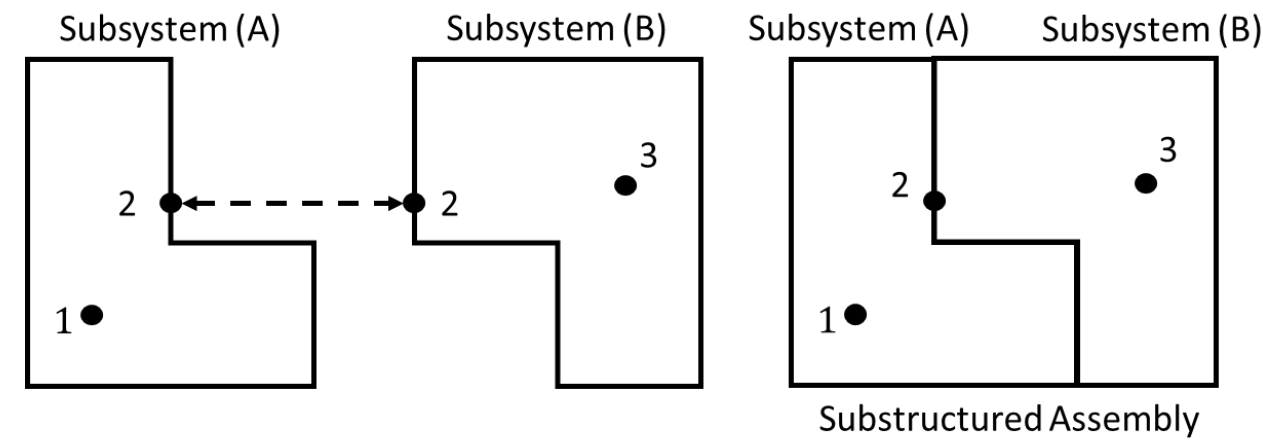

Figure 2.11 Schematic of two subsystems before and after substructuring, where point 2 represents the attachment DOFs and interface forces.

Each subsystem has some external and interface DOFs. The interface DOFs are the attachment locations. These points must have the same geometry between subsystems. The internal DOFs and attachments are denoted in Figure 2.11 by point 2 on subsystems $A$ and $B$. The external DOFs are the input or response locations on the full assembly, or any other points of interest $[10,13]$, in this case represented by the input at point 1 and response at point 3. In addition, there are two conditions that must be fulfilled for the subsystems to be coupled $[12,13]$ :

1. Coordinate compatibility condition: interface DOFs have equivalent displacement with a rigid (infinitely stiff) connection.

$$
2_{I}^{A}=2_{I}^{B}
$$

2. Force equilibrium condition: interface forces between DOFs are equal and opposite.

$$
2_{F}^{A}=-2_{F}^{B}
$$

When performing substructuring calculations, in addition to the conditions presented above, some assumptions must be made. These assumptions include: the interface DOFs being lumped to a single point and the FRF matrices produced being fully populated, meaning all DOFs present at the interface must be calculated [13] 
Dynamic substructuring can be defined in three domains: physical, modal, and frequency. Frequency and modal substructuring are results of some transformation from the physical domain as seen in Figure 2.12. In all three domains, the uncoupled subassemblies are individually defined by an equation of motion (EOM). Each domain characterizes the structure differently. In the physical domain, the structure is characterized by its mass, stiffness, and damping properties. In the modal domain, the structure is characterized by a combination of modal responses. Finally, in the frequency domain, the structure is characterized by its FRFs [12].



Figure 2.12 Dynamic substructuring domains and the conversions necessary between the three.

\subsubsection{Physical Domain}

Physical substructuring is performed by coupling and modifying the mass, stiffness, and damping matrices of the subsystems. Equation 2.2.3 represents EOMs in the physical domain for the subassemblies from Figure 2.11, where M, C, and $\mathrm{K}$ are the individual subassemblies mass, damping, and stiffness matrices, respectively. The superscript $i$ is either subassembly $A$ or $B$, the motion of the model is represented by $\bar{X}$, and $\bar{F}$ is the force acting on the structure.

$$
\left[M^{i}\right] \ddot{\bar{X}}^{i}(\omega)+\left[C^{i}\right] \dot{\bar{X}}^{i}(\omega)+\left[K^{i}\right] \bar{X}^{i}(\omega)=\overline{0}
$$

The individual subassemblies are coupled through forming the $\mathrm{M}, \mathrm{C}$, and $\mathrm{K}$ matrices into block-diagonal form and introducing the interface forces, $\bar{g}$, as seen in Equation 2.3.4. 


$$
M \ddot{\bar{X}}+C \dot{\bar{X}}+K \bar{X}=\bar{F}+\bar{G}
$$

\subsubsection{Frequency Domain}

Frequency based substructuring (FBS) calculates the fully-assembled systems dynamics using the FRFs from the individual subsystems present in the structure. FRF testing was performed on each individual subsystem and then FBS was used to calculate the full system dynamics. Recall the equation for the compliance FRF as seen in Equation 2.3.5, where the subscript $p$ is the point location on the system, and superscript $i$ is the subassembly.

$$
H_{p}^{i}=\frac{\bar{X}}{\bar{F}}=\left[-\omega^{2}\left[M^{i}\right]+j \omega\left[C^{i}\right]+\left[K^{i}\right]\right]^{-1}
$$

When we perform frequency based substructuring the FRFs from each subassembly are coupled to obtain the fully assembled system FRFs. The method used in this thesis is a TPA based method. The FRFs from the attachments at subassembly $A$ are coupled with the FRFs at the attachments from subassembly $B$ using Equation 2.3.6 and the notation of Figure 2.11.

$$
\bar{X}_{3}=H_{3 F}^{B}\left[H_{2 I}^{B}+H_{2 I}^{A}\right]^{-1} H_{2 F}^{A} \bar{F}
$$

The kernel, $\left[H_{2 I}^{B}+H_{2 I}^{A}\right]$, is where the FRF coupling occurs and is multiplied by the FRFs of subsystem $B, H_{3 F}^{B}$, and the free response of subsystem $A, H_{2 F}^{A} \bar{F}=\bar{X}_{A}^{2}$, to obtain the substructured system response, $\bar{X}_{3}$.

In one study, FBS was used as a means of altering the input excitation to eliminate the dynamic interaction of the vibration test fixture. This was necessary when the test fixture dynamically couples with the COI [14]. Using a steel frame and an aluminum plate the researchers attached the plate at multiple locations on the frame to perform this analysis. They collected experimental drive, and cross-point FRFs at the attachment points of the structures and performed FBS to determine the full-assembly dynamics [14]. 


\subsubsection{Modal Domain}

Through performing an eigenvalue analysis, we can bring the equations from the physical to the modal domain to perform substructuring. Modal substructuring is completed in the modal domain by coupling mode shapes. Equation 2.3.7 is the final EOM in the modal domain where $\widetilde{M}, \tilde{C}$, and $\widetilde{K}$ are the modal mass, damping, and stiffness matrices, respectively.

$$
[\widetilde{M}] \ddot{\bar{q}}(\omega)+[\tilde{C}] \dot{\bar{q}}(\omega)+[\widetilde{K}] \bar{q}(\omega)=[U]^{T} \bar{F}(\omega)
$$

The mass and stiffness matrices of the subassemblies are brought to the modal domain and coupled physically through adding stiffness to the interface DOFs. The eigenvalue problem is then solved for the coupled system, producing new natural frequencies and mapping mode shapes which are used to transform the uncoupled mode shapes to the coupled/substructured mode shapes.

With respect to boundary condition replication, some studies have been done using modal based substructuring. One study uses modal substructuring to quantify the magnitude of discrepancy between field and laboratory testing [15]. Understanding the discrepancies can assist the engineer in making design decisions for the laboratory fixture to properly reproduce field BCs. The study discusses the substructuring transformation and how it decouples the system into fixed-base modes of the COI and the free modes of the nextstructure. This is valuable because we are then allowed to illustrate the dynamic response of the $\mathrm{COI}$ in terms of fixed motion, so we can capture the damaging energy the $\mathrm{COI}$ is exposed to without the non-damaging motion [15].

\subsection{BARC Studies}

The original intent of the structure was for it to be used to design an environmental shock and vibration test fixture for a component. The research team developing the hardware accomplished the following in their design of the BARC: 
1. The model includes a full assembly, allowing for creation of a field environment and provide "truth" data.

2. The model is simple and the component is easily detachable for individual testing.

3. The assembly is low cost.

4. The component is small enough to test on a small shaker system or shock machine.

5. The component response is non-trivial, so single-axis inputs result in multi-axis motion.

6. The component and assembly interact dynamically.

7. The over and undertesting problem manifests when using a rigid fixture approach.

Since its introduction, the BARC structure has continued to gain popularity in the dynamic environmental testing community. Many studies have been conducted using the structure, some with the intent of understanding its overall dynamics, and some with the boundary condition challenge goal in mind.

\subsubsection{Modal Analysis}

Several collaborators have completed modal tests of the BARC structure. Figure 2.13 shows the geometry created by 4 of those entities compared in a paper presented at IMAC by Sandia National Laboratories [16]. Sandia National Laboratories introduced 5 different sets of geometry, but only 4 were used in its analysis, those 4 are shown here.
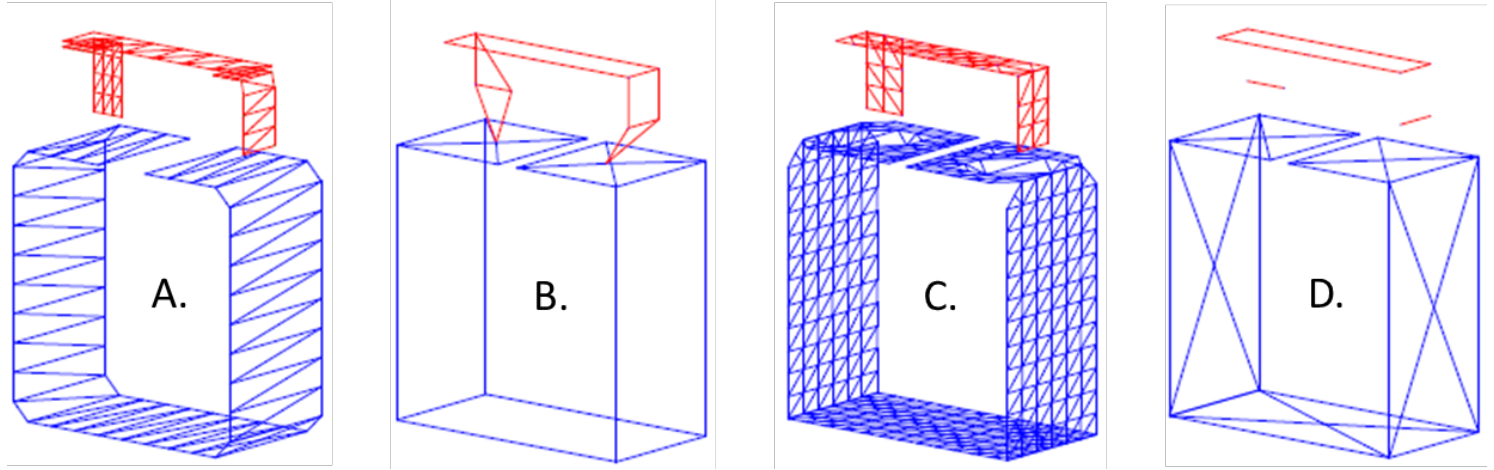

Figure 2.13 Geometry used for 4 different datasets during modal testing of the BARC from 4 collaborators on the Boundary Condition Challenge Problem [16]. 
In addition to each set of geometry being different, the modal tests performed using each configuration were different. However, we are not concerned with the differences in tests, just that they all produce similar results with respect to the BARC structure's dynamic motion.

In order to accurately compare the different tests, mapping between the modes extracted in each test must be completed, which is traditionally done by comparing mode shapes of the different tests [16]. Since the geometry is different for each test, comparing shapes is difficult, therefore a finite element model was used to expand each set of mode shapes to allow for a consistent set of measurement DOFs [16]. Figure 2.14 shows the FEM that was used for the expansion procedure created by Sandia National Laboratories for other BARC work. All modes within the bandwidth of each of the tests described were used in the expansion procedure, including rigid body modes [16].

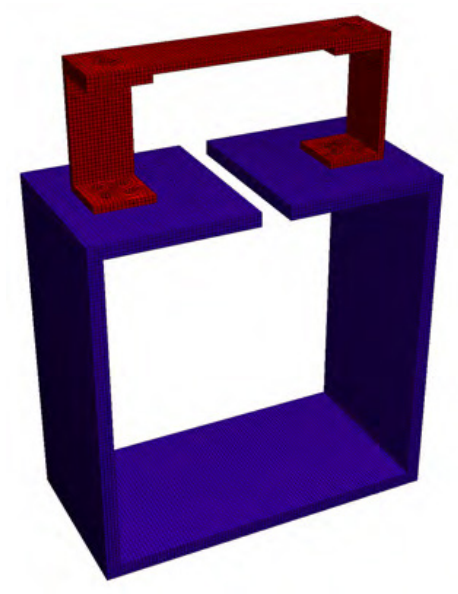

Figure 2.14 FEM used for shape expansion to compare 4 different modal tests performed on the BARC structure [16].

As a result of the expansion, animated modes were extracted from the FEM. Figure 2.15 depicts the FEM expansion from the test geometry for the first elastic mode [16]. There are some visible differences in the 4 tests, this is a result of how the tests were performed, whether with roving impact, shaker excitation, and/or roving accelerometer. 




Figure 2.15 FEM expansion from 4 different test geometries shown in Figure 2.13 for the first elastic mode [16].

The goal behind looking at the motion of the BARC structure is to design a fixture with a fixed base to attach to test equipment that will produce similar motion at the box/component attachment locations. We see that for this first non-rigid mode, the two feet of the COI move in the vertical direction out of phase with one another. This reinforces the idea that mounting the $\mathrm{COI}$ to a rigid fixture would not produce the proper response across the COI.

\subsubsection{Fixture Optimization FEA}

Using parameter or topology optimization as a method of designing a dynamic test fixture has recently been explored using the BARC and other hardware. Using TPA and FBS concepts and optimization models, the finite element models of the BARC and a two-beam structure were analyzed. In all cases, a common rigid test fixture was analyzed alongside the optimized dynamic fixture to rate its success in improving the similarity to the field response on the component.

In one study, the laboratory source for the rigid fixture was derived using enforced motion where the motion at the base of the next-assembly was measured and used to force the lab 
fixture to match. The topology optimized fixture setup was tested using a forcing function that was computed by multiplying the pseudo inverse of the FRFs of the lab configuration by the target response of the component [2]. The results of this study show that the topology optimized test fixture did not perfectly reproduce the stress seen in the field, but had some advantage over the rigid test fixture. The optimized fixture resulted in slightly less over testing at points on the structure, but didn't exactly replicate the response seen in the field [2]. This work showed that optimization is a possible method for designing dynamic test fixtures, however there is a need to limit the parameters to be optimized. It showed that even though it only solved to local minima in can have major improvements in the resulting stresses relative to a common fixture design [2].

Another study, explored using an optimizable parameterized model to design a dynamic test fixture for the BARC hardware. The goal of this model is to produce a dynamic test fixture that matches the impedance of the next-level assembly to allow for the component to experience similar stresses as it sees in its field environment. It was shown that using FRFs as optimization functions often result in local minima that cause the optimization to have difficulty converging on a meaningful answer. However, most objective functions used in this optimization come from FRF objective functions [17]. This work examined the objective functions capability when varying one and two design parameters of a LPM. The results of this showed that the number of local minima would increase with the number of design parameters included in the optimization [17]. Without restricting the design space, optimizing based on three or more parameters becomes too computationally difficult. This work performs a case study of impedance matching using the optimization tool on the BARC hardware. The BARC is parameterized and nodes are selected on the component for the objective function [17]. Essentially, they model versions of the Box by constraining parameters using FEA with the intent of producing similar attachment impedance. Since the parameters are constrained the new fixture design is unable to converge to the next-structure, but the optimization is successful in producing a solution that represents the dynamics over a conventional fixture [17]. 
Another study used finite element analysis to compare the BARC in different configurations to a "truth" data set. The study was performed using a single-axis excitation and a 6-DOF excitation. The configurations included a rigid fixture where the component was mounted to a rigid plate the same dimensions as the top of the box, and a " $\mathrm{N}+1$ " fixture which included a 1" and 3" addition to either end of the plate [18]. The results from these tests showed that the single-axis excitation did not accurately produce the acceleration or strain seen on the component in the field with or without a fixture. However, this could be a result of the model not including the cut between the attachment points of the component. The truth data was created using many inputs in varying directions, and the 6-DOF excitation shows a closer representation of the truth data than the single axis as a result of this [18].

\subsubsection{Multi-Axis Testing}

Some multi-axis testing has been performed on the BARC structure with the intent of using it to better mimic real world conditions. One of these tests mounted the component to topology optimized AM brackets attached to a vibration cube and introduced a multi-axis excitation [19]. Another fixed the component directly to the vibration cube and introduced a multi-axis excitation. Both found that without proper representation of field BCs a multiaxis test does not provide any more representative response as a single-axis test [19].

Another study treated the two attachments of the component of the BARC as two 6-DOF shaker control systems. The goal of this analysis was to remove the fixture interaction in general and replace it with motion defined by the field environment presented unto the component through the shaker excitation at each attachment. The results showed that this method could be feasible if the excitation is representative of the forces produced from the next-structure in the field environment [20]. 


\section{Theory Applied to BARC}

This chapter describes the theory behind the work presented in this research. It goes over when it is necessary to develop a dynamic test fixture. It also discusses the use offrequency based substructuring and attachment impedance matching as it applies to the new fixture design process and the BARC structure, specifically.

\subsection{BARC Frequency Based Substructuring}

Shown in Figure 3.1, we broke the BARC into its two components: Box and COI. This allowed us to analyze the attachment DOFs labeled $A_{1}$ and $A_{2}$. The variable, $X$, indicates the response at the attachment location. The superscripts $C$ and $B$ specify which side of the attachment we are analyzing, COI or Box, respectively, while the subscripts 1 and 2 indicate the attachment location.



Figure 3.1 Schematic of the BARC broken into two parts for attachment DOF analysis $\left(A_{1}\right.$ and $\left.A_{2}\right)$, showing external forces $\left(\bar{F}_{B}\right)$ and target data $\left(\bar{X}_{T}\right)$. 
Also shown are the external forces $\left(\bar{F}_{B}\right)$ acting on the structure and the target data $\left(\bar{X}_{T}\right)$ across the top of the COI, or the response of the assembly. This target data is what we tried to replicate with a next-level assembly on the shaker table. It could be acceleration, stress, strain, etc. With the same motion at the attachments, the motion across the top of the COI must be the same with the proper excitation profile.

There are some assumptions that we made when doing this analysis:

1. The attachments are lumped to a single point.

2. We are only considering translational DOFs (X, Y, and Z).

3. FRF matrices must be fully populated, $6 \times 6$ matrix.

Assumption 3 brings up the FBS math that is used to define the testing that was done, described by Equations 3.1.1 and 3.1.2. As we know, it is necessary to have a fully populated FRF matrix to successfully perform FBS.

$$
\bar{X}_{T}=\left[H_{X A}^{C}\right]\left[\left[H_{A A}^{C}\right]+\left[H_{A A}^{B}\right]\right]^{-1}\left[H_{A F}^{B}\right] \bar{F}_{B}
$$

Each set of brackets represents a different FRF matrix needed to complete the FBS calculation. $\left[H_{X A}^{C}\right]$ is the FRF matrix from the inputs to the $\mathrm{COI}$ at the connection and output at the top of the component. While, $\left[H_{A A}^{C}\right]$ and $\left[H_{A A}^{B}\right]$ attachment FRFs on the component and box, respectively. These two FRF matrices make up the "kernel" of the FBS calculation. With a rigid fixture we are allowed to neglect the $\left[H_{A A}^{B}\right]$ matrix, as it does not produce additional dynamics to the assembly. However, in the case of the BARC structure $\left[H_{A A}^{B}\right]$ cannot be ignored since the $\mathrm{COI}$ is stiffer than the box in its field environment.

The free-response at the box attachment locations is defined by $\left[H_{A F}^{B}\right] \bar{F}_{B}$. This free response is obtained by gathering FRFs from the connection of the subassembly without the COI attached. The FRF matrix can be described in terms of displacement, velocity, or acceleration. When we multiply the FRF matrix by the force we get the dynamics at the connection locations. This means we do not need to know what the input force or dynamics 
are for the box, we only need to know the dynamics of the attachment locations without the COI.

$$
\bar{X}_{T}=\left[H_{X A}^{C}\right]\left[\left[H_{A A}^{C}\right]+\left[H_{A A}^{B}\right]\right]^{-1} \bar{X}_{A}^{B}
$$

Understanding the motion of the attachment DOFs, in this case we used accelerance, allowed us to design a fixture that could reproduce this similar motion at the attachment locations as the next-structure (box). Figure 3.2 shows a similar schematic to Figure 3.1, except this figure shows the fixture instead of the next-structure. The fixture is fixed to a shaker table where some forcing function is applied. This force is aimed to assist the fixture in reproducing the kernel of the FBS from Equation 3.1.1.

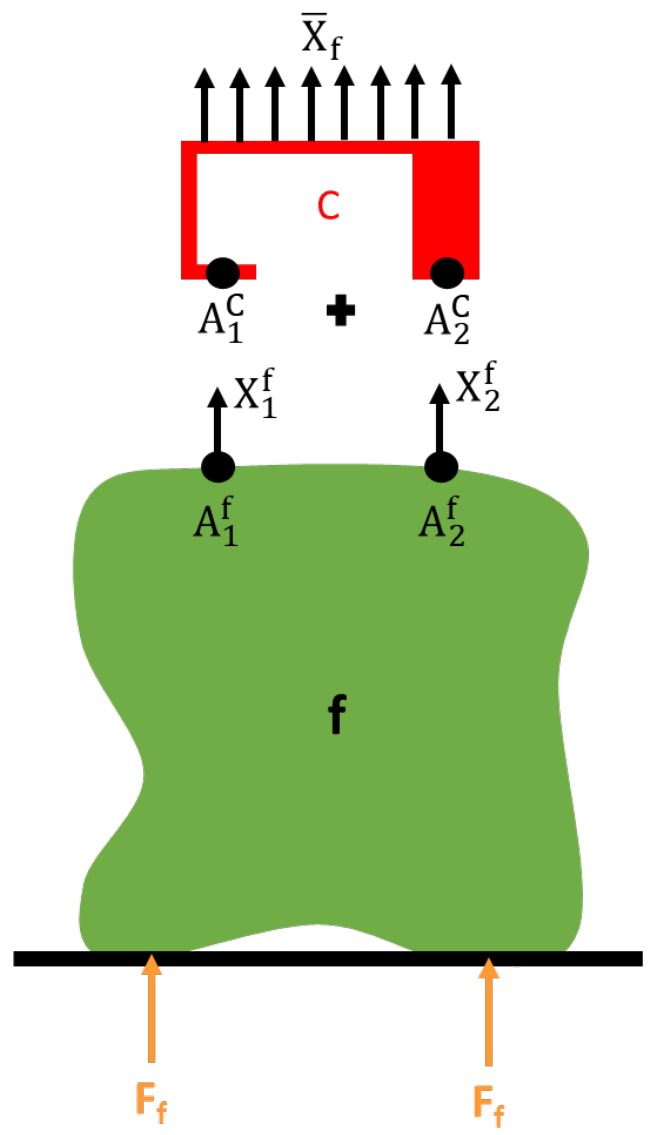

Figure 3.2 Schematic of the component and fixture for attachment DOF analysis $\left(A_{1}\right.$ and $\left.A_{2}\right)$, showing external forces $\left(\bar{F}_{f}\right)$ and fixture-component response $\left(\bar{X}_{f}\right)$. 
Equation 3.1.2 can be rewritten with respect to the fixture-component assembly (Equation 3.1.3) by replacing the box attachment FRF matrix with that of the fixture, $\left[H_{A A}^{f}\right]$. We also replace the free-response of the box with the fixture free response, $\bar{X}_{A}^{f}$.

$$
\bar{X}_{f}=\left[H_{X A}^{C}\right]\left[\left[H_{A A}^{C}\right]+\left[H_{A A}^{f}\right]\right]^{-1} \bar{X}_{A}^{f}
$$

We know that the free-response of the fixture is equivalent to the FRFs of the fixture as a result of the force multiplied by the force input, as seen in Equation 3.1.4.

$$
\bar{X}_{A}^{f}=\left[H_{A F}^{f}\right] \bar{F}_{f}
$$

We know that in order to achieve an equivalent response across the component in its field and laboratory settings, meaning $\bar{X}_{T}=\bar{X}_{f}$, then the following relationships must be true:

1.) $\quad\left[H_{A A}^{f}\right]=\left[H_{A A}^{B}\right]$

2.) $\quad \bar{X}_{A}^{f}=\bar{X}_{A}^{B}$

We can adjust both the force input and the attachment FRFs of the fixture to ensure relationship two is satisfied. We can satisfy relationship one by determining mass, stiffness, and damping parameters and the number of modes present in the next-structure from $\left[H_{A A}^{B}\right]$. Upon knowing this information, a lumped parameter model can be designed with an equal number of DOFs, and optimized mass, stiffness, and damping values.

\subsection{BARC Analysis}

In order to design a fixture to attach to a shaker table that produces similar attachment FRFs as the box of the BARC structure, we must first understand the dynamic characteristics of the box alone and the full assembly. When the BARC was originally introduced to the shock and vibration community in 2018 the initial response by the community was to perform dynamic testing on the structure. MTU was one of the first partners to perform a 
modal test of the BARC structure, alongside several other entities that received hardware in 2018. These various modal tests proved to provide a good starting point for determining a design for a fixture to produce similar dynamics as the BARC. The purpose of this section is not to compare the methods used it testing the BARC, but to develop an understanding of the dynamic motion of the structure.

\subsubsection{Attachment Dynamics Analysis}

With an idea of the dynamics of the BARC, we followed an attachment FRF approach. Accelerance was used in this analysis, the concept is the same: to minimize the differences in attachment FRFs between the next-level assembly and the laboratory test assembly.

\subsubsection{Testing}

The box and COI were both tested in a free-free BC to gain the attachment FRF matrices. To design the fixture, we focused on the response at points 1 and 2 on the box. The test setup for obtaining these FRFs from the box and COI can be seen in Figure 3.3 and Figure 3.4 , respectively.

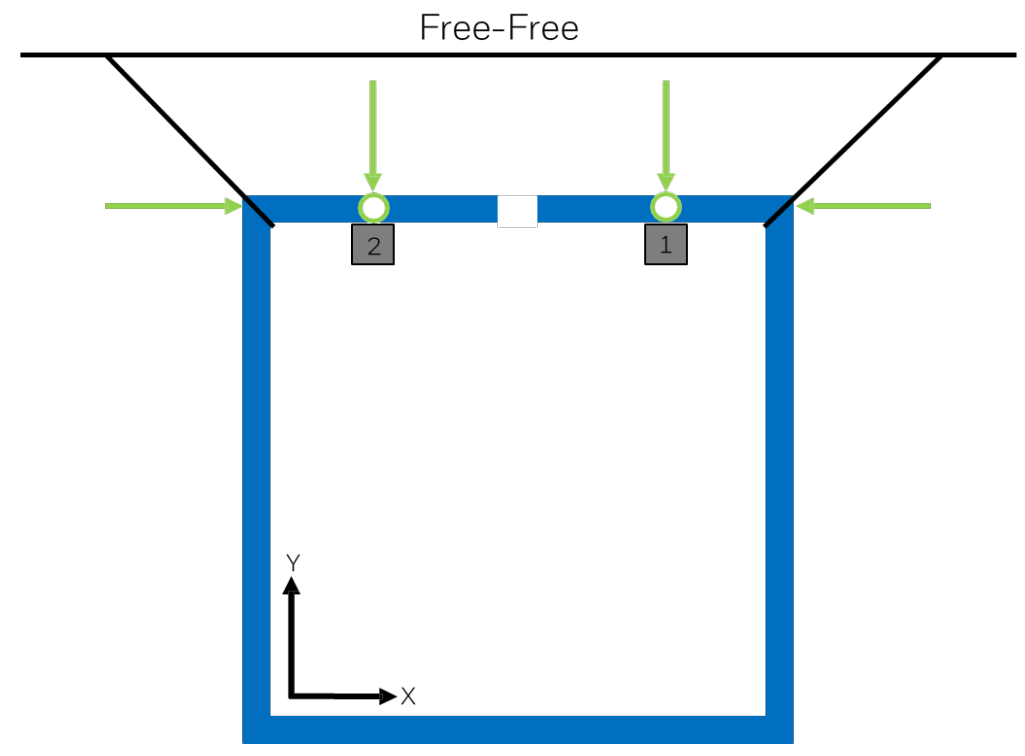

Figure 3.3 Box from BARC test set-up used to obtain FRF matrices from attachment locations. 


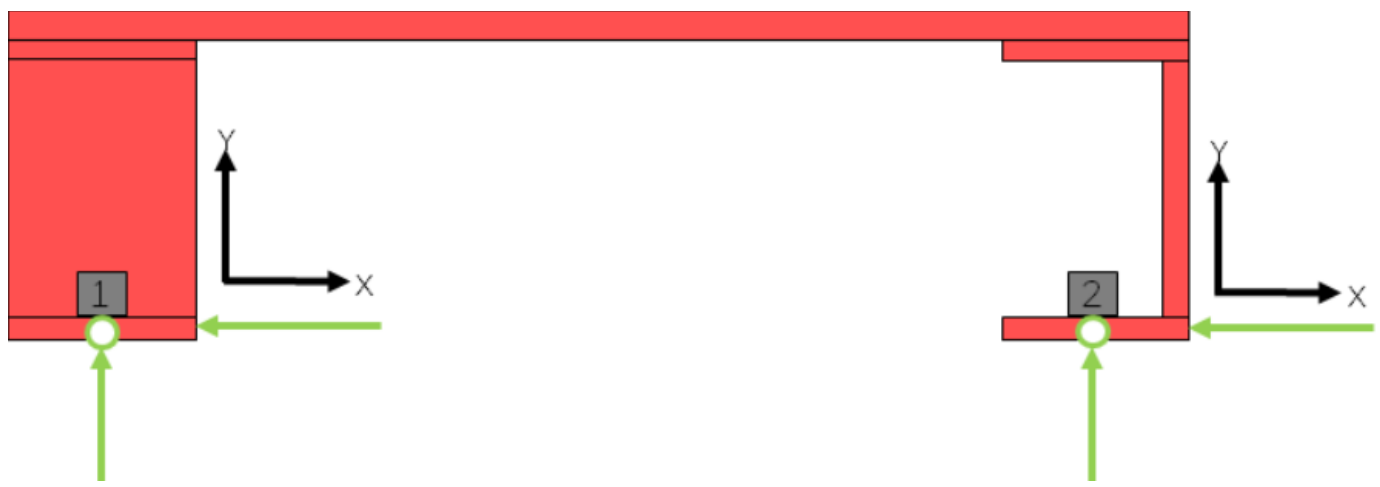

Figure 3.4 COI from BARC test set-up used to obtain FRF matrices from attachment locations.

Small tri-axial accelerometers were glued to the connection locations. An impact hammer was used to excite the structures by impacting in the $\mathrm{X}, \mathrm{Y}$, and $\mathrm{Z}$ directions near both connection locations. Responses from both accelerometers were recorded in the X, Y, and $\mathrm{Z}$ directions, resulting in a $6 \times 6$ matrix of FRFs. Figure 3.5 shows the accelerance matrix collected from the modal test performed on the box.

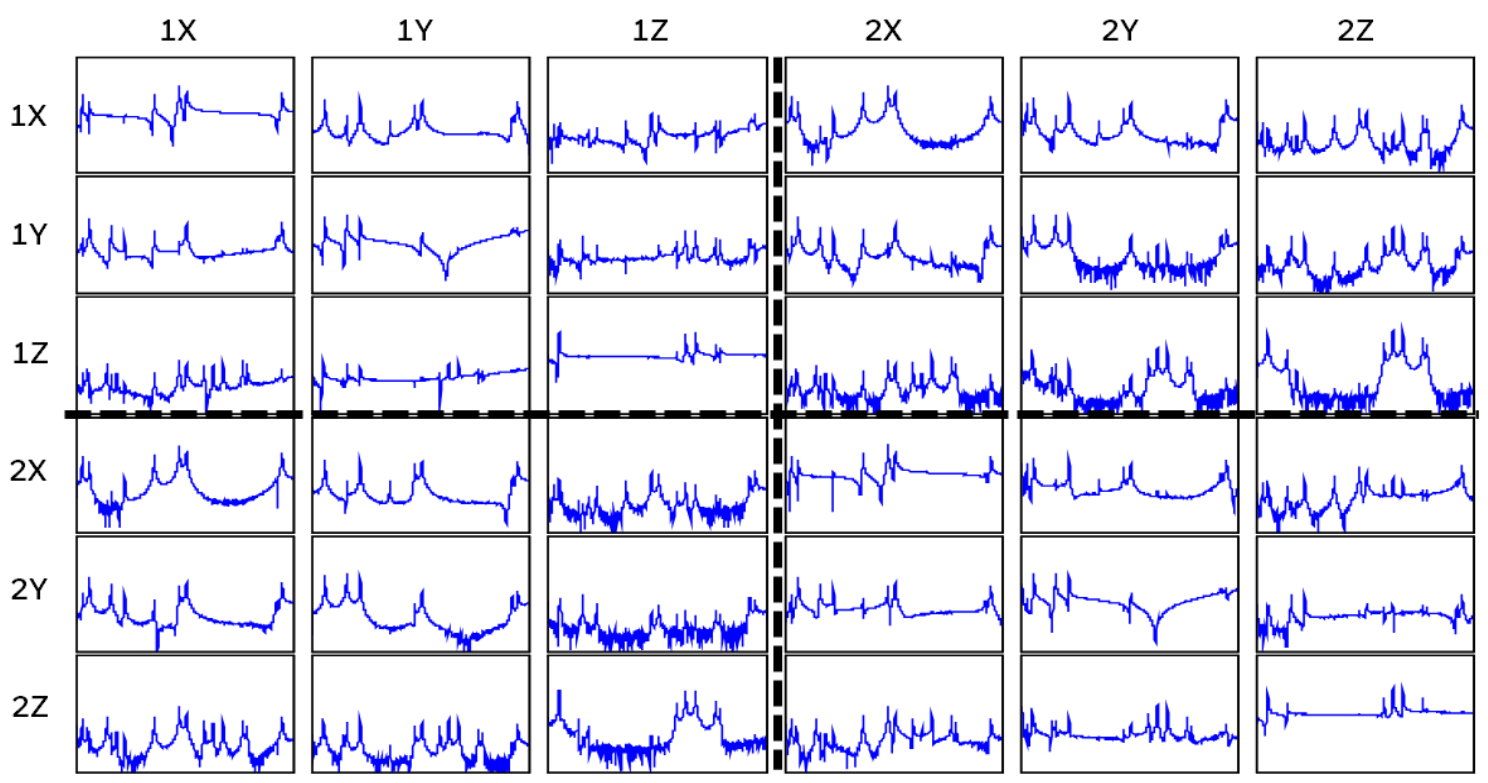

Figure 3.5 Accelerance Matrix from Free-Free Box Modal Test used to Determine Stiffness (Y-axis: Log 0.001-1000 g/N, X-axis: Linear 0-3200 Hz) 


\subsection{Component Analysis}

From a TPA standpoint we can determine whether or not a dynamic test fixture is necessary. The relative stiffness between the COI and path or next-structure determines whether or not a rigid fixture is sufficient. When the COI is softer than its next-structure, a rigid fixture is acceptable, as there is no relative dynamics between the attachment locations, which is most often the case. However, when the COI is stiffer than the nextstructure, it dominates the attachment motion and therefore, often produces relative motion between the attachments meaning that a dynamic fixture that allows for this motion is necessary to avoid over and/or undertesting the COI.

The FBS math described in the previous section can be used to better understand why the relative stiffness between the $\mathrm{COI}$ and the next-structure determines the necessary rigidity of the fixture. The kernel of the FBS equation is defined by adding the attachment FRFs of the two subassemblies, $\left[H_{A A}^{C}\right]+\left[H_{A A}^{f}\right]$. Since stiffness is defined as the inversion of amplitude at $0 \mathrm{~Hz}$, once the inversion of the kernel is complete, the stiffer set of FRFs dominates the equation. This means whichever subassembly is stiffer, will dominate the overall response of the full-assembly.

We performed some analysis using a MATLAB model with a LPM of the component and different stiffness next-structure LPMs to prove this theory. To quantify the stiffness of the component relative to the next structure we used Equation 3.3.1.

$$
\Delta=k_{\text {base }}-k_{\text {component }}
$$

Figure 3.6 shows the FRFs and SRS plots from three different models where the blue curve represents the attachment FRF from the next-structure, the green curve is the dynamic fixture, while the black curve is a rigid fixture. Descriptions of each plot are present following the figure. 

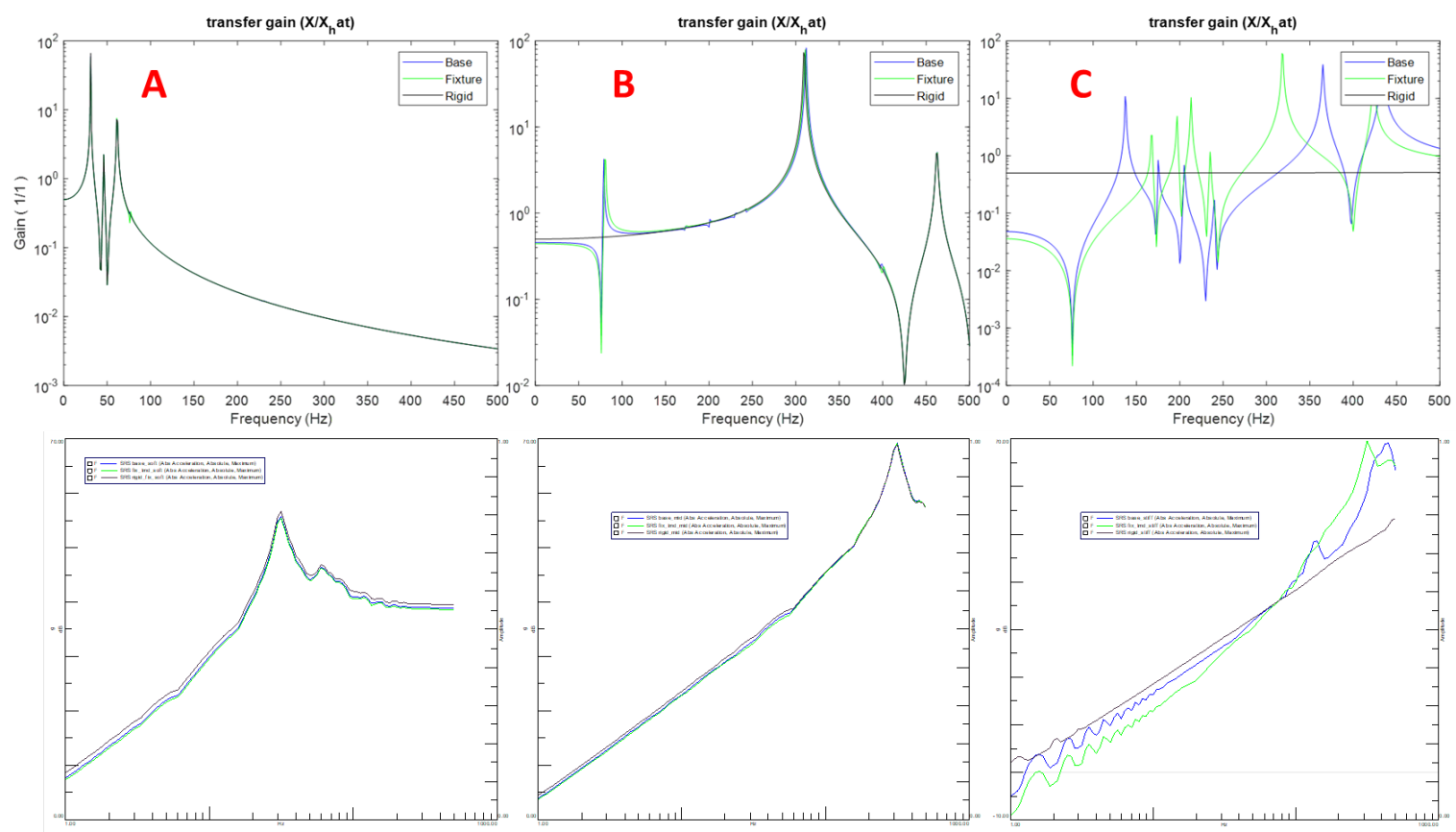

Figure 3.6 FRF and SRS plots comparing three different stiffness's for the next-structure to a rigid and a dynamic test fixture model.

A. Component is softer than next-structure $(\Delta>+10 \mathrm{~dB})$ : all modes of the next-structure are matched by the rigid fixture, so the response of the component is independent of fixture stiffness, and a dynamic fixture is unnecessary.

B. Component has similar stiffness to next-structure $(1 \mathrm{~dB}>\Delta>10 \mathrm{~dB})$ : some modes of the next-structure are missed by the rigid fixture, so the response is partially dependent on fixture stiffness, and additional dynamics may be necessary.

C. Component is Stiffer than next-structure $(\Delta>-10 \mathrm{~dB})$ : no modes of the next-structure are matched by the rigid fixture, so the response is sensitive to the fixture stiffness and dynamics, therefore a dynamic test fixture is necessary to accurately produce desired component response.

We then related this knowledge to the physical BARC. The comparison between the COI and the next-structures accelerance FRFs are seen in Figure 3.7. 


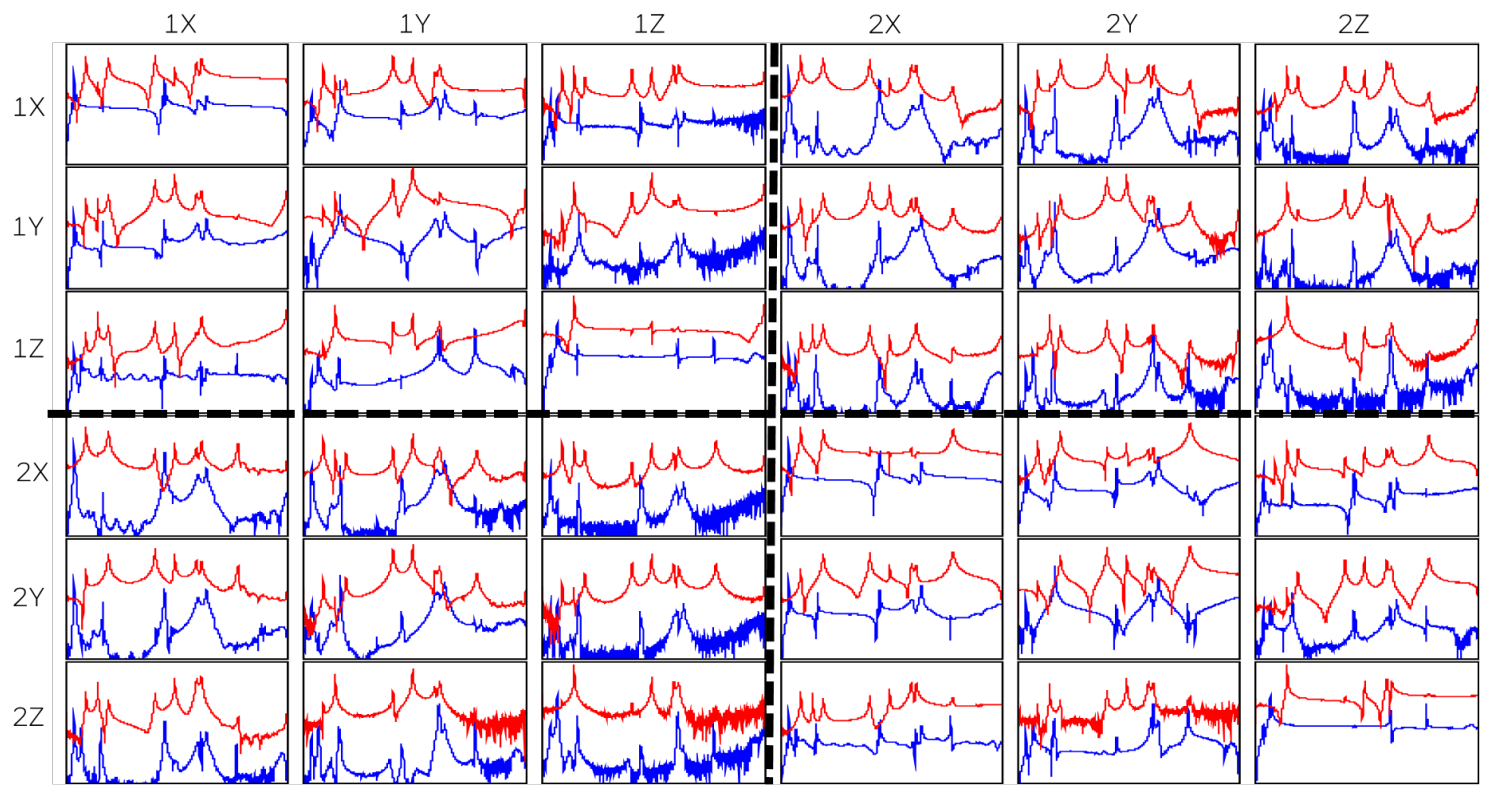

Figure 3.7 Comparison of component attachment accelerance (red) to next-structure (box, blue) attachment accelerance (Y-axis: Log 0.001-1000 g/N, X-axis: Linear 0-3200 Hz).

We can see that in three directions and between two attachments that overall the COI is stiffer than the next-structure, meaning that a dynamic test fixture is necessary, as predicted. 


\section{Initial Fixture Design}

This chapter discusses the original steps taken to tackle the fixture design problem using a lumped parameter model methodology, knowledge of the mode shapes of the BARC structure, attachment impedance, and stiffness of the box. It will start by covering the initial analysis performed on the structure and then how we used that knowledge to determine a potential fixture design. There will then be a discussion of the analytical and physical testing of the design and how the design compares to the BARC.

\subsection{Analytical Model}

The analytical model developed was designed from the free-free accelerance matrix of the box seen in Figure 3.5. The static stiffness of each FRF were extracted by inverting the accelerance value at $0 \mathrm{~Hz}$. In understanding the stiffness and the expected dynamics of the box, we were able to design a model to have similar response to the box.

\subsubsection{Design Development}

I organized the stiffness values into a table format with respect to the FRF they were extracted from to look at the directional stiffness of the box. These stiffness values are seen in Table 4.1 where they were gathered from the inverted compliance matrix. In the past, it was assumed that the cross FRFs were not of concern for fixture design. The cross FRFs represent the dynamics present between the two attachments and they are necessary to the FBS calculations and creating a dynamic test fixture. 
Table 4.1 Stiffness values in $\mathrm{kN} / \mathrm{m}$ from Box FRF matrix to be used to design the dynamic test fixture.

\begin{tabular}{|c|c|c|c|c|c|c|}
\hline & $\mathbf{1 X}$ & $\mathbf{1 Y}$ & $\mathbf{1 Z}$ & $\mathbf{2 X}$ & $\mathbf{2 Y}$ & $\mathbf{2 Z}$ \\
\hline $\mathbf{1 X}$ & $\mathbf{6 0}$ & 1200 & 1900 & $-\mathbf{7 3}$ & 175 & -1550 \\
\hline $\mathbf{1 Y}$ & 1200 & $\mathbf{8 8 0}$ & -2000 & 1140 & $-\mathbf{8 3 0}$ & 11100 \\
\hline $\mathbf{1 Z}$ & 1900 & -2000 & $\mathbf{2 9 0}$ & -2900 & 1840 & $\mathbf{- 3 0 0}$ \\
\hline $\mathbf{2 X}$ & $\mathbf{- 7 3}$ & 1140 & -2900 & $\mathbf{7 3}$ & -140 & 1990 \\
\hline $\mathbf{2 Y}$ & 175 & $-\mathbf{8 3 0}$ & 1840 & -140 & $\mathbf{2 3 0}$ & -1600 \\
\hline $\mathbf{2 Z}$ & -1550 & 11100 & $-\mathbf{3 0 0}$ & 1990 & -1600 & $\mathbf{3 3 0}$ \\
\hline
\end{tabular}

Table 4.1, and the analysis done in section 3.3, shows that they cannot be ignored. Attaching the COI to a rigid fixture, or a fixture that does not connect the attachments, would eliminate the cross FRFs. We called this theory the "individual spring method," meaning each foot of the component would be attached to an individual spring.

\subsubsection{Springs Method}

We tested this theory by performing impact tests, similar to those performed on the box and COI, on three sets of rubber pillars of durometers 40A (soft), 90A (hard), and 70D (extra hard), additively manufactured brackets produced by Honeywell (Figure 4.1), and a rigid fixture of common design. Equation 4.1.1 shows the mathematical theory behind this method, showing that it cannot produce the required fully populated FRF matrix, and therefore will not be able to reproduce the next-structure dynamics. Even from a multi-axis excitation, this method is not sufficient [19].

$$
\left[H_{A}^{D}\right]=\left[\begin{array}{cc}
\text { Stiffness } & 0 \\
0 & \text { Stiffness }
\end{array}\right] \neq\left[H_{A}^{B}\right]
$$




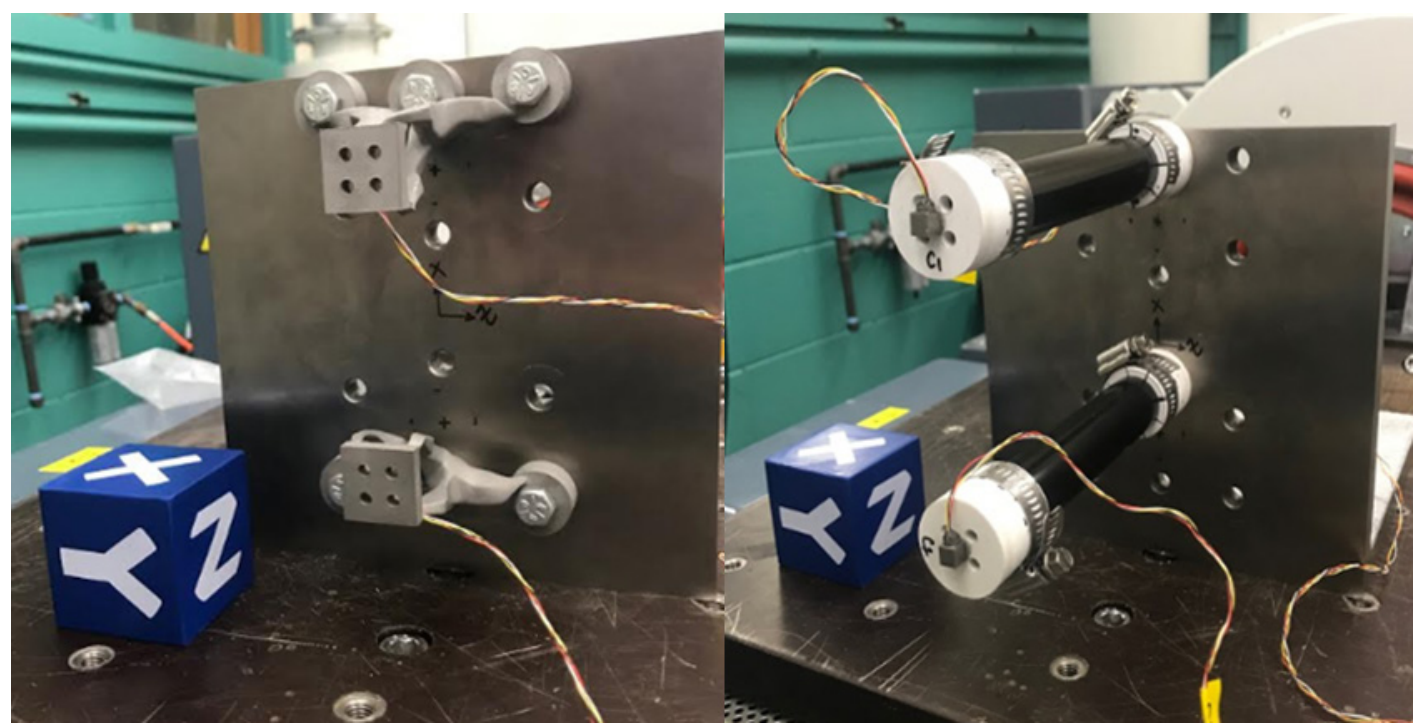

Figure 4.1 Physical Modal Test Performed on AM Parts and Rubber Pillars Mounted to Shaker as Rigid Base

The rubber pillars and AM part tests did produce cross FRFs because the L-bracket needed to mount them to the rigid shaker base had some low frequency dynamics. However, as expected from Equation 1, the cross FRFs did not match the box FRFs. The results can be seen in Figure 4.2 and Figure 4.3.



Figure 4.2 3-Hole Additively Manufactured Parts (Green) vs. Box (Blue) (Y-axis: Log 0.001-1000 g/N, X-axis: Linear 0-3200 Hz) 


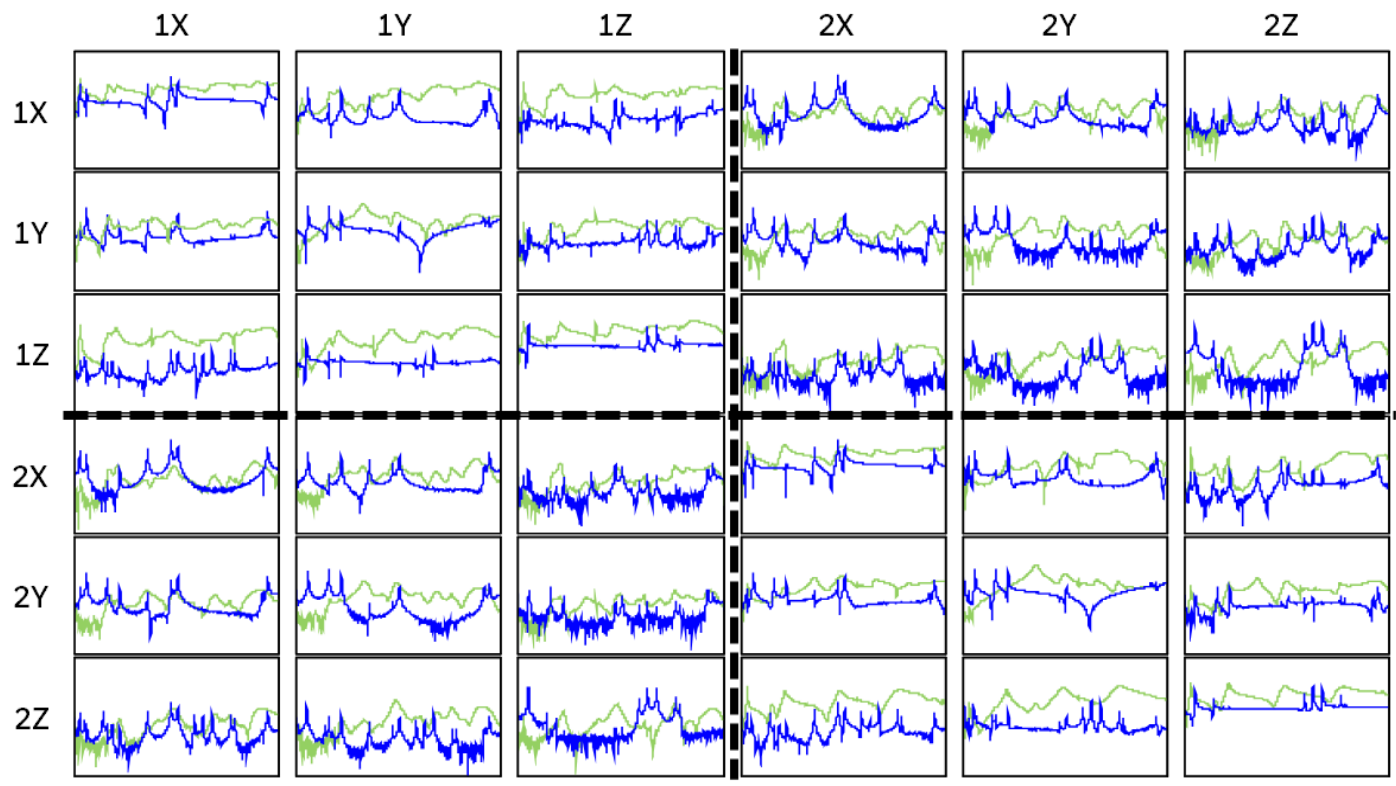

Figure 4.3 Rubber Bars (Green) vs. Box (Blue) (Y-axis: Log 0.0001-1000 g/N X-axis: Linear 0-3200 $\mathrm{Hz})$

In both cases, there are obvious peaks in accelerance, but they are not in the same location or amplitude as the box. The L-bracket caused more damping, while the stiffness of the assemblies seems to be similar to that of the box.

\subsubsection{Stiffness Analysis}

As expected, individual springs, as well as a rigid fixture, did not produce the necessary excitation in the cross FRFs. This proved that something connecting the attachments in a non-rigid manner was required. Figure 4.4 shows a LPM schematic of the most simplified version of what a dynamic test fixture must include in order to produce cross FRFs.

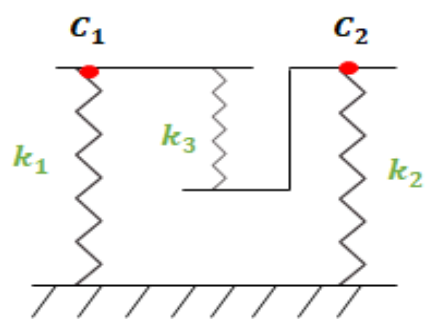

Figure 4.4 Simple schematic of dynamic test fixture design based on connecting spring concept, a connection between the attachment locations is necessary to produce a full FRF matrix. 
We used this concept in conjunction with the stiffness values collected from the box data, seen in Table 4.2, where we can see that the Y-direction was the stiffest at both attachment locations.

Table 4.2 Directional stiffness values collected from free-free Box impact test in kN/m.

\begin{tabular}{|c|c|c|c|c|c|c|c|c|}
\hline & $1 X$ & $2 X$ & & $1 Y$ & $2 Y$ & & $1 Z$ & $2 Z$ \\
\hline $1 X$ & 60 & -73 & $1 Y$ & 880 & -830 & $1 Z$ & 290 & -300 \\
\hline $2 X$ & -73 & 73 & $2 Y$ & -830 & 230 & $2 Z$ & -300 & 330 \\
\hline
\end{tabular}

In combining our knowledge of the simplest lumped parameter model that will achieve the necessary motion and FRF matrix, and Equation 4.1.2 which shows the stiffness matrix of the system seen in Figure 4.4, we determined that the connecting spring, $\mathrm{k}_{3}$, is the dominant spring in the system.

$$
\left[\begin{array}{cc}
k_{1}+k_{3} & -k_{3} \\
-k_{3} & k_{2}+k_{3}
\end{array}\right]
$$

From Equation 4.1.2, we saw that although $\mathrm{k}_{1}$ and $\mathrm{k}_{2}$ are not equal, they are soft in comparison to $\mathrm{k}_{3}$. Therefore, we focused on matching the stiffness ratio present in $\mathrm{k}_{3}$ with the intention of tuning $\mathrm{k}_{1}$ and $\mathrm{k}_{2}$ for improvements in the fixture design that would result in closer matching natural frequencies and overall stiffness of the fixture to the nextstructure (Box). We calculated the stiffness ratio seen in Table 4.3 of $\mathrm{k}_{3}$ using Equation 4.1.2.

Table 4.3 Stiffness ratio of connecting spring, $\mathrm{k} 3$, used to begin initial dynamic test fixture design

\begin{tabular}{cc|c}
\hline Direction & Value $(\mathrm{kN} / \mathrm{m})$ & Ratio \\
\hline $\mathbf{k}_{3 \mathrm{X}}$ & 73 & $\mathbf{1}$ \\
\hline $\mathbf{k}_{3 Z}$ & 310 & $\mathbf{3}$ \\
\hline $\mathbf{k}_{3 \mathrm{Y}}$ & 830 & $\mathbf{1 2}$ \\
\hline
\end{tabular}


The stiffness ratio described was used to determine the necessary lengths for a rubber block representing $\mathrm{k}_{3}$. We chose to use a rubber block because it would be easy cut to the length specifications of its directional stiffness's based on the expected load case from understanding the motion of the box.

\subsubsection{Finite Element Model}

The load case for the connecting spring, $\mathrm{k}_{3}$, was determined from our understanding of the dynamics of the box. The overall motion of the fixture needed to be similar, and the fixture design resulted from the knowledge gained from the modal tests that were performed on the box. Figure 4.5 shows the load case defined for the block, with compression in the $\mathrm{X}$ direction, and shear in the $\mathrm{Y}$ and $\mathrm{Z}$ directions.

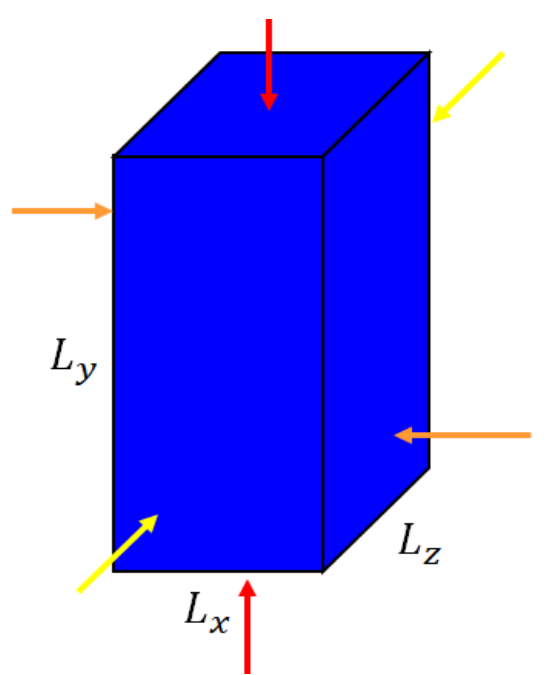

Figure 4.5 Load case defined for connecting spring, $\mathrm{k}_{3}$, rubber block: $\mathrm{Y}$ (Compression/Tension), $\mathrm{X}$ (Shear), Z (Shear).

Once the load case was defined, I used mechanics of materials equations to determine the proper length of each side of the block to produce a similar stiffness values as defined by the box. The calculations used to solve for $L_{x}, L_{y}$, and $L_{z}$ are seen in Equations 4.1.3, 4.1.4, and 4.1.5, where $E$ is the elastic modulus of rubber $\left(40000 \mathrm{kN} / \mathrm{m}^{2}\right)$. 


$$
\begin{gathered}
k_{3 x}=\frac{12 E\left(\frac{1}{12} L_{z} L_{x}^{3}\right)}{L_{y}^{3}}=73 \frac{\mathrm{kN}}{\mathrm{m}} \\
k_{3 y}=\frac{L_{x} L_{z} E}{L_{y}}=830 \frac{\mathrm{kN}}{\mathrm{m}} \\
k_{3 z}=\frac{12 E\left(\frac{1}{12} L_{x} L_{z}^{3}\right)}{L_{y}^{3}}=310 \frac{\mathrm{kN}}{\mathrm{m}}
\end{gathered}
$$

The results of the above calculations lead to the results seen in Table 4.4. This shows the desired lengths of each side of the connecting "spring" to produce similar stiffness to the box.

Table 4.4 Required stiffness and length values for the three sides of the connecting "spring" for the dynamic test fixture design to produce similar stiffness to the box.

\begin{tabular}{ccc}
\hline Direction & $\begin{array}{c}\text { Required } \\
\text { Stiffness }(\mathbf{k N} / \mathbf{m})\end{array}$ & $\begin{array}{c}\text { Length } \\
\text { Value }(\mathbf{m m})\end{array}$ \\
\hline $\mathrm{X}$ & 73 & 34 \\
\hline $\mathrm{Y}$ & 830 & 115 \\
$\mathrm{Z}$ & 310 & 70 \\
\hline
\end{tabular}

After determining these lengths for the sides of the dominant spring, we were able to perform a finite element analysis to verify that we have a similar directional stiffness ratio to the box. Each loading condition resulted in a different analysis, which may have skewed the stiffness results. The FEM of the loading in the three directions is seen in Figure 4.6. 

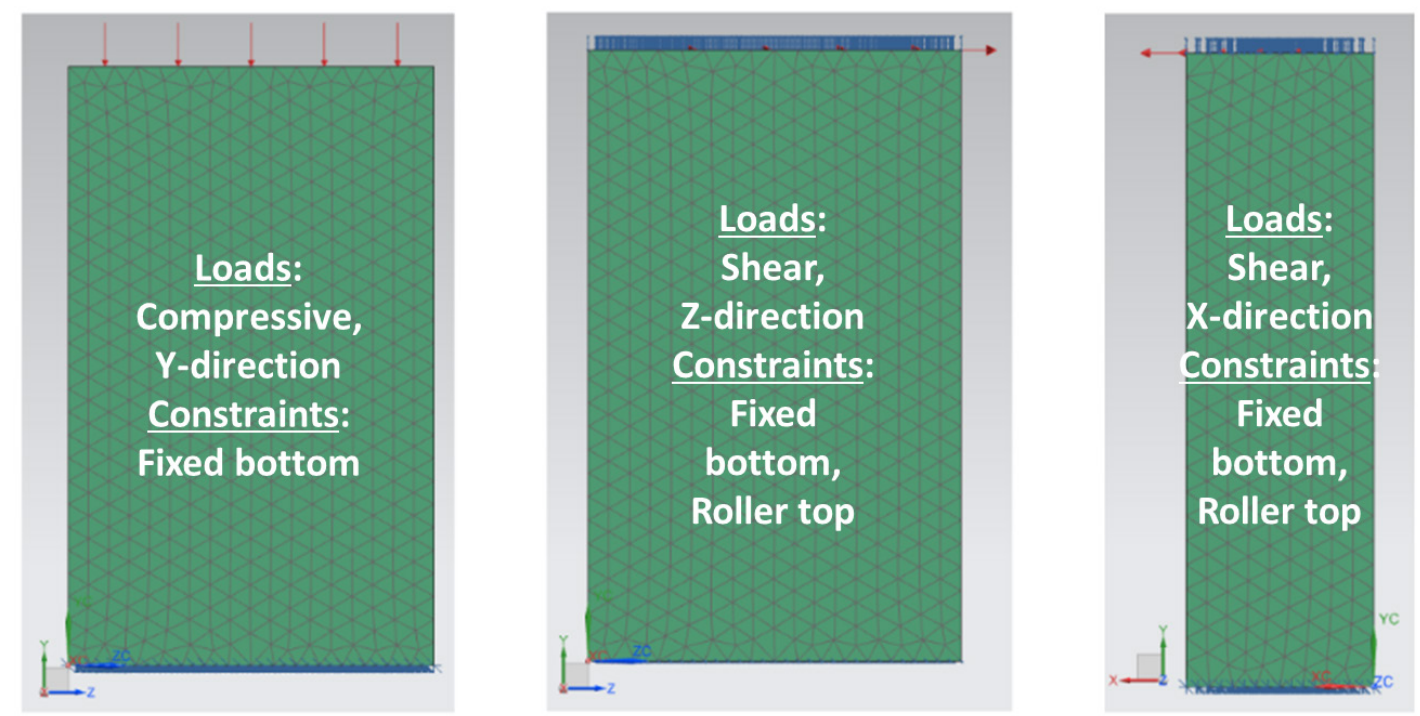

Figure 4.6 Finite Element Model of the connecting spring with specified loading and constraint conditions.

The results of the FEA are seen in Figure 4.7, where $\delta$ is the displacement in a single direction and $k$ is the stiffness calculated from force applied over displacement.
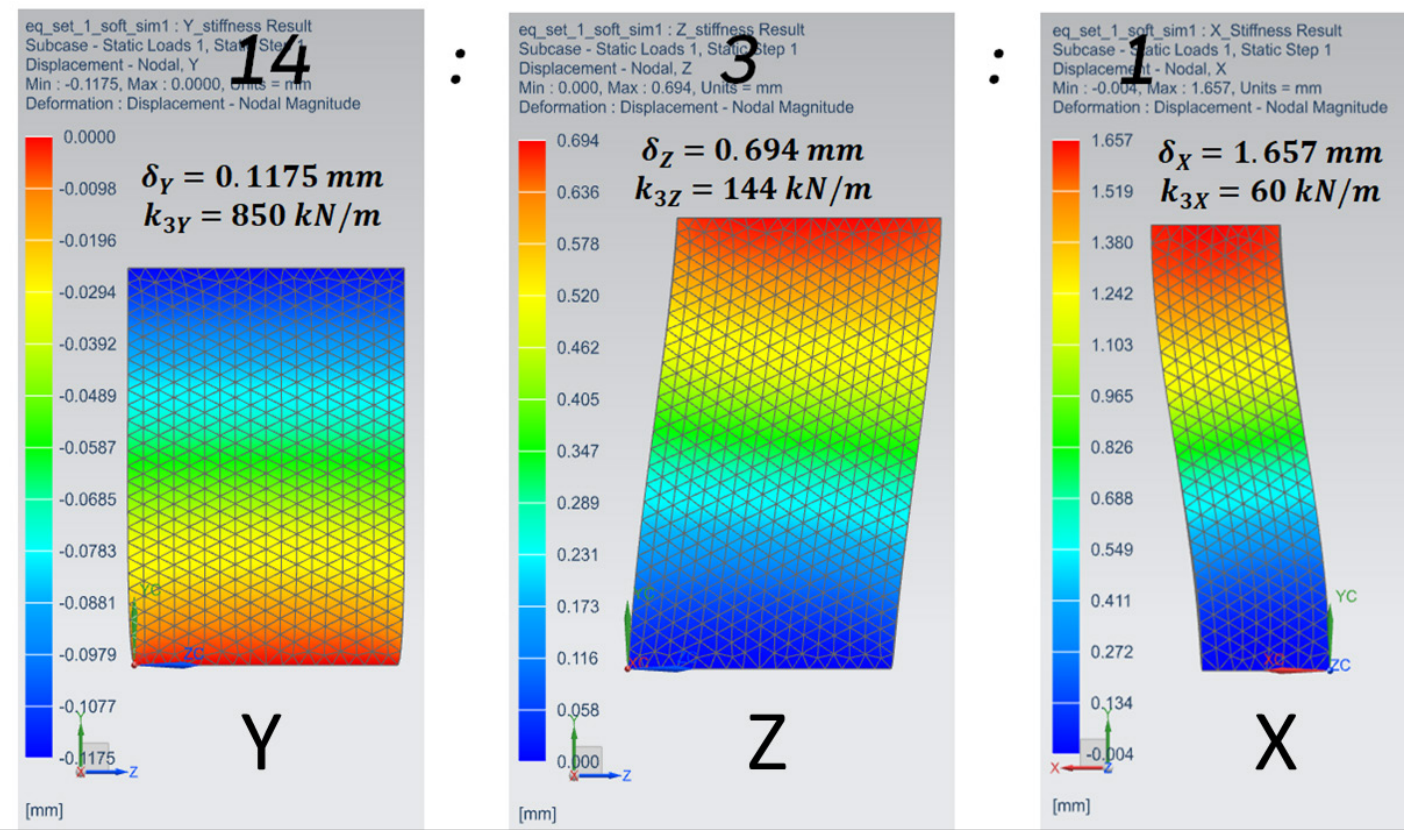

Figure 4.7 Displacement results and stiffness Ratio from the Finite Element Analysis performed on the proposed model of $k_{3}$. 
The stiffness ratio from the FEM is similar to the one defined from the box response. The box stiffness ratio, Y: Z: X, was 12: 3:1, while the rubber block was 14: 3: 1. This result was deemed acceptable enough to continue the design process, with the intention of using $\mathrm{k}_{1}$ and $\mathrm{k}_{2}$ to adjust for the stiffness discrepancy in $\mathrm{k}_{3}$ between the fixture and the nextstructure. Some requirements of the fixture design included:

- Mimic the response at the attachment points of the free-free box.

- Hold the rubber block $\mathrm{k}_{3}$.

- Mount to the large shaker table.

- Component must be able to attach to it.

The original fixture design can be seen in Figure 4.8. With the understanding that tuning of the rubber pillars, springs $k_{1}$ and $k_{2}$, would be necessary we decided to move forward with this design.
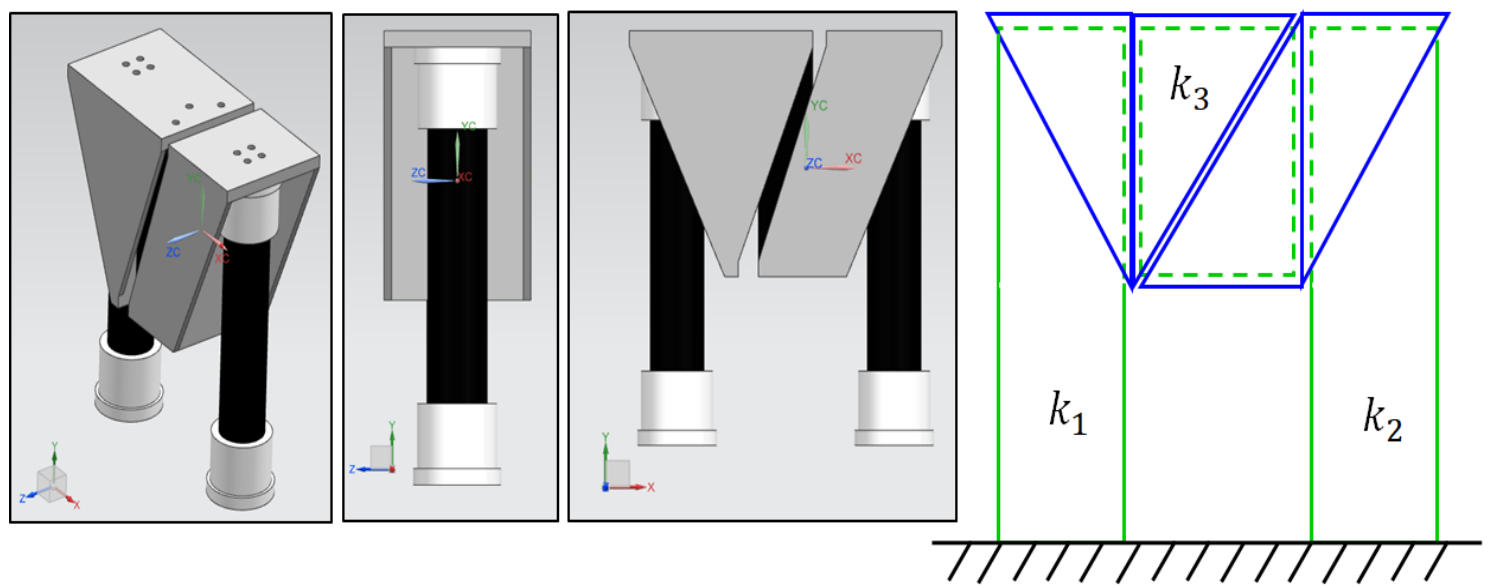

Figure 4.8 Original dynamic test fixture design as a result of the stiffness values retrieved from the attachment locations on the free-free box.

I created a FEM of the design, seen in Figure 4.9, to predict the fixture accelerance response prior to physical testing. The base of each rubber pillar is fixed to ground, while all other connections are glued. We also made a FEM of the box, seen in Figure 4.9, to allow for comparison of the attachment FRFs between the two structures. We made the box model with shell elements with defined thickness, while the fixture had 3D tetra elements based on the geometry of the structure. 

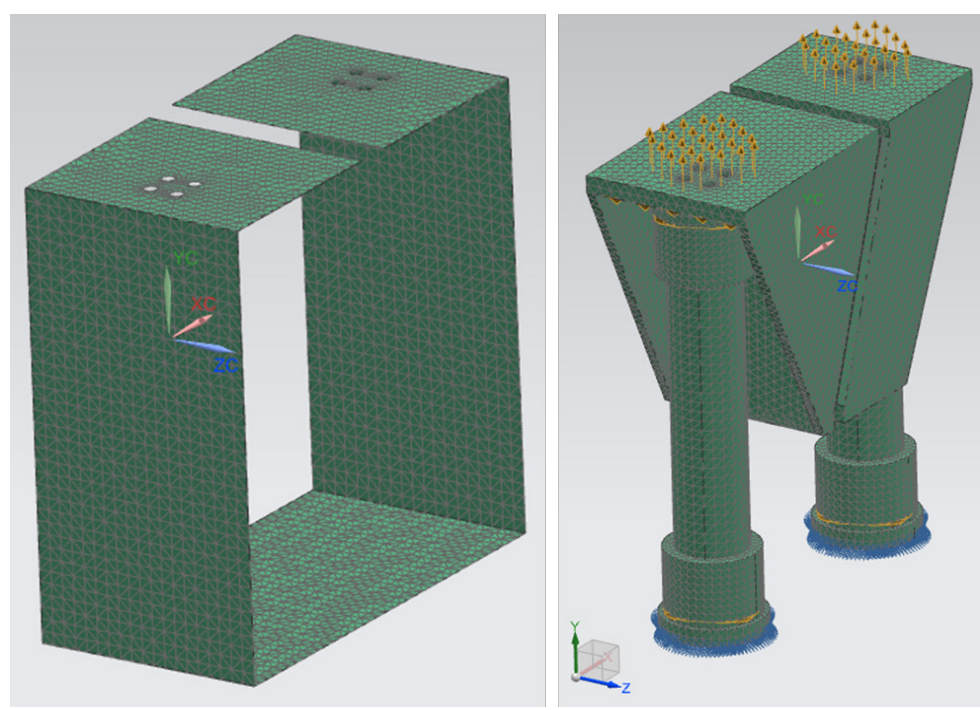

Figure 4.9 Finite Element Models of Box in free-free condition and dynamic fixture design with fixed base constraints.

Figure 4.10 shows one of the comparable mode shapes. We saw that the motion and amplitude was similar, although the natural frequencies/locations of the modes were not the same. This was expected, since we performed no analysis on $k_{1}$ or $k_{2}$ to adjust for the stiffness discrepancies in $k_{3}$ between the box and the fixture we designed.

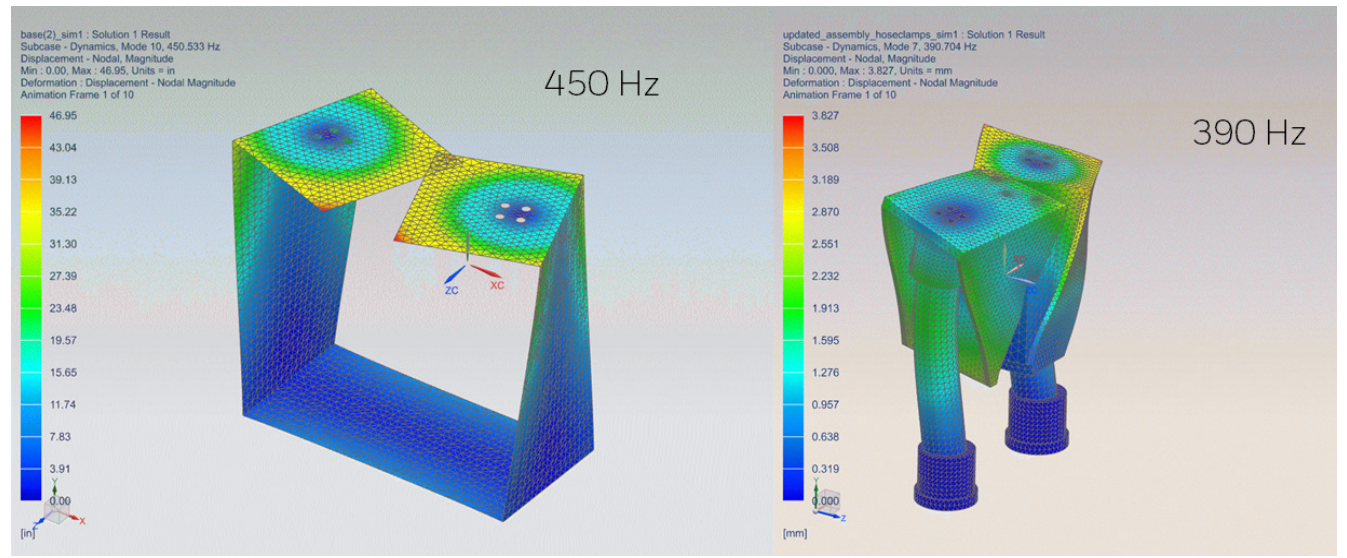

Figure 4.10 Box vs. Fixture (Mode 10 vs. Mode 7) where we see the difference in frequency but overall similarity in motion.

In addition to seeing if the box and dynamic fixture produce similar relative motion between the attachment locations, we also wanted to compare their general stiffness trends. Figure 4.11, shows the FRFs from the two attachment locations of the box (blue) and the 
fixture (orange) in the Y-direction, with 1\% damping. We looked at the relationship between the two in all three directions, but we were really only concerned with the Ydirection, as it is the excitation direction of structures attached to the shaker. The FRFs from the $\mathrm{X}$ - and Z-directions can be found in 9A.

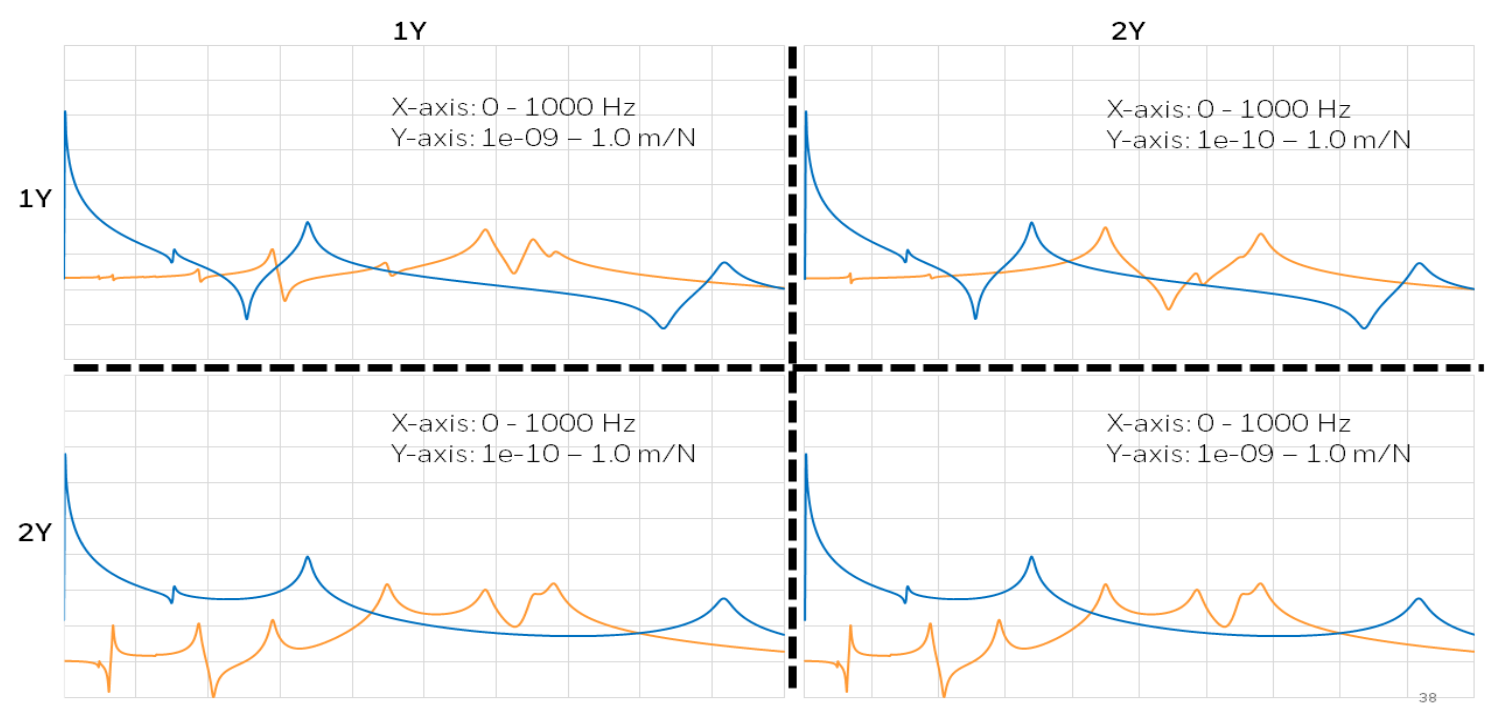

Figure 4.11 Y-directional accelarance plots from the fixture (Orange) and box (Blue).

Overall, the general stiffness trends between the box and the fixture are similar. However, the fixture has more resonances than the box, and the locations of these resonances are not near those of the box. This showed us that the fixture may provide a similar stiffness and relative motion, but with the modes and attachment FRFs not matching, the fixture would not produce the target response desired on the COI. Due to the difference in BCs between the box and fixture, we see the rigid body modes of the box at $0 \mathrm{~Hz}$.

\subsubsection{Hardware}

Taking a design to physicality can be a challenging task. One of the main goals of this fixture design was to have similar mass to the box. To accomplish this, we decided to 3D print the connecting brackets that hold the rubber block and pillars together and attaches to the component. From the stiffness analysis we knew that we would need as stiff of rubber pillars as possible, so we used the $90 \mathrm{~A}$ ones we had in the lab. The final fixture can be seen 
in Figure 4.12, where the rubber pillars are polyurethane with PVC caps for attachments, and the brackets are made of ABS plastic.

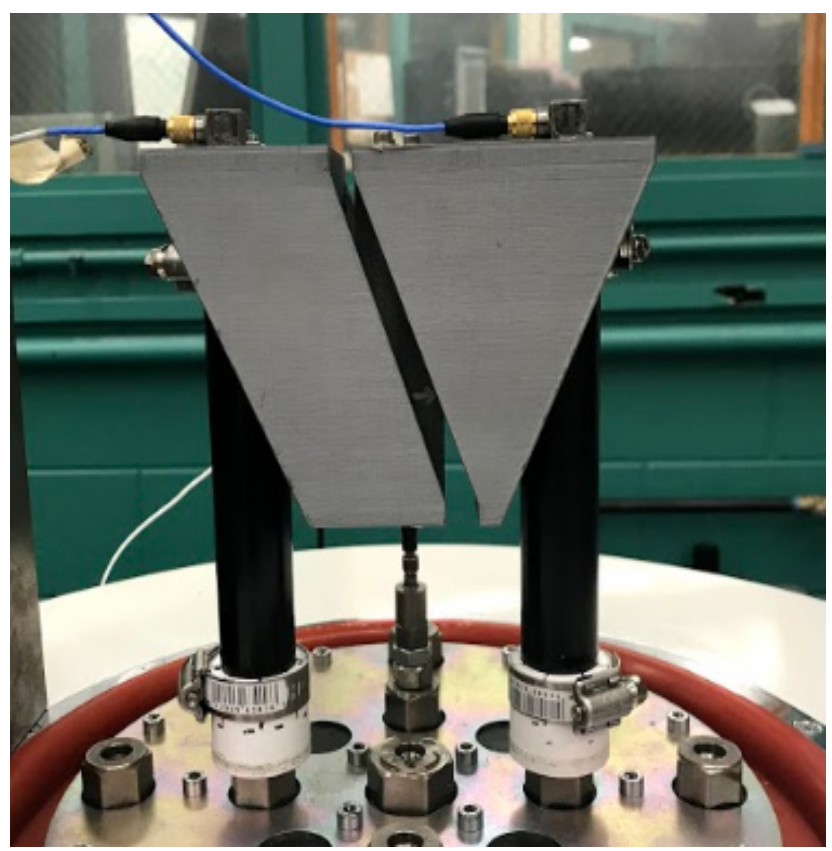

Figure 4.12 Final fixture design, with AM brackets, rubber pillars, and rubber block in the center. 


\section{Physical Testing}

In this chapter we discuss the testing performed on the BARC and dynamic test fixture. We collected attachment FRFs from the free-free box and from a fixed box. Then performed two validation methods to determine the potential success of the fixture in reproducing the next-structure response. One method is based on truth data collected from the free-free box and the other is based on rocket field data from the fixed box.

\subsubsection{Experimental Analysis}

We decided to move on to physically testing the fixture in relation to the box, with plans of adjusting frequency locations in the future.

\subsubsection{Test 1: Attachment FRFs}

We gathered the attachment FRFs from both the box in a free-free condition and the dynamic fixture in a fixed condition. The free-free box condition was mentioned in Chapter 4, where we discussed the initial fixture design. The free-free box FRFs were used to design the dynamic test fixture. Both structures were tested by providing an impact source in the $\mathrm{X}, \mathrm{Y}$, and Z-directions near the attachments and obtaining the response from two tri-axial accelerometers at these attachments. Schematics of the tests conducted can be seen in Figure 5.1.


Figure 5.1 Attachment FRF collection test for next-structure and dynamic test fixture. 
The goal of this test was to identify the success of the fixture design in replicating the response of the box at the attachments.

\subsubsection{Attachment Time History}

To validate the success of the fixture design and input profile in replicating the next-level assembly response several tests had to be performed. The first step was to define the original input signal. The original input signal is defined from obtaining the attachment time histories from with some base input to the next-structure. This would allow us to use this response as the shaker control for the fixture test.

Since the fixture was designed based on the free-free next-structure, we first collected time histories of the box from a random shaker input to the base of the free-free box. This test setup is realized in Figure 5.2.

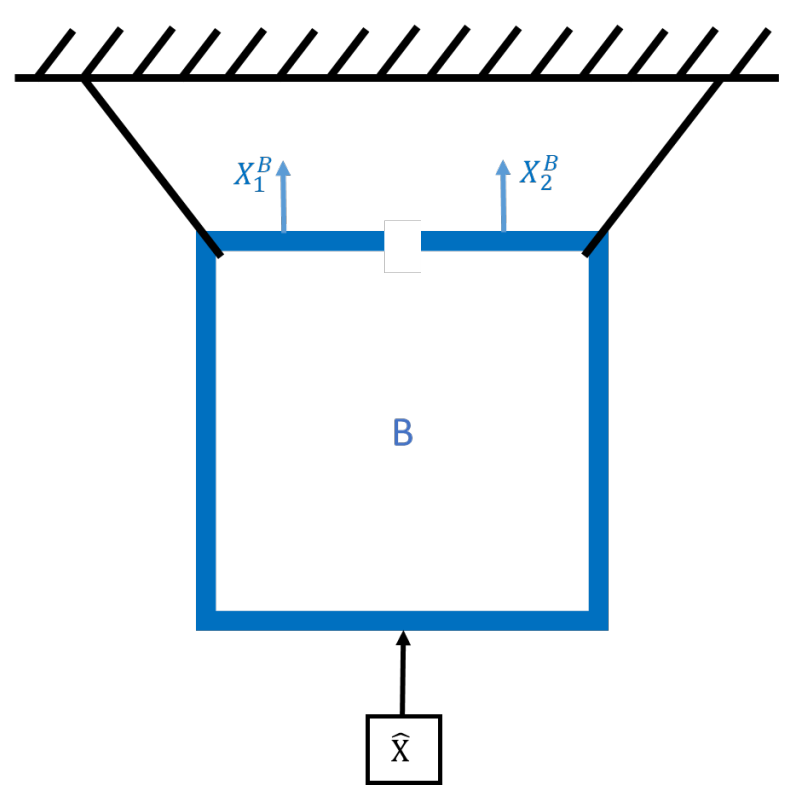

Figure 5.2 Attachment time response collection test for free-free box with random base input.

The second method was gathering the attachment time histories from the fixed box. Honeywell provided us with data that was collected from a flight on which the BARC was attached and instrumented. In using this real flight data we were able to capture the time response at the attachment locations of both the next-structure and the fixture as seen in 
Figure 5.3 and Figure 5.4, where the shaker is being controlled by the signal $\hat{X}$ at the base of the structures.

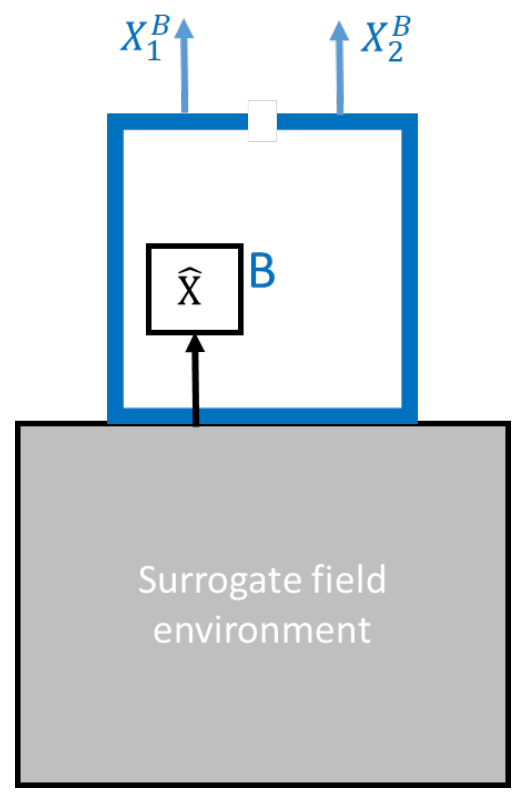

Figure 5.3 Next-structure (box) test set-up used to gather attachment point time histories $\left(X_{1}^{B}\right.$ and $X_{2}^{B}$ ) from both a random and rocket vibration profile with base control.

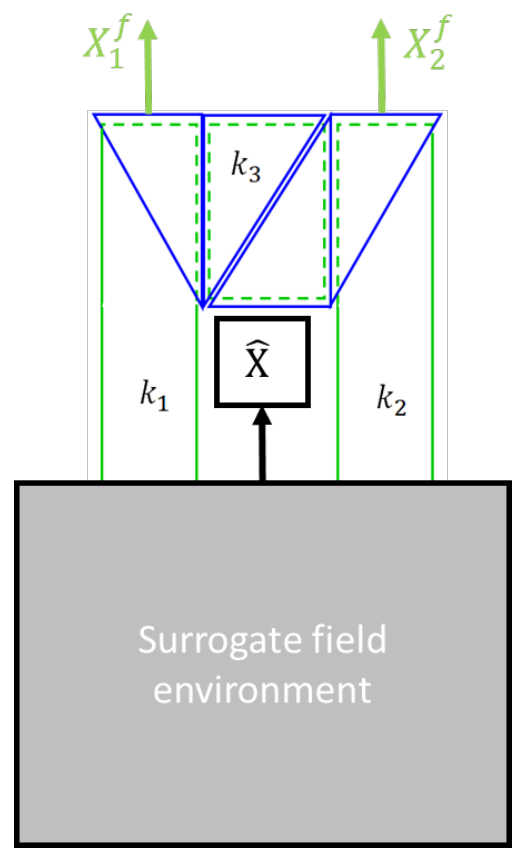

Figure 5.4 Fixture test set-up used to gather attachment point time histories $\left(X_{1}^{f}\right.$ and $\left.X_{2}^{f}\right)$ from both a random and rocket vibration profile with base control. 


\subsubsection{Shaker Voltage Signal}

After collecting the time responses from the attachment locations, we used waveform replication to reproduce this response with the shaker controller. While controlling the shaker off of the attachment locations, we used the memorized drive feature to record the actual voltage signal going to the shaker. Figure 5.5 and Figure 5.6 show test set-up schematics for the next-structure and box.

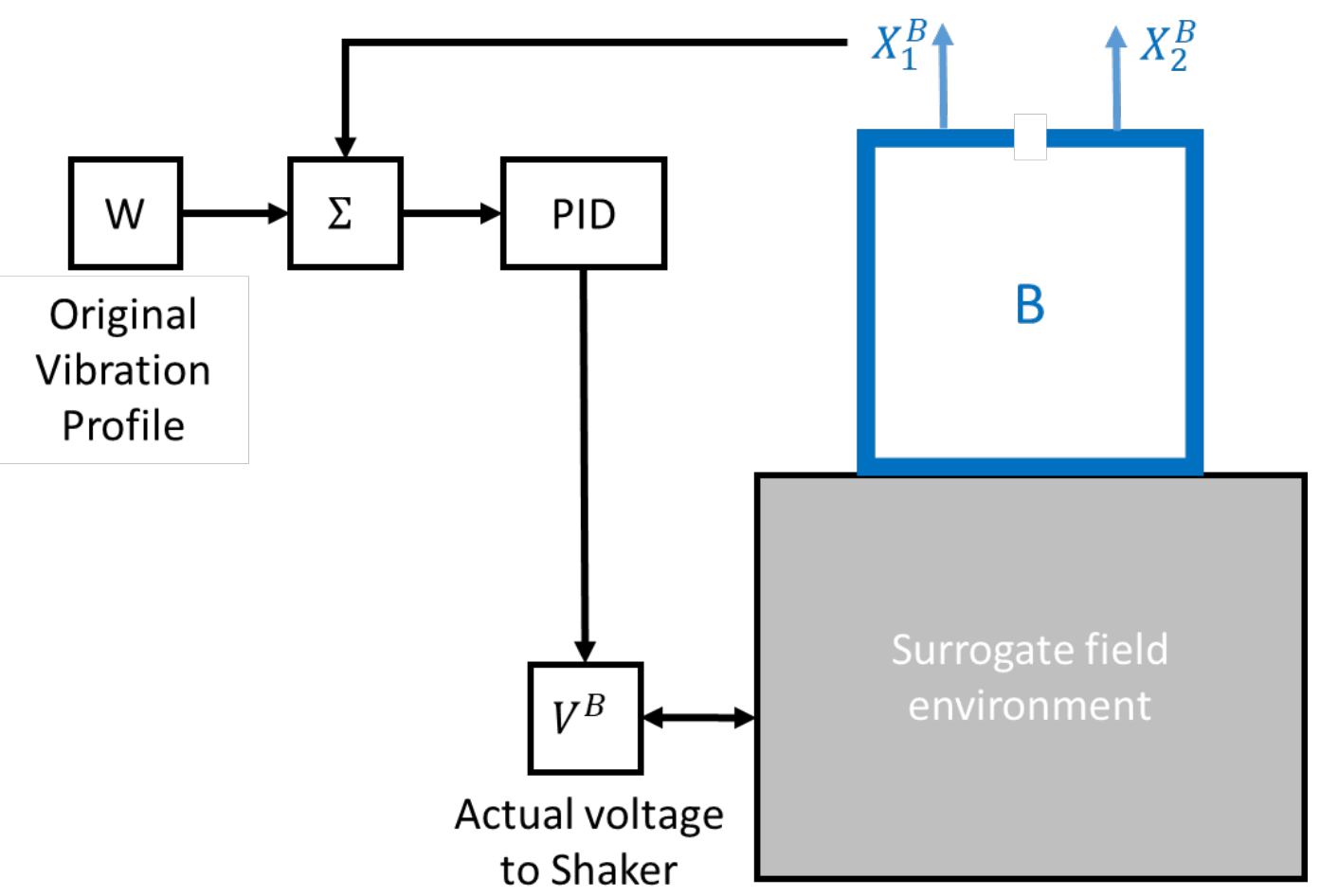

Figure 5.5 Next-structure test set-up used to obtain the voltage signal going to the shaker from controlling at the attachment locations with time response signals from the previous test.

Figure 5.5 shows the test performed for the fixed box condition to obtain the voltage signal. For the free-free box condition we controlled the attachment response for the fixture based on the response obtained from the free-free box test. This allowed us to get the voltage signal necessary to compensate for the differences between the free-free box and the fixture. 


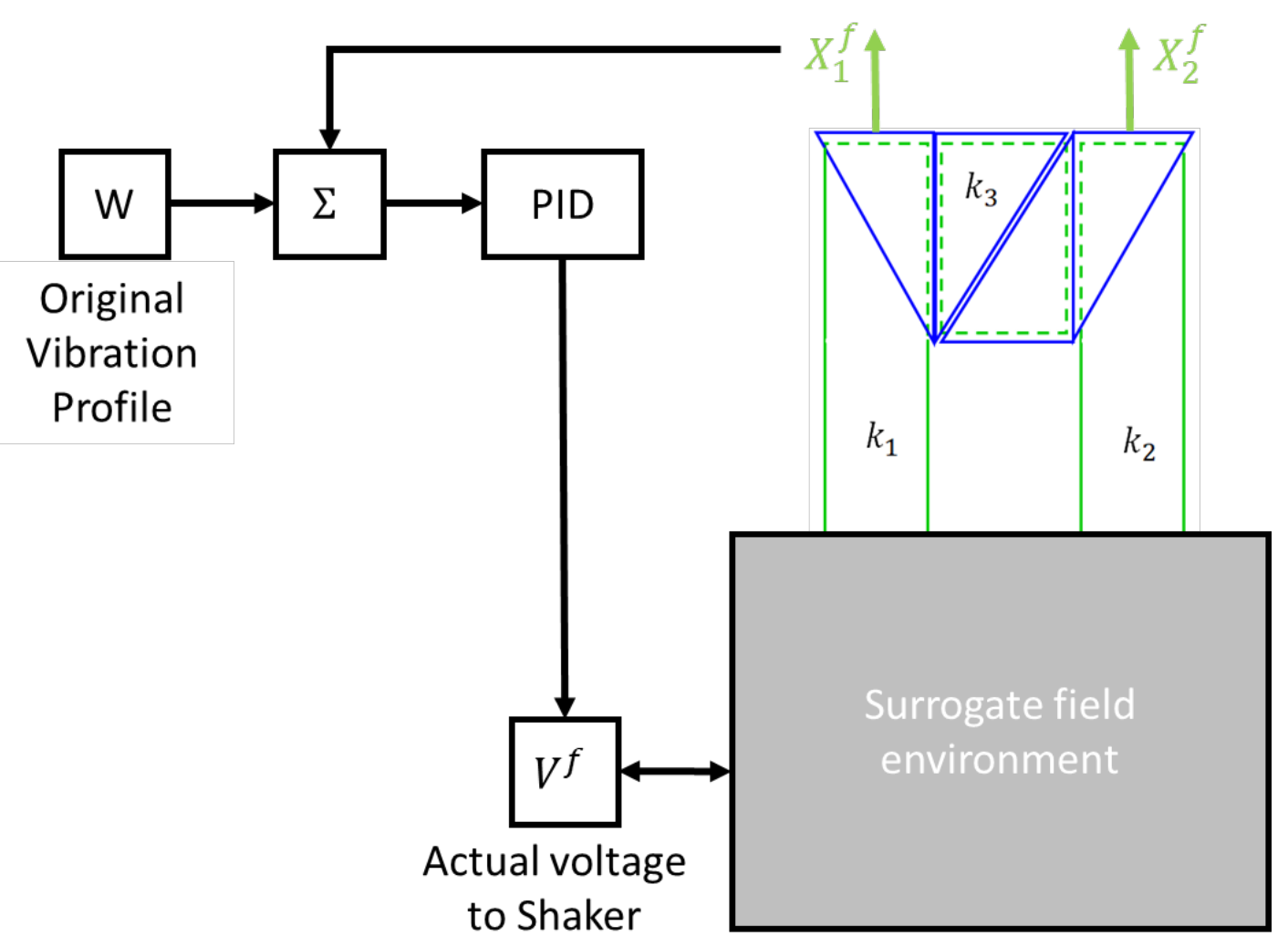

Figure 5.6 Fixture test set-up used to obtain the voltage signal going to the shaker from controlling at the attachment locations with time response signals from the previous test.

The goal of these tests was to get the actual voltage signal going to the shaker to allow us to run open loop tests with the component attached to get its response.

\subsubsection{Component Response}

The next step in the validation procedure was to get the response across the component. This was done for both the free-free box condition and the fixed box. For the free-free box we used the same initial shaker input to the base of the box with the component attached to obtain response, as seen in Figure 5.7. 


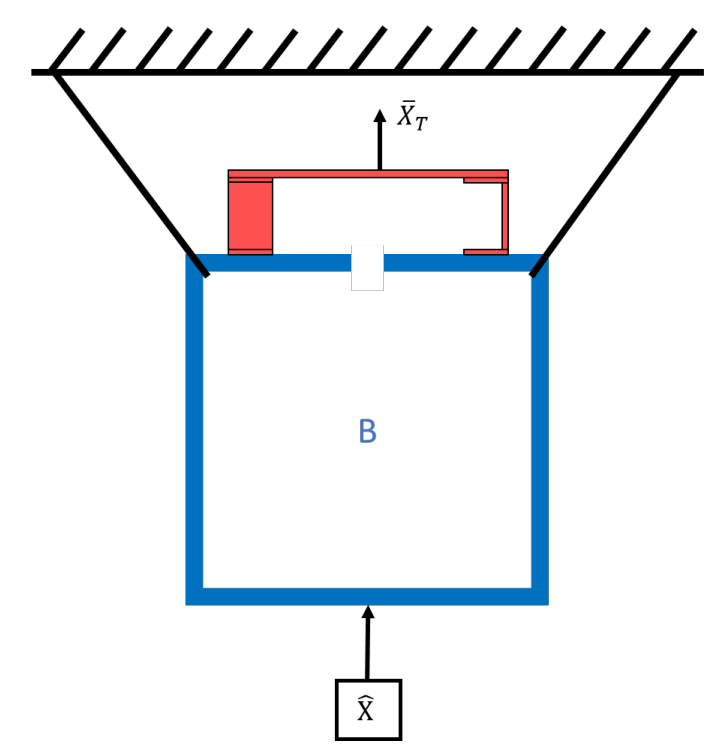

Figure 5.7 Obtaining the component response from free-free box with base input.

For both the free-free and fixed box conditions, we used the memorized drive voltage signals to run the shaker open loop and gather the component time response. Figure 5.8 shows the test setup used to gather the target response for the fixed box and Figure 5.9 shows the test setup used to gather the response of the component on the fixture as a result of either the free-free defined voltage input or fixed voltage input.

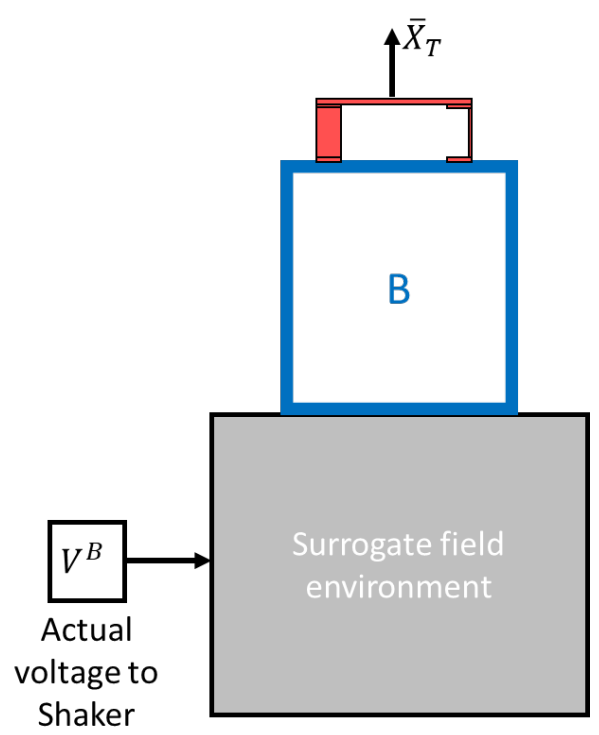

Figure 5.8 Next-level assembly test set-up to gather component response based off voltage input gathered from controlling an attachment location based on attachment time histories. 


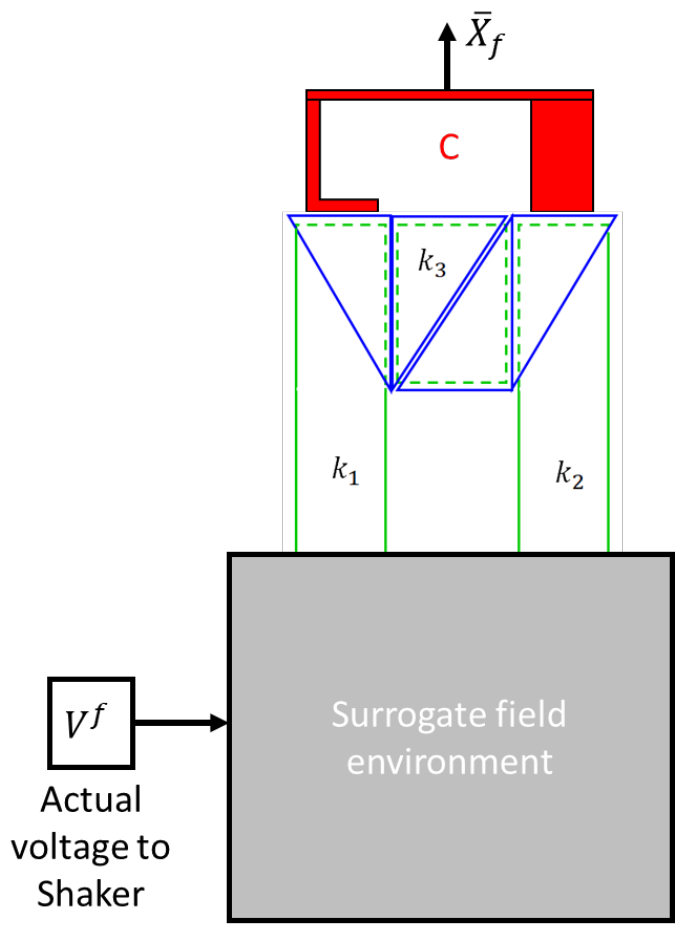

Figure 5.9 Fixture assembly test set-up to gather component response based off voltage input gathered from controlling an attachment location based on attachment time histories.

In all tests, the component was outfitted with 6 small single axis accelerometers and 3 strain gauges as seen in Figure 5.10.



Figure 5.10 Component instrumentation for both target data and fixture response collection. 


\section{Parameter Optimization Method}

This chapter discusses a method for dynamic test fixture design using attachment FRFs to develop a lumped parameter model. The parameters are then optimized and the model is brought into physicality through the use of a pin-pin beam and tuned absorbers.

\subsection{Optimized Fixture Parameters}

As mentioned previously, all structures can be defined by a LPM. We can create a LPM based on our physical knowledge of the structure or by its FRFs. The attachment FRFs collected from the next-level assembly can provide insight for the creation of a model. For this model we chose a single attachment next-structure and component. All validation is performed using the BARC hardware with the box without the cut in the center. For this method, three sets of information are needed from a surrogate field test of the next-structure and component:

- Drive-Point FRFs at attachment DOFs.

- Cross-Point FRFs for multiple Attachment FRFs.

- Mode shape coefficients from FRFs.

The first step in this method is to gather the attachment FRFs. The next-structure was mounted in a fixed condition. A single tri-axial accelerometer was mounted to the center of the next-structure, and an impacts were made in the $\mathrm{X}, \mathrm{Y}$, and $\mathrm{Z}$ directions near the accelerometer to collect drive point FRFs. The following analysis is only performed using the $\mathrm{Y}$-direction, but the $\mathrm{X}$ and $\mathrm{Z}$ directions were collected for future MIMO testing. The test set-up can be seen in Figure 6.1. 


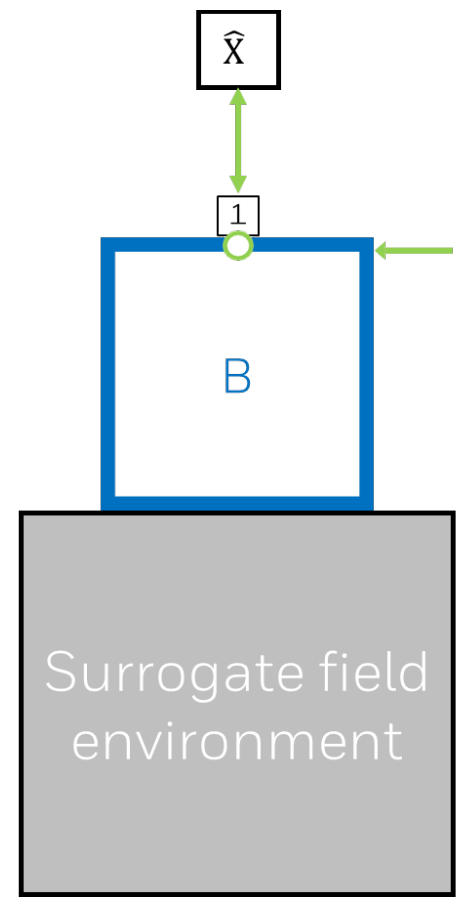

Figure 6.1 Test set-up for gathering attachment FRFs for a single attachment next-structure in its surrogate field environment.

The accelerance and compliance drive point FRFs from the $\mathrm{Y}$ direction impact and response can be seen in Figure 6.2. From this FRF we can identify the number of modes and therefore the number of DOFs or masses needed to accurately represent the structure.

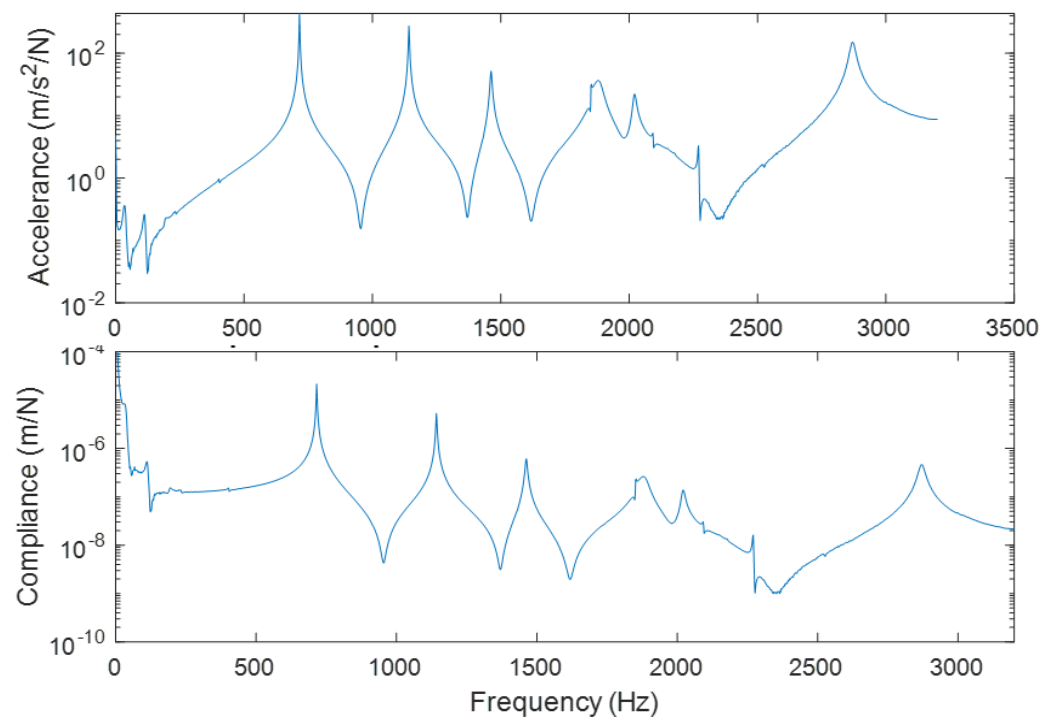

Figure 6.2 BARC Y-direction single attachment FRFs for LPM development. 
Using a curve fitting code we obtain the optimization targets (Table 6.1) from a synthesized FRF (Figure 6.3) representing the next structure.

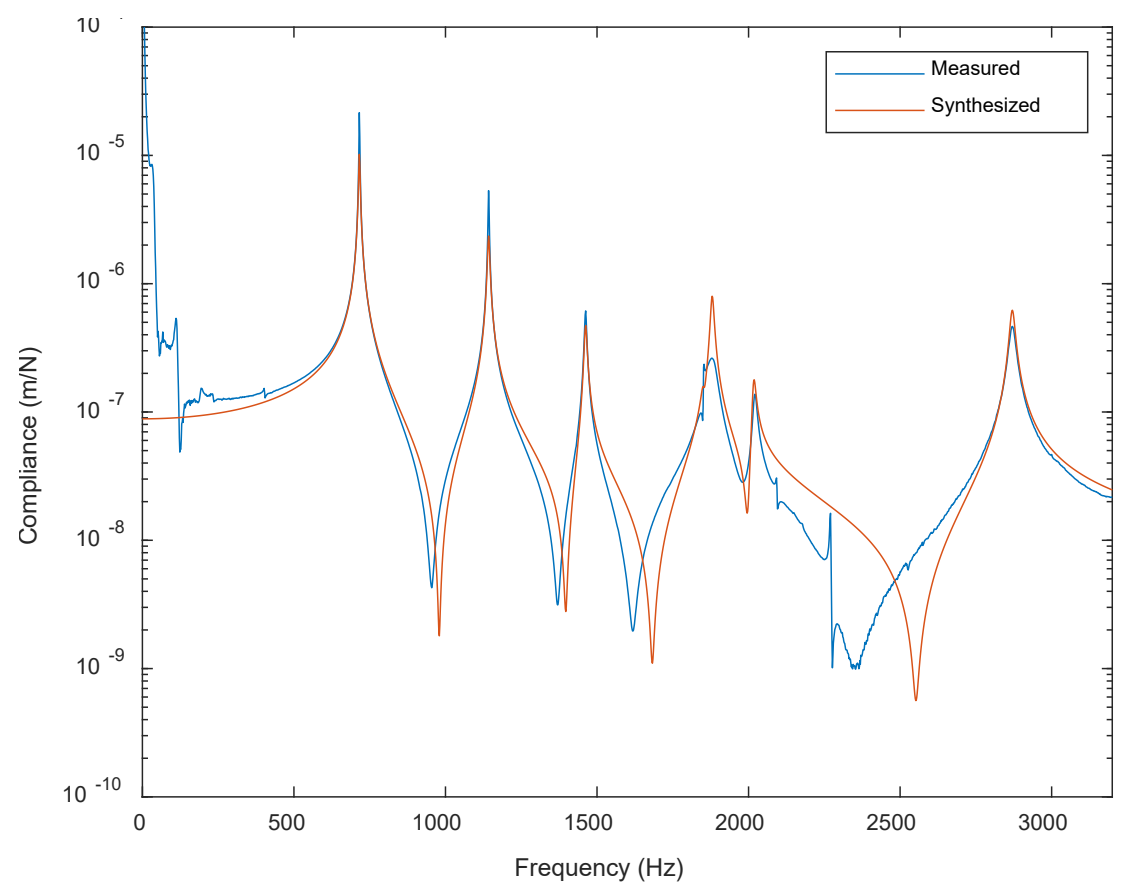

Figure 6.3 Measured and synthesized Y-direction FRFs from the single attachment next-structure.

Table 6.1 Optimization targets from single attachment target FRF.

\begin{tabular}{cccc}
\hline Mode \# & $\begin{array}{c}\text { Mode Shape } \\
\text { Coefficient }\end{array}$ & $\begin{array}{c}\text { Eigen Value } \\
(\mathbf{r a d} / \mathbf{s e c})^{\mathbf{2}}\end{array}$ & $\begin{array}{c}\text { Natural Frequency } \\
\mathbf{( H z )}\end{array}$ \\
\hline $\mathbf{1}$ & 1.1173 & $2.024 \mathrm{E}+07$ & 716 \\
\hline $\mathbf{2}$ & 0.8527 & $5.151 \mathrm{E}+07$ & 1142 \\
\hline $\mathbf{3}$ & 0.4882 & $8.440 \mathrm{E}+07$ & 1462 \\
\hline $\mathbf{4}$ & 0.183 & $1.353 \mathrm{E}+08$ & 1851 \\
\hline $\mathbf{5}$ & 0.8173 & $1.395 \mathrm{E}+08$ & 1880 \\
\hline $\mathbf{6}$ & 0.4008 & $1.606 \mathrm{E}+08$ & 2017 \\
\hline $\mathbf{7}$ & 1.1003 & $3.252 \mathrm{E}+08$ & 2870 \\
\hline
\end{tabular}

This FRF and target values are used to calculate error between the optimized FRFs from the lumped parameter fixture model and iterate the optimizer to meet them. The model is used to obtain the values for the mass, stiffness, and damping parameters for a successful single attachment, single direction fixture design (Figure 6.4). The model should be as simple as possible while satisfying the following criteria: 
- The model possesses the same relative stiffness between the component and model as that of the component and next-structure.

- The model has the same number of attachment DOFs as the next-level assembly.

- The model has the same number of modes in the frequency band as the next-level assembly.

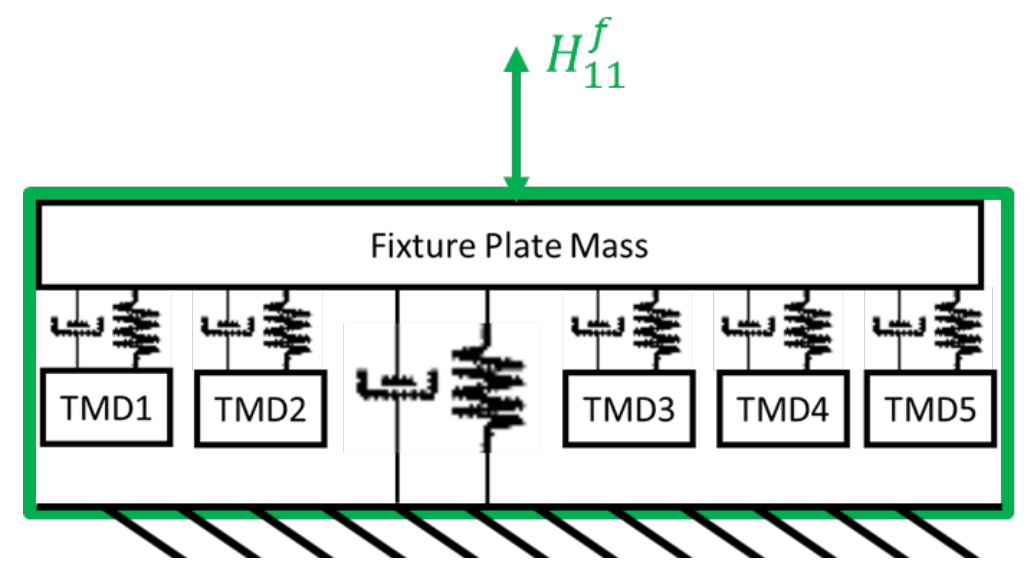

Figure 6.4 Lumped parameter model used to determine the parameters needed to match the target attachment FRF.

We retrieve the FRF at the "fixture plate mass" where the COI would be attached. An optimizer with a least squares objective function was developed to iterate until the FRF from the fixture LPM seen in Figure 6.4, until the parameters of the LPM produce a similar FRF to the target. The optimizer exponentially scales the eigenvalues to have weighed values within one order of magnitude, and the mode shape coefficients were scaled equally to have values within the same order of magnitude as weighted eigenvalues. Initial values to enter into the optimization are determined from the target FRF and knowing the mass of the next-structure. We divide the mass of the next-structure between the masses of the LPM. The main spring stiffness is calculated by inverting the compliance FRF at $0 \mathrm{~Hz}$, and the damping was ignored. The iterated FRFs and $\mathrm{m}, \mathrm{c}$, and $\mathrm{k}$ values can be seen in Figure 6.5 and Table 6.2, respectively. 
Table 6.2 Optimizer iterated values for the tuned absorber parameters from fixture LPM of single attachment next-structure.

\begin{tabular}{|c|c|c|c|c|c|}
\hline & & Seed Values & Iteration 1 & Iteration 2 & Iteration 3 \\
\hline \multirow{7}{*}{  } & Base & 1.0000 & 0.5766 & 0.3684 & 0.2358 \\
\hline & $T M D 1$ & 0.1000 & 0.1740 & 0.1716 & 0.0839 \\
\hline & $T M D 2$ & 0.1000 & 0.0655 & 0.0667 & 0.0301 \\
\hline & $T M D 3$ & 0.1000 & 0.1231 & 0.1434 & 0.0480 \\
\hline & $T M D 4$ & 0.1000 & 0.0003 & 0.0003 & 0.0003 \\
\hline & $T M D 5$ & 0.1000 & 0.0932 & 0.0049 & 0.0029 \\
\hline & $T M D 6$ & 0.1000 & 0.1872 & 0.0841 & 0.0411 \\
\hline \multirow{7}{*}{ 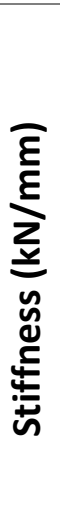 } & Base & 20.2354 & 29.6316 & 22.0788 & 11.3244 \\
\hline & $T M D 1$ & 5.1512 & 7.3523 & 6.7190 & 3.2400 \\
\hline & $T M D 2$ & 8.4396 & 5.1687 & 5.2690 & 2.3003 \\
\hline & $T M D 3$ & 13.5299 & 15.6128 & 14.3786 & 5.4435 \\
\hline & $T M D 4$ & 13.9534 & 0.0412 & 0.0367 & 0.0357 \\
\hline & $T M D 5$ & 16.0568 & 14.6770 & 0.7610 & 0.4546 \\
\hline & $T M D 6$ & 32.5201 & 36.8767 & 18.8014 & 10.5094 \\
\hline
\end{tabular}




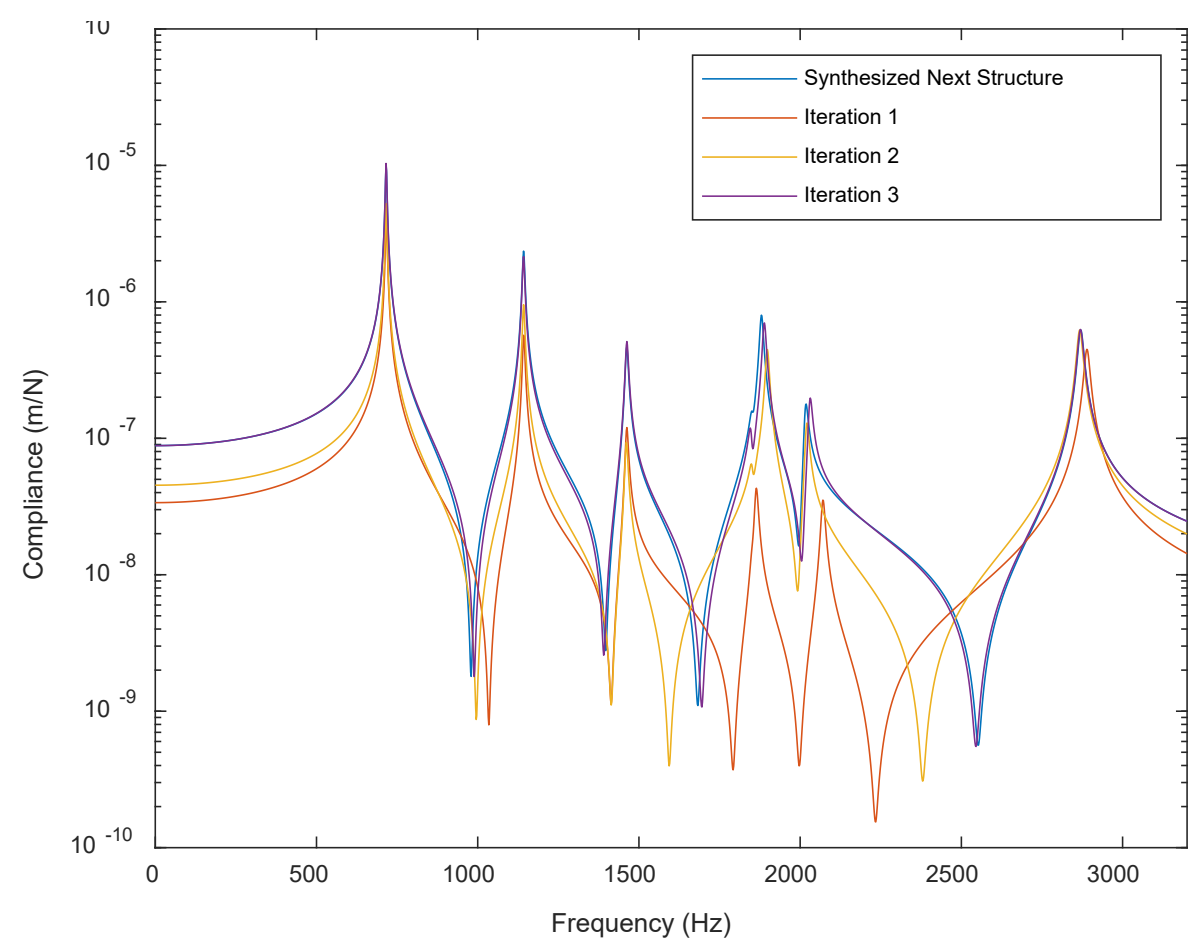

Figure 6.5 Optimizer iterated FRFs from fixture LPM of single attachment next-structure.

\subsection{Designing the Fixture}

The final iteration provides a FRF that is nearly an exact match to the next-structure FRF. The values from this iteration were used to begin the design of a test fixture that will attach a single attachment component to the shaker. We decided a pin-pin, fixed-fixed, or cantilevered beam with tuned absorbers would be the simplest to design and manufacture.

The mass and stiffness of the main beam were defined by the optimization. Three tests, one of each condition were analytically tested. The width and thickness were limited to 1 " $(0.0254 \mathrm{~m})$ and $0.5 "(0.0127 \mathrm{~m})$, respectively. The material parameters were based on steel with an elastic modulus of $2.07 \mathrm{E}+11$ and a density of $7700 \mathrm{~kg} / \mathrm{m}^{3}$. The results are seen in Table 6.3 where we see the length of the beam and additional mass required to produce the required stiffness and mass properties. 
Table 6.3 Results of 3 tests to determine which type of BCs the main beam should have.

\section{Cantilivered}

\begin{tabular}{|lcccc|}
\hline Length & 0.062 & $\mathrm{~m}$ & 2.44 & inch \\
Modal Factor & 4953 & & & \\
Natural Frequency & 17385 & $\mathrm{rad} / \mathrm{sec}$ & 2767 & $\mathrm{~Hz}$ \\
Beam Modal Mass & 0.04 & $\mathrm{~kg}$ & & \\
Additional Mass & 0.198 & $\mathrm{~kg}$ & & \\
\hline
\end{tabular}

Pin-pin
\begin{tabular}{|lcccc|}
\hline Length & 0.156 & $\mathrm{~m}$ & 6.15 & inch \\
Modal Factor & 780 & & & \\
Natural Frequency & 7699 & $\mathrm{rad} / \mathrm{sec}$ & 1225 & $\mathrm{~Hz}$ \\
Beam Modal Mass & 0.19 & $\mathrm{~kg}$ & & \\
Additional Mass & 0.045 & $\mathrm{~kg}$ & & \\
\hline
\end{tabular}

\section{Fixed-Fixed}

\begin{tabular}{|lcccc|}
\hline Length & 0.248 & $\mathrm{~m}$ & 9.76 & inch \\
Modal Factor & 309.5562 & & & \\
Natural Frequency & 6925 & $\mathrm{rad} / \mathrm{sec}$ & 1102 & $\mathrm{~Hz}$ \\
Beam Modal Mass & 0.24 & $\mathrm{~kg}$ & & \\
Additional Mass & 0.000 & $\mathrm{~kg}$ & & \\
\hline
\end{tabular}

Since the fixed-fixed beam does not require additional mass to produce the necessary stiffness requirements, it is the best boundary condition to be used for this fixture design. This condition also allows for enough space for the additional tuned absorbers needed for the modes of the fixture, seen in Figure 6.6.

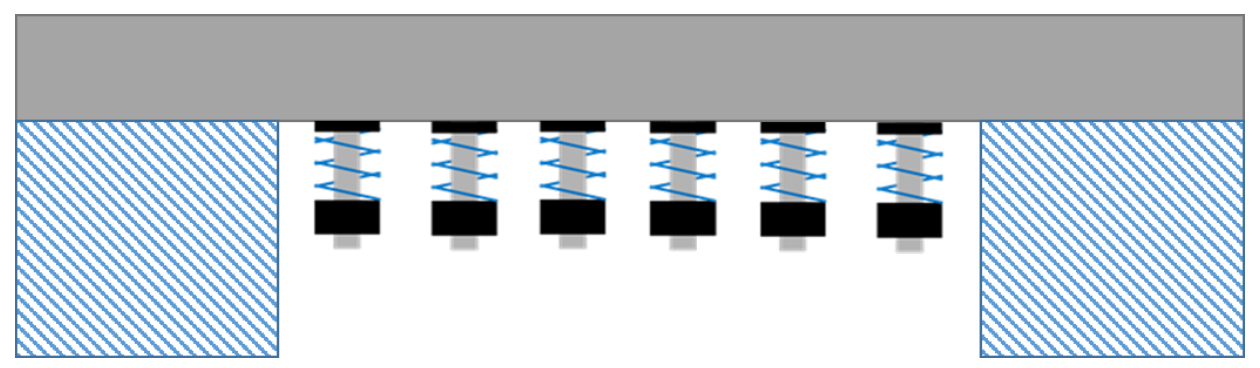

Figure 6.6 Fixed-fixed beam with tuned absorbers to represent the LPM of the fixture.

Using the optimization algorithm we were able to design the LPM with the TMD parameters seen in 
Table 6.4.

Table 6.4 Lumped Parameter Model tuned absorber mass and stiffness properties.

\begin{tabular}{ccc} 
& Mass (grams) & Stiffness $(\mathbf{k N} / \mathbf{m m})$ \\
\hline TMD 1 & 84 & 3.240 \\
\hline TMD 2 & 30 & 2.300 \\
\hline TMD 3 & 48 & 5.444 \\
\hline TMD 4 & 0 & 0.036 \\
\hline TMD 5 & 3 & 0.455 \\
\hline TMD 6 & 41 & 10.509 \\
\hline
\end{tabular}

From these parameters we developed a finite element model of the fixture LPM. The FEM is shown in Figure 6.7, where the ends are fixed and the TMDs are all in the center of the $1 \mathrm{D}$ element beam.



Figure 6.7 Finite Element Model of fixture LPM from optimized parameters. 


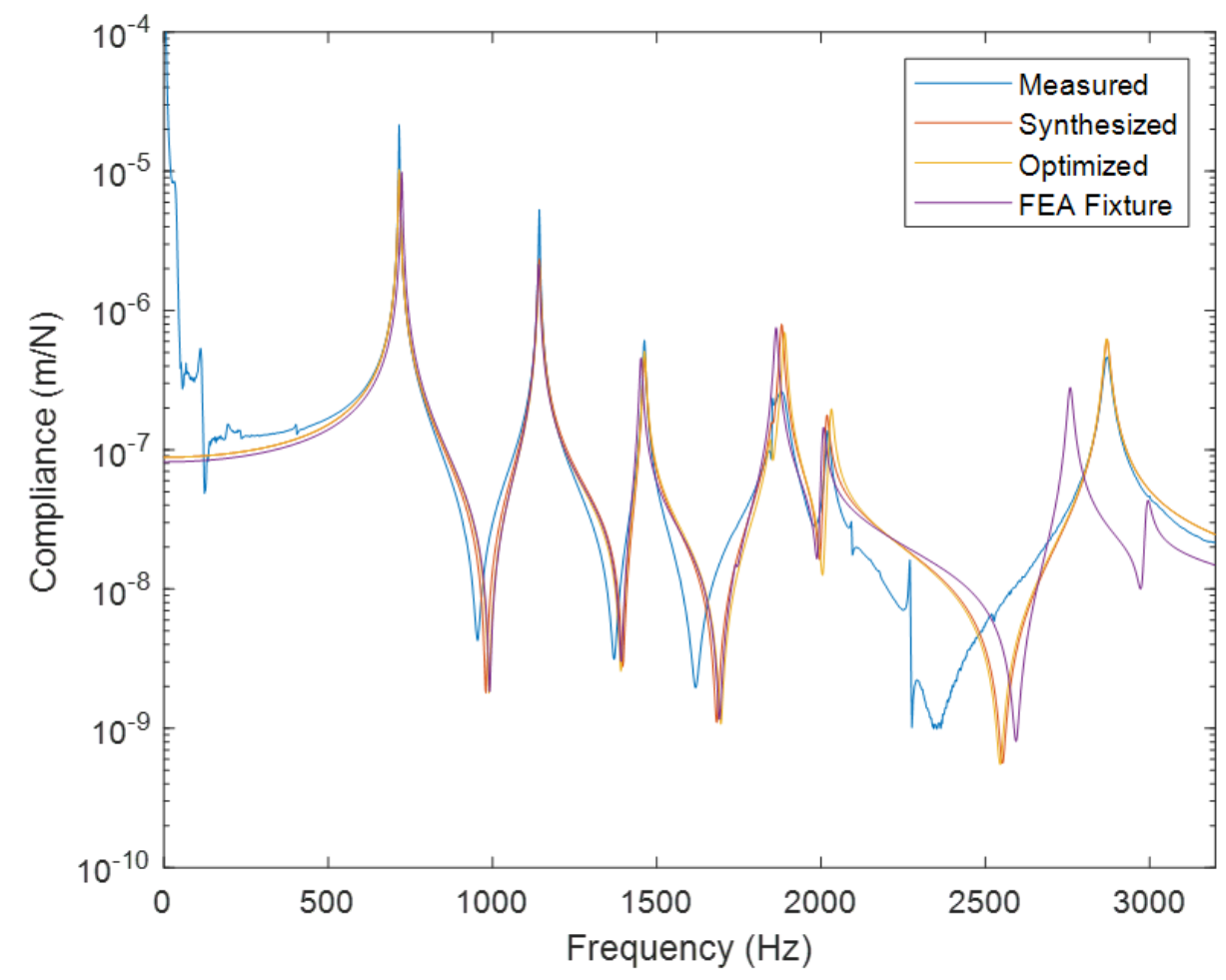

Figure 6.8 Comparison of attachment FRFs between the target, optimized, and FEM.

\subsection{Fixture Validation}

Once the fixture is constructed, we can validate it in the same fashion as the initial fixture design. The box is mounted to the shaker as the surrogate field environment and provided an input profile and the Y-direction response at the attachments is recorded, seen in Figure 6.9. The same test is performed for the LPM fixture design with the same input profile, seen in Figure 6.10. 


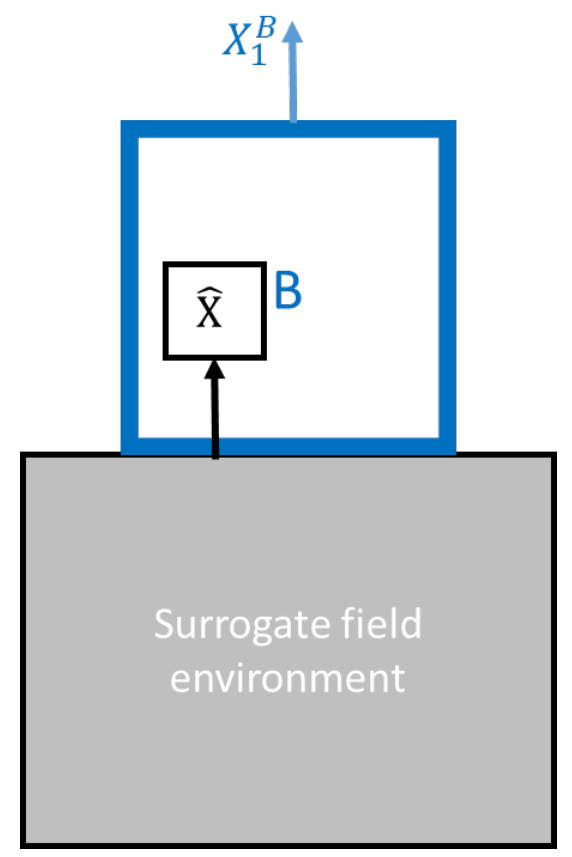

Figure 6.9 Single attachment response recording from shaker excitation of next-structure.

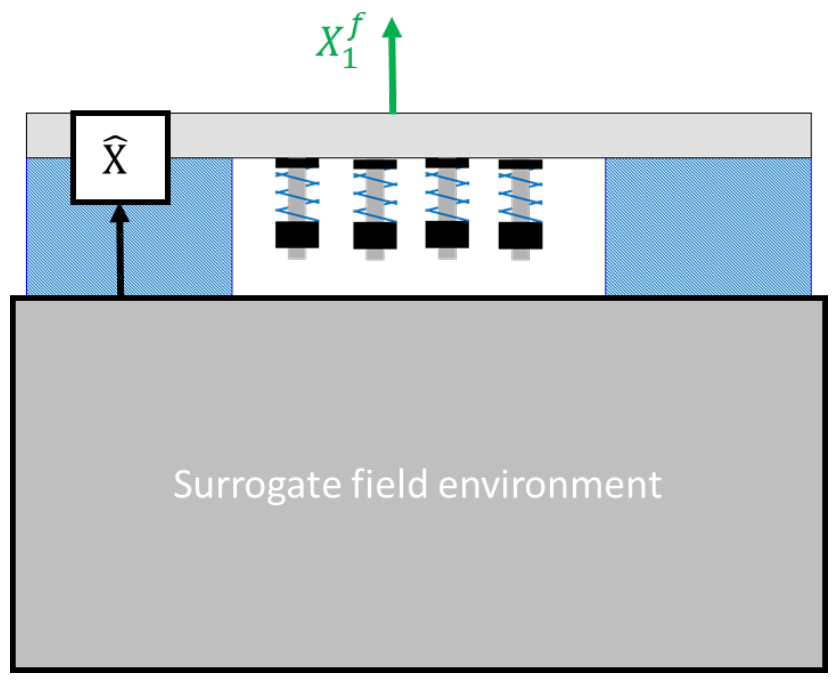

Figure 6.10 Single attachment response recording from shaker excitation of LPM fixture design.

The time history at the attachment location is then used as a control signal for the shaker using waveform replication, seen in Figure 6.11 and Figure 6.12. This test is designed to extract the drive voltage signal to the shaker to allow it to run open loop. This is performed for both the nest-structure and fixture, obtaining two different attachment responses and two different voltage signals. 


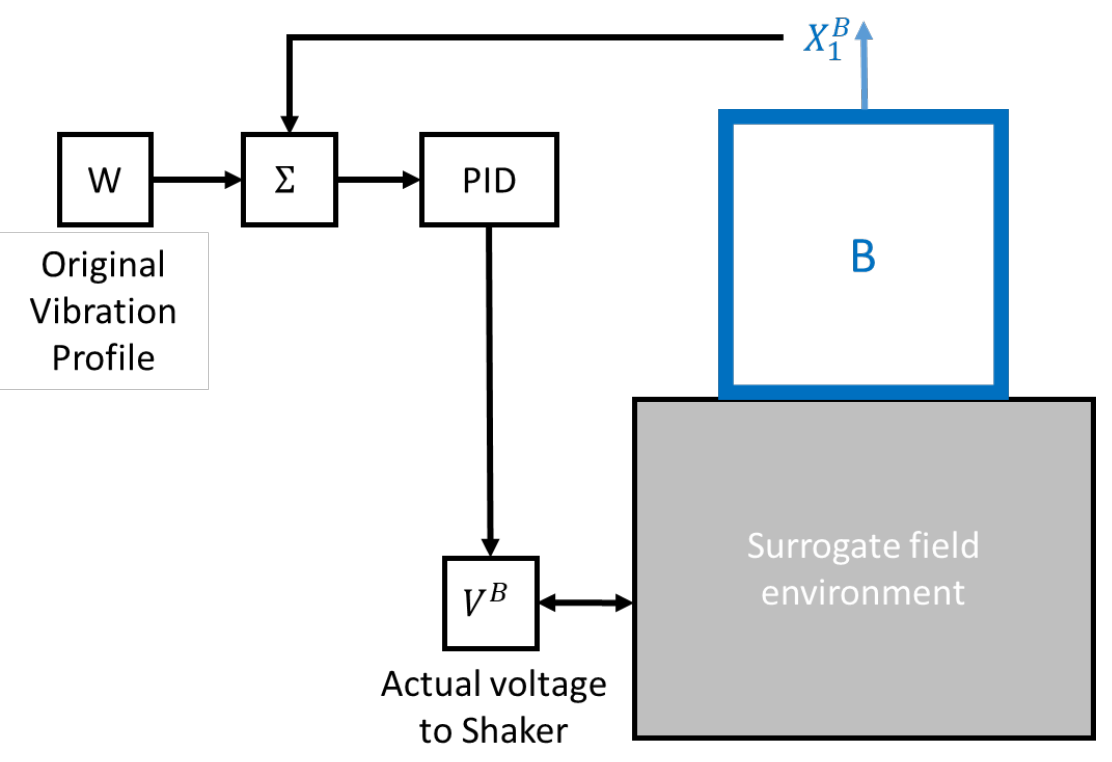

Figure 6.11 Using attachment time history as waveform replicated control shaker input to obtain shaker voltage drive signal for single attachment next-structure.

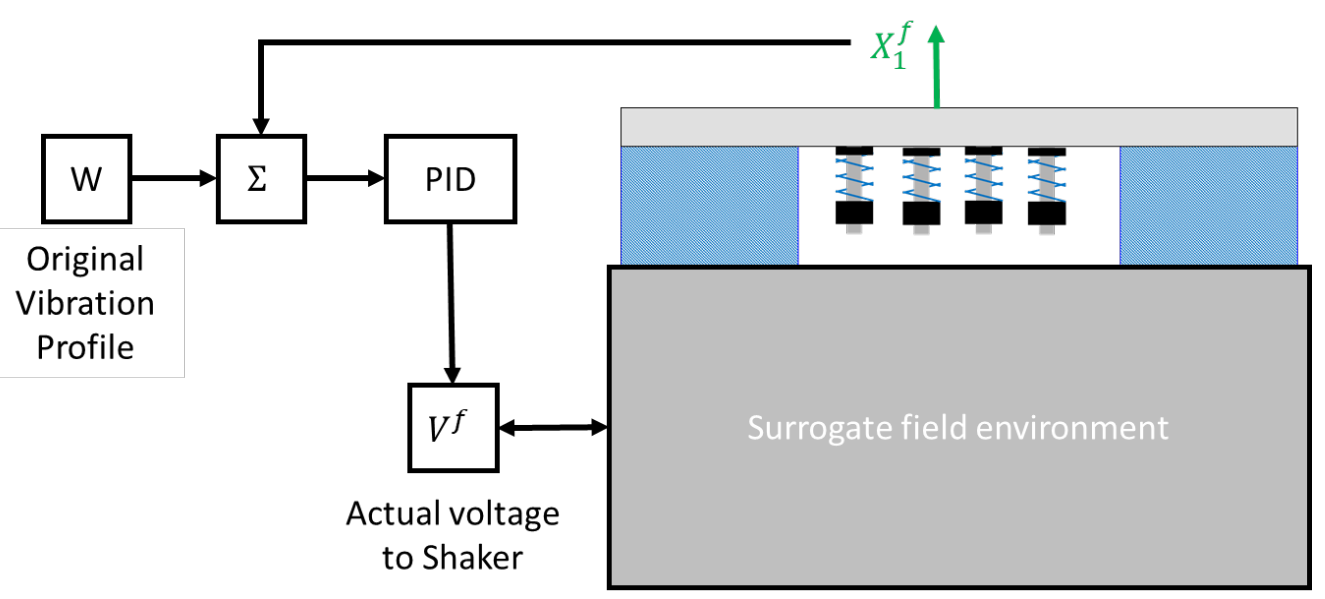

Figure 6.12 Using attachment time history as waveform replicated control shaker input to obtain shaker voltage drive signal for single attachment LPM fixture.

After obtaining the shaker voltage for both the next-structure box and the LPM test fixture, we attach the component to obtain its response and compare. Figure 6.13 and Figure 6.14 show the test set-ups for the box and LPM test fixture, respectively. 




Figure 6.13 Target response collection from voltage drive signal for uncut BARC.

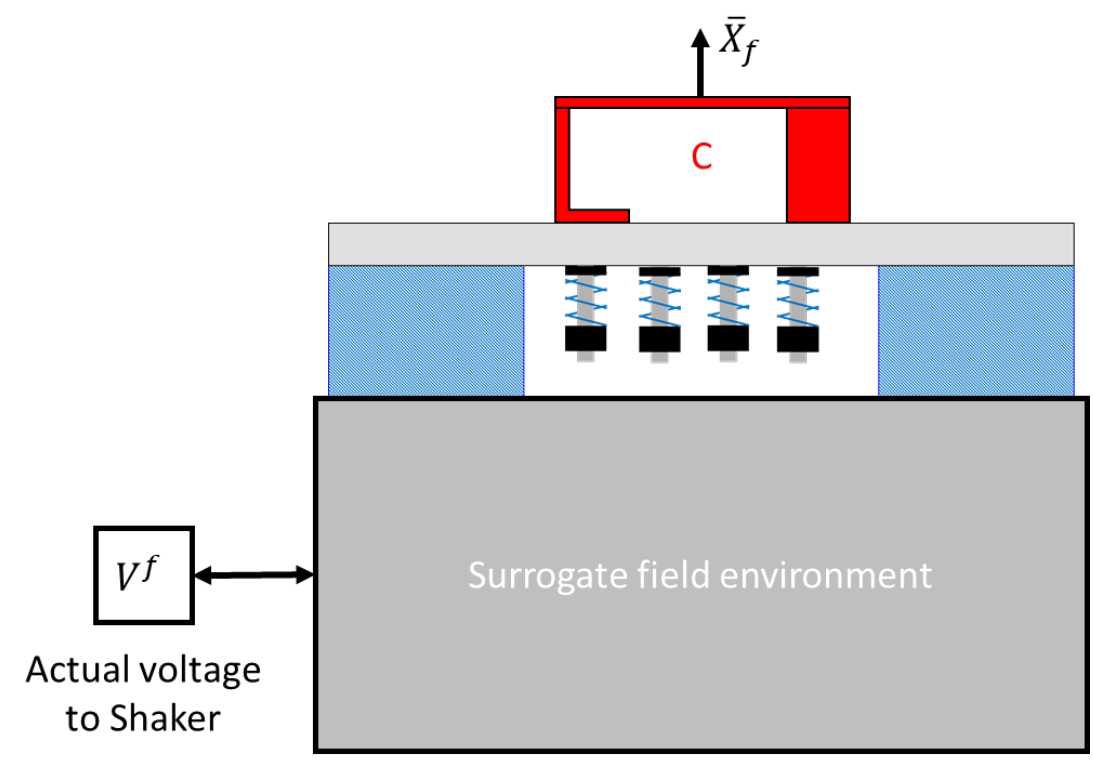

Figure 6.14 Component response on LPM test fixture from voltage drive signal. 


\section{Conclusions and Recommendations}

This chapter discusses the comparison between the designed dynamic test fixture and the next structure Box. Additionally, it discusses the conclusions resolved from the LPM optimization method.

\subsection{Initial Fixture Design}

\subsubsection{Test 1: Attachment FRFs}

I first performed an impact test on the fixture to see if it correlated well with the simulation stiffness characteristics. Figure 7.1 shows the accelerance plots extracted from the measured accelerance of the box (blue), and the fixture (green). The stiffness trends are similar for cross FRFs, but are generally far from the same for diagonal. It is apparent from these results that the stiffness of the fixture likely must increase to achieve a closer representation of the box.

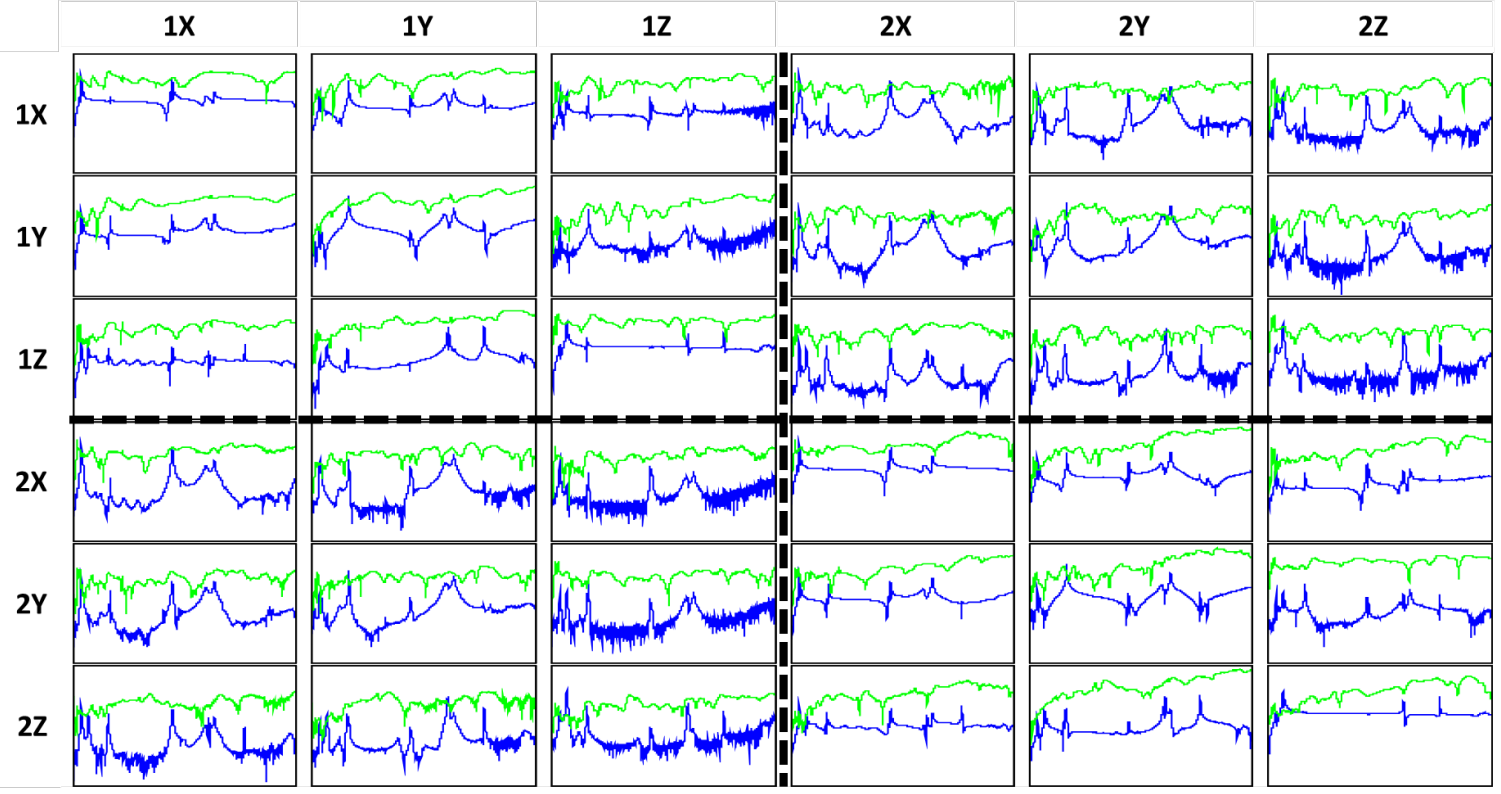

Figure 7.1 Accelerance of the Box (Blue) Fixed and the Fixture (green) Fixed (Y-axis: Log 1e-5-1000 $\mathrm{N} / \mathrm{m} \mathrm{X}$-axis: Linear 0-3200 Hz) 
The figure shows all three directions, but we were only interested in the similarities between the box and the fixture in the Y-direction as that is the direction excited with the shaker. However, the component was designed to have off-axis motion when excited in one axis [1]. This means that even with simplifying to using only the Y-direction for excitation and response there are dynamics that weren't identified.

\subsubsection{Free-free Box vs. Fixture}

The dynamic fixture was originally designed based on the attachment FRFs and mode shapes from the box in a free-free condition. We can compare the time response across the component between the BARC and the fixture to see how successful the fixture is in replicating the response. Figure 7.2 shows the time response comparison at point 1 on the component from the free-free random base input box condition.

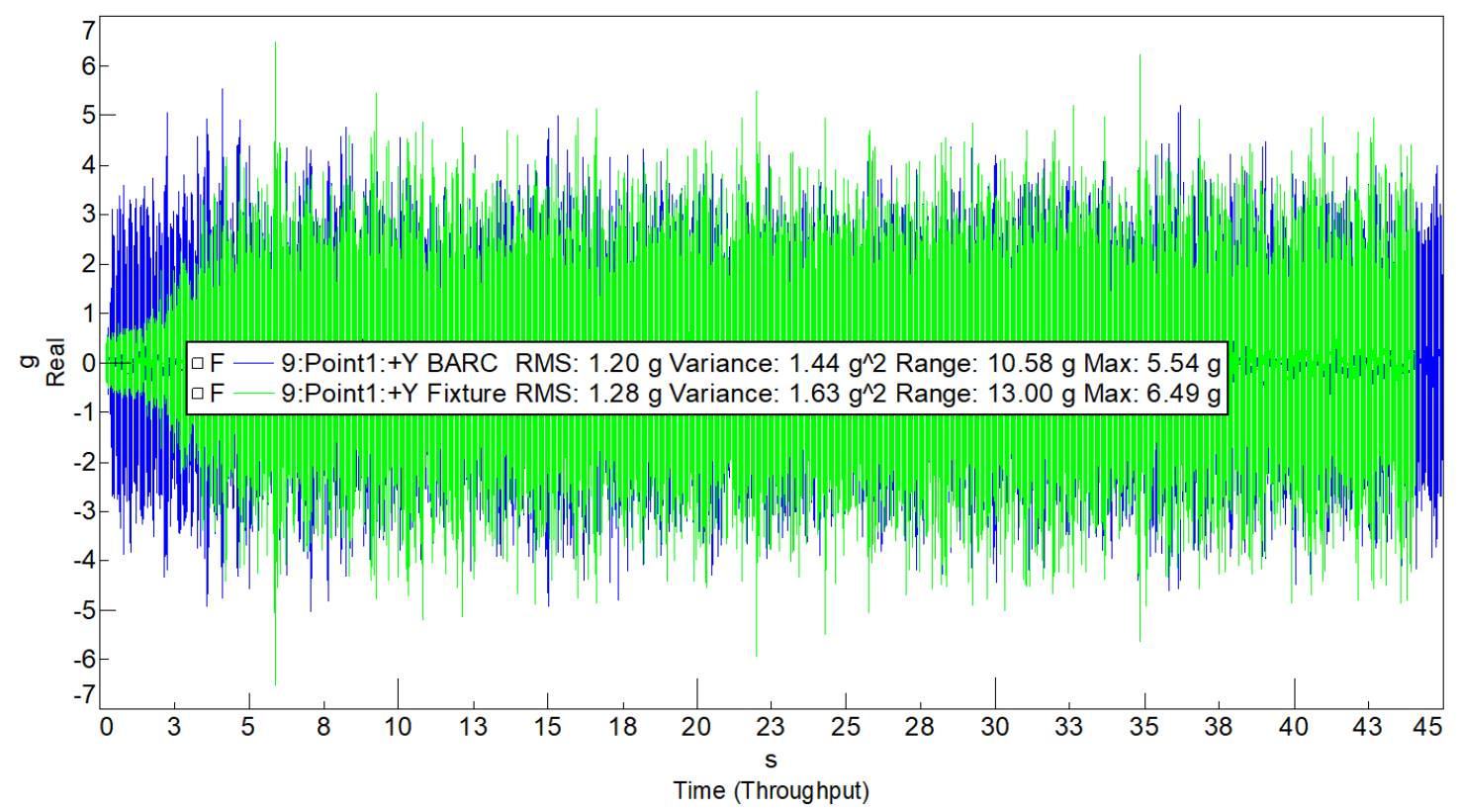

Figure 7.2 Free-free BARC vs. fixture time response at point 1 with RMS, variance, range, and maximum values for each signal.

The response comparisons from points 2-6 can be seen in 9B, but the metrics of comparison can be found in Table 7.1. Overall we see that the response between the two structures is 
relatively similar, however there are some differences as a result of the box being stiffer than the fixture. This can be remedied by using stiffer rubber or metal pillars for the fixture.

Table 7.1 Free-free BARC vs. fixture time response comparison metrics values and differences.

\begin{tabular}{|c|c|c|c|c|c|}
\hline & & $\operatorname{RMS}(g)$ & Var. $\left(g^{2}\right)$ & Range (g) & $\operatorname{Max} .(g)$ \\
\hline \multirow[t]{3}{*}{ Point 1} & BARC & 1.20 & 1.44 & 10.58 & 5.54 \\
\hline & Fixture & 1.28 & 1.63 & 13.00 & 6.49 \\
\hline & $\Delta$ & -0.08 & -0.19 & -2.42 & -0.95 \\
\hline \multirow[t]{3}{*}{ Point 2} & BARC & 1.88 & 3.55 & 16.23 & 8.22 \\
\hline & Fixture & 1.64 & 2.70 & 15.15 & 7.34 \\
\hline & $\Delta$ & 0.24 & 0.85 & 1.08 & 0.88 \\
\hline \multirow[t]{3}{*}{ Point 3} & BARC & 1.56 & 2.45 & 13.72 & 6.41 \\
\hline & Fixture & 2.17 & 4.70 & 19.62 & 9.99 \\
\hline & $\Delta$ & -0.61 & -2.25 & -5.9 & -3.58 \\
\hline \multirow[t]{3}{*}{ Point 4} & BARC & 3.35 & 11.20 & 30.87 & 15.66 \\
\hline & Fixture & 2.62 & 6.84 & 23.75 & 12.29 \\
\hline & $\Delta$ & 0.73 & 4.36 & 7.12 & 3.37 \\
\hline \multirow[t]{3}{*}{ Point 5} & BARC & 2.94 & 8.64 & 26.77 & 13.23 \\
\hline & Fixture & 2.28 & 5.18 & 20.69 & 10.75 \\
\hline & $\Delta$ & 0.66 & 3.46 & 6.08 & 2.48 \\
\hline \multirow[t]{3}{*}{ Point 6} & BARC & 0.96 & 0.94 & 9.50 & 5.28 \\
\hline & Fixture & 1.06 & 1.12 & 10.33 & 4.96 \\
\hline & $\Delta$ & -0.1 & -0.18 & -0.83 & 0.32 \\
\hline
\end{tabular}

\subsubsection{Fixed Box vs. Fixture}

The second validation testing method discussed was based on the box fixed to the shaker. As a result of the fixture being designed based on the free-free box FRFs there are major differences in the component response between the BARC and the test fixture. Figure 7.3 
shows the time histories collected from point 1 on the component on both the fixed BARC and the fixture. The other point responses can be seen in Appendix 9B.

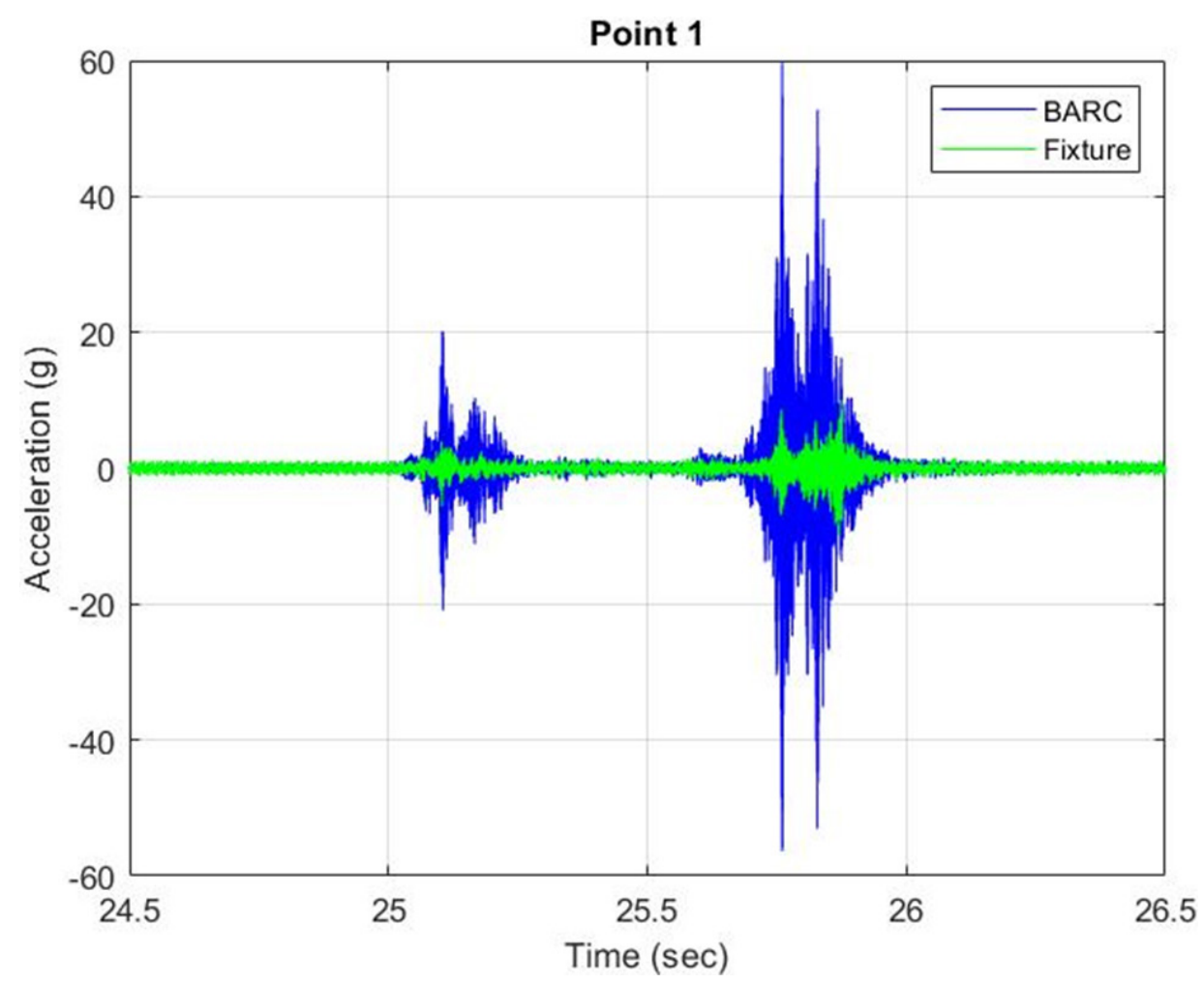

Figure 7.3 Component response comparison on fixture vs. BARC at point 1 with field data input.

We see from the response that the fixture produced a much lower amplitude across the time history. This is common throughout all of the point responses seen in Appendix 9B. The amplitude discrepancy is due to the stiffness of the fixture being less than the box in addition to the box being fixed which adds more stiffness to the structure that wasn't originally accounted for in the fixture design.

Table 7.2 shows some metrics of comparison between the BARC and fixture across all of the points. This table confirms the common amplitude difference between the two structures.

Table 7.2 Fixed BARC vs. Fixture component time response comparison metrics values.

\begin{tabular}{cccccc} 
& & RMS $(\boldsymbol{g})$ & Var. $\left(\boldsymbol{g}^{2}\right)$ & Range $(\boldsymbol{g})$ & Max. $(\boldsymbol{g})$ \\
\hline Point 1 & BARC & 1.17 & 1.38 & 116.00 & 59.66 \\
\hline
\end{tabular}




\begin{tabular}{|c|c|c|c|c|c|}
\hline & Fixture & 0.56 & 0.31 & 20.00 & 10.03 \\
\hline & $\Delta$ & 0.61 & 1.07 & 96 & 49.63 \\
\hline \multirow[t]{3}{*}{ Point 2} & BARC & 1.50 & 2.26 & 146.44 & 70.28 \\
\hline & Fixture & 0.83 & 0.69 & 56.92 & 32.79 \\
\hline & $\Delta$ & 0.67 & 1.57 & 89.52 & 37.49 \\
\hline \multirow[t]{3}{*}{ Point 3} & BARC & 3.23 & 10.41 & 250.80 & 124.87 \\
\hline & Fixture & 1.34 & 1.79 & 124.97 & 66.43 \\
\hline & $\Delta$ & 1.89 & 8.62 & 125.83 & 58.44 \\
\hline \multirow[t]{3}{*}{ Point 4} & BARC & 2.05 & 4.21 & 139.45 & 72.55 \\
\hline & Fixture & 0.97 & 0.94 & 85.62 & 40.20 \\
\hline & $\Delta$ & 1.08 & 3.27 & 53.83 & 32.35 \\
\hline \multirow[t]{3}{*}{ Point 5} & BARC & 2.38 & 5.68 & 194.69 & 97.07 \\
\hline & Fixture & 0.65 & 0.43 & 43.25 & 23.43 \\
\hline & $\Delta$ & 1.73 & 5.25 & 151.44 & 73.64 \\
\hline \multirow[t]{3}{*}{ Point 6} & BARC & 0.91 & 0.83 & 79.41 & 40.34 \\
\hline & Fixture & 0.41 & 0.17 & 31.28 & 14.90 \\
\hline & $\Delta$ & 0.5 & 0.66 & 48.13 & 25.44 \\
\hline
\end{tabular}

Since the rocket field data shows several instances of pyroshock we were also able to compare the response of the fixture to the BARC using the SRS. Figure 7.4 Fixed BARC vs. fixture SRS from point 1 on component for 3 identified shock events. shows the SRS calculated from point 1 for the three shock events identified from the time response. In these SRS we see that the overall amplitude from the fixture response is lower than that of the fixed BARC. Again, this is due to the stiffness of the BARC being significantly higher than the fixture stiffness. 

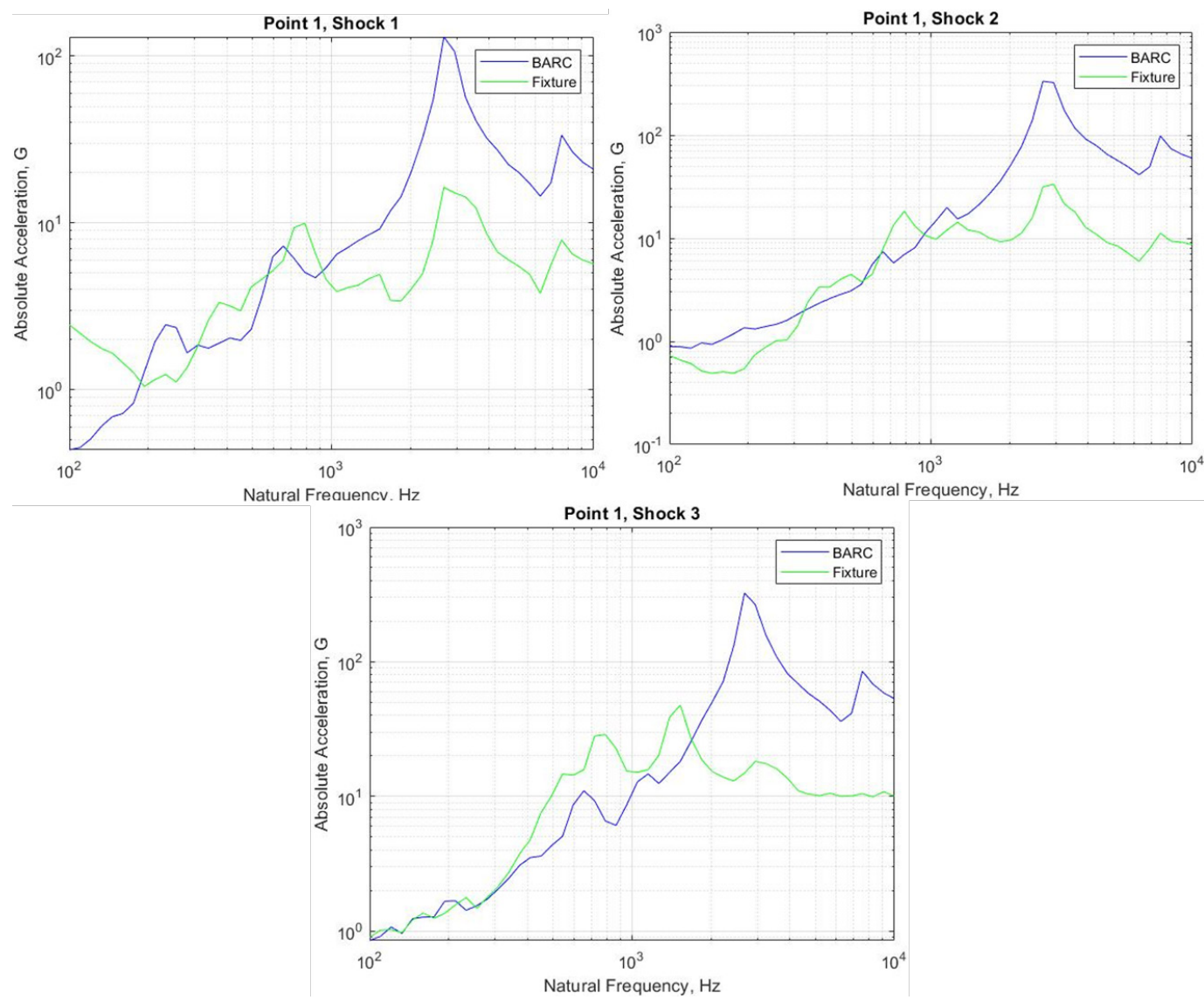

Figure 7.4 Fixed BARC vs. fixture SRS from point 1 on component for 3 identified shock events.

\subsubsection{Recommendations}

We have identified the major contributor between the fixture and BARC response to be the relative stiffness. Although the fixture was designed to have similar directional stiffness to the box, the physical structure did not produce the desired values. This stiffness discrepancy could be a result of the rubber pillars not being rigid enough, the joints connecting the components of the fixture allowing for some rotation and translation, and/or the material used for the fixture brackets not producing the stiffness expected due to it being $3 \mathrm{D}$ printed.

This fixture appears to be a viable option in replicating the BARC response, however adjustments would have to be made if we wished to continue with this design. I would 
suggest we use a material for the legs of the fixture that is stiffer than the $90 \mathrm{~A}$ rubber we used. I would also suggest gluing the joints holding the fixture together to limit any overall stiffness reducing motion. Finally, 3D printing materials results in a structure that has gaps present inside of it, therefore machining the fixture brackets out of a stiffer material would reduce this and could result in a large increase of overall stiffness.

The test fixture designed and analyzed in this work successfully produced the relative motion between the attachments of the component necessary to the boundary condition challenge problem. Although the test fixture is a physical step toward solving this problem, it is not sufficient. Additional dynamics and stiffness could be used to drive the natural frequencies and mode shapes closer to that of the next-structure. From this work we learned that a physical fixture is realizable. It helped us learn that in using knowledge of the nextstructure motion and stiffness, it is possible to design a fixture to replicate the dynamics.

\subsection{Parameter Optimization Method}

With this method it may be possible to produce a dynamic fixture that provides the proper excitation at the component attachment locations in a single direction. The fixture for the single attachment next-structure has not been built yet, so we have not yet determined if this method works in the physical world. At this point, however it seems to be a viable solution to the boundary condition challenge problem. Using the initial single attachment model validation of this method we were able to determine a process for fixture design that could be used for next-structures of multiple attachments. The steps for this process are as follows:

1. Measure drive point attachment FRFs.

2. Estimate mass vector $(\mathrm{kg})$ and stiffness $(\mathrm{kN} / \mathrm{mm})$ modal parameters

3. Make a LPM mass and stiffness model

4. Optimize to match modal parameters

5. Design the physical system

6. Verify attachment FRFs 
We have already worked through the first three steps of this method for a dual attachment next-structure. Figure 7.5 shows the test set-up for obtaining the attachment FRFs.

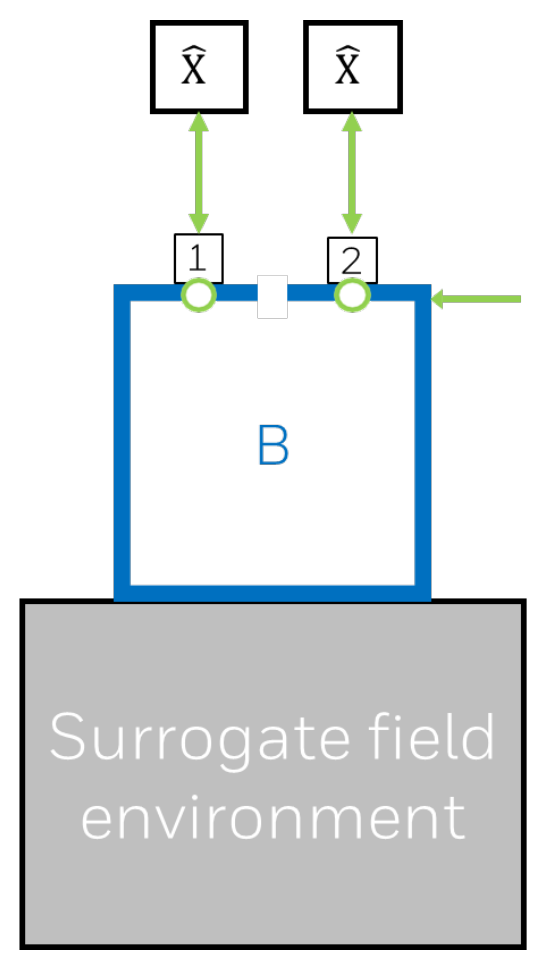

Figure 7.5 Impact test set-up used to gather the attachment FRFs from the next-structure in its surrogate field environment.

The method would be the same as the single attachment, except the LPM would require two masses or a very flexible main beam to allow for relative dynamics between the attachment locations, seen in Figure 7.6. In this model $H_{22}^{f}$ and $H_{11}^{f}$ are the attachment FRFs from each of the two attachment locations. 


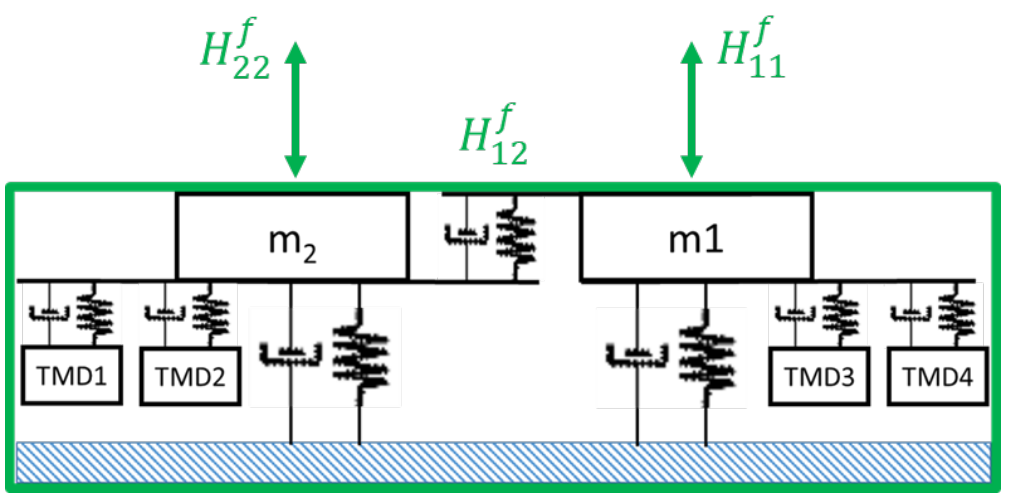

Figure 7.6 Lumped Parameter Model of next-structure, used for optimization of parameters to match attachment FRFs.

The dual attachment fixture would be able to represent the true BARC structure. Figure 7.7 shows the measured vs. synthesized attachment FRFs from the box next-structure from the curve-fitting process.
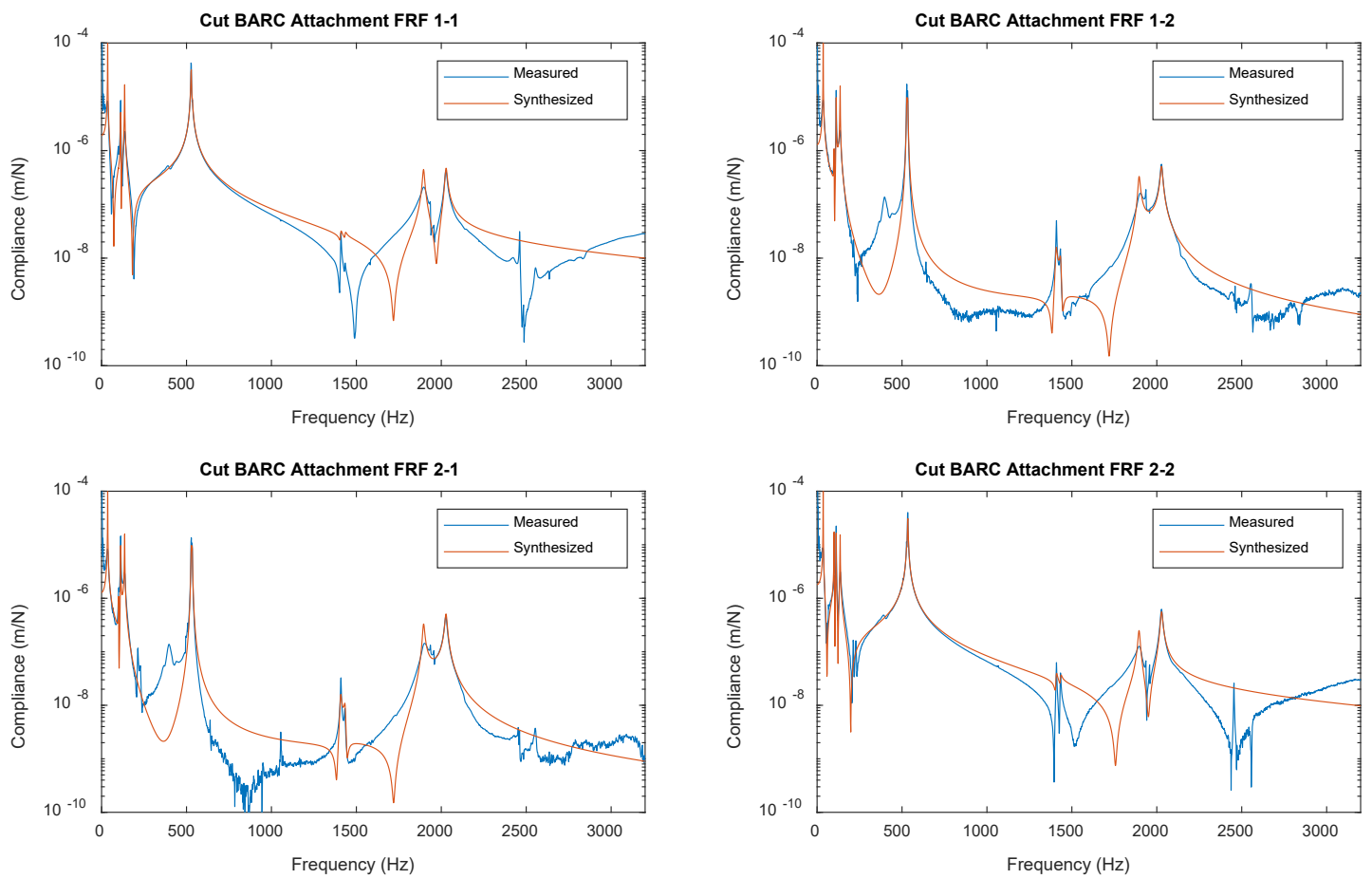

Figure 7.7 Measured vs. synthesized attachment FRFs from the cut box of the BARC structure. 
After running the optimization, the answer did not converge to a solution after several iterations. It is possible that the initial values were too far off for the optimization to converge quickly, but more research needs to be expended to improve the optimization. In the interest of the $\mathrm{BC}$ challenge originally defined, with this we could have a dynamic testfixture that produces the field response and limits over and undertesting dramatically. 


\section{Future Work}

Tuning of the initial fixture design could be a viable option in replicating the attachment response and therefore the $\mathrm{BCs}$ experiences unto the component. I suggest a next step would be to add stiffness to the structure through different material for the pillars and/or brackets. Once the overall stiffness of the structure resembles that of the box, tuning by means of TMDs or design changes could improve the response.

With respect to the optimized LPM method, I would first like to build a physical structure based on the fixed-fixed beam with TMDs LPM design, and perform the validation tests described. Also, adding to the optimization code to see how the component response differs as a result of the slight difference between the LPM fixture and next-structure FRFs. Once we determine if the single attachment is viable through the physical testing, the exploration of a multiple attachment next-structure should be continued. The dual attachment nextstructure optimization should also be explored for improvement in the convergence upon a solution.

Additionally, there is currently no explicitly defined metric for "success". How close to the next-level assembly field response do we really have to be to be successful? The environmental testing community should better define this "success". Strain is often associated with over and undertesting and expected life and could be a possible metric to be defined as the goal of the boundary condition challenge results. SRS are also used as standards often times and could be used to define the success of a fixture, however it is possible to produce the exact same SRS with dynamically different structures.

I would like more analysis of the response gathered from the component on the original fixture to be done to better define its "success". Power Spectral Density of the random vibration response, better formulated SRS calculations, and strain response are three of the analysis I would like to perform on the component in its next-level assembly and fixture. This should be done for both methods, the initial fixture design and the optimized lumped parameter method (both single and dual attachment). This analysis should allow us to better 
understand the true nature of our fixture dynamics and how they impact the component response, and maybe help define a standard of success in this challenge.

Finally, once a method is developed it may be lucrative to design fixture construction kits for various size structures. These kits could include sets masses, springs, and dampers that are designed for different sizes and masses of next-structures. With this kit one could perform the analysis as defined by the optimized LPM method and then easily build a tunable dynamic test fixture. 


\section{Reference List}

[1] R. J. J. David E. Soine, Jr, "Designing Hardware for the Boundary Condition Round Robin Challenge," presented at the International Modal Analysis Conference XXVI, Orlando, FL, 2018.

[2] P. C. Tyler F. Schoenherr, Brett Clark, "Use of Topology Optimization to Design Shock and Vibration Test Fixtures," presented at the International Modal Analysis Conference XXXVII, Orlando, FL, 2019.

[3] N. F. Hunter, "Vibration Testinf Reviewing the State of the Art-Prospects and Challenges," in SD 2000 Conference, 2000.

[4] D. R. a. D. M. Blackburn, "The History of a Decision: A Standard Vibration Test Method for Qualification," 2017.

[5] "What is transfer path analysis," S. P. Software, Ed., ed, 2014.

[6] C. VanKarsen, "Digital Signal Processing Frequency Domain Measurements PSD," ed, 2018.

[7] "What is a Power Spectral Density (PSD)?," Community Article,

[8] J. E. Alexander, "Shock Response Spectrum - A Primer," Sound And Vibration, 2009.

[9] P. Avitabile, Modal Testing A Practitioner's Guide. Society of Experimental Mechanics and John Whiley \& Sons Ltd, 2018.

[10] E. M. Jacobson, "Using Frequency Based Substructuring to Optimize Multi-Axis Resonant Plate Shock Tests," M.S. Mechanical Engineering, Mechanical Engineering - Engineering Mechanics, Michigan Technological University, 2019.

[11] N. Jalili, "An Introduction to Vibrations of Lumped-Parameter Systems," in Piezoelectric-Based Vibration ControlNew York, NY: Springer Science+Business Media LLC, 2010.

[12] D. J. R. D. de Klerck, S.N. Voormeeren, "General Framework for Dynamic Substructuring: History, Review, and Classification of Techniques," AIAA Journal, vol. 46 , no. $5,2008$.

[13] J. R. B. Erica M. Jacobson, James P. DeClerck, Charles D. Van Karsen, David Soine, "Frequency Based Substructuring on a Resonant Plate," in International Modal Analysis Conference XXXVIII, Houston, TX, 2020. 
[14] P. A. Jesus M. Reyes, Richard Jones, David Soine, "Fixture Neutralization Method - Adjustment of Vibration Response to Account for Fixture-Test Arcticle Dynamic Coupling Effects Using Measured Frequency Response Functions," presented at the International Modal Analysis Conference XXXVII, Orlando, FL, 2019.

[15] J. M. Harvie, "Using Modal Substructuring to Improve Shock \& Vibration Qualification," presented at the International Modal Analysis Conference XXXVI, Orlando, FL, 2018.

[16] S. S. Daniel P. Rohe, Matthew R. W. Brake, James DeClerck, Mariano Alvarez Blanco, Tyler F. Schoenherr, Troy Skousen, "Testing Summary for the Box Assembly with Removable Component Structure," presented at the International Modal Analysis Conference XXXVII, Orlando, FL, 2019.

[17] T. F. Schoenherr, "Designing an Optimized Fixture for the BARC Hardware Using a Parameterized Model," presented at the International Modal Analysis Conference XXXVIII, Orlando, FL, 2020.

[18] T. M. Hall, "Analytically Investigating Impedance-Matching Test Fixtures," presented at the International Modal Analysis Conference XXVII, Orlando, FL, 2019.

[19] R. A. S. Daniel P. Rohe, Tyler F. Schoenherr, Troy J. Skousen, "Comparison of Multi-Axis Testing of BARC Structure with varying Boundary Conditions," presented at the International Modal Analysis Conference XXXVII, Orlando, FL, 2019.

[20] K. N. a. M. Schmidt-Ladin, "Proposed 12-DOF Shaker Control of BARC Structure," presented at the International Modal Analysis Conference XXXVIII, Houston, TX, 2020. 


\section{A Box and Fixture FEM Results}

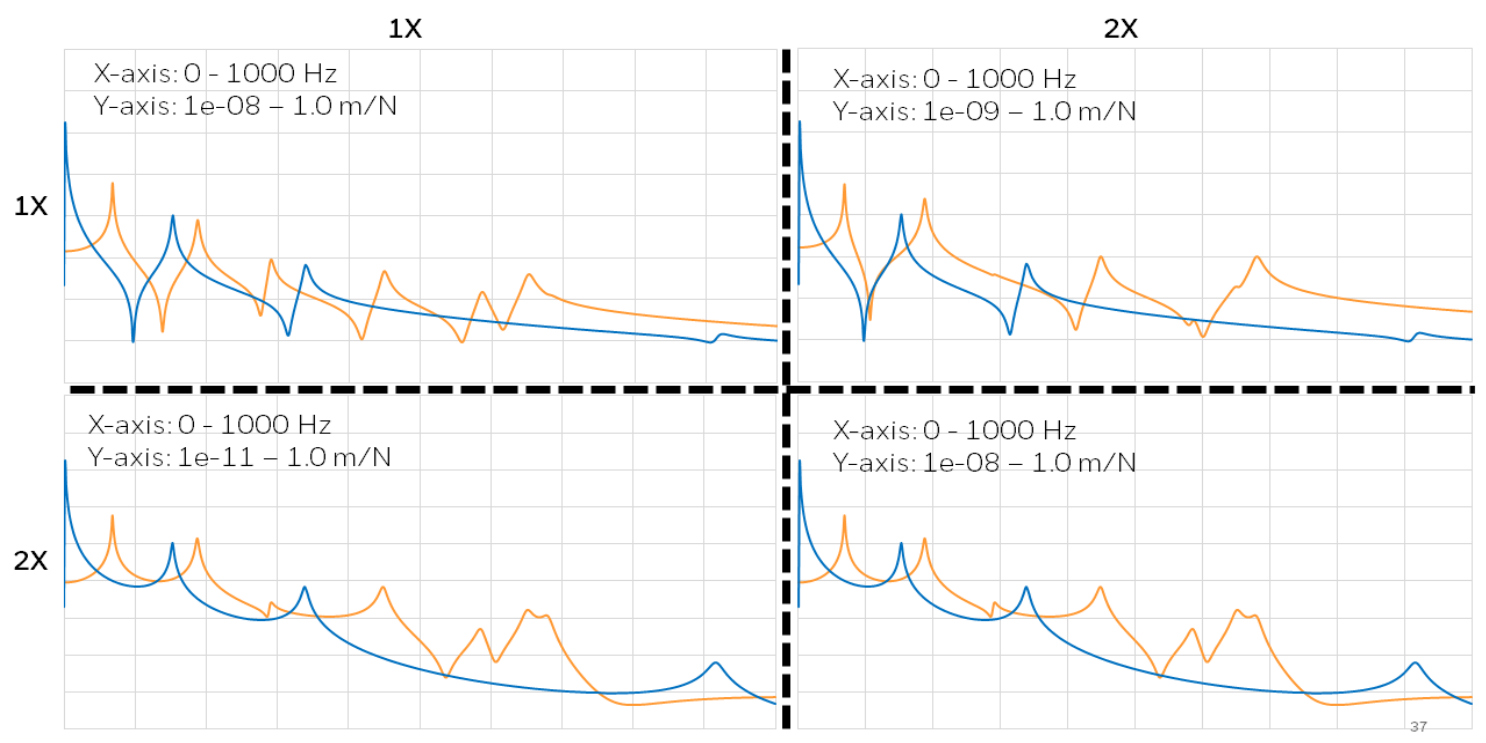

Figure 9.1 X-directional Accelerance Plots from the Fixture (Orange) and Box (Blue).



Figure 9.2 Z-directional Accelerance Plots from the Fixture (Orange) and Box (Blue). 


\section{B BARC vs. Fixture Time Histories}

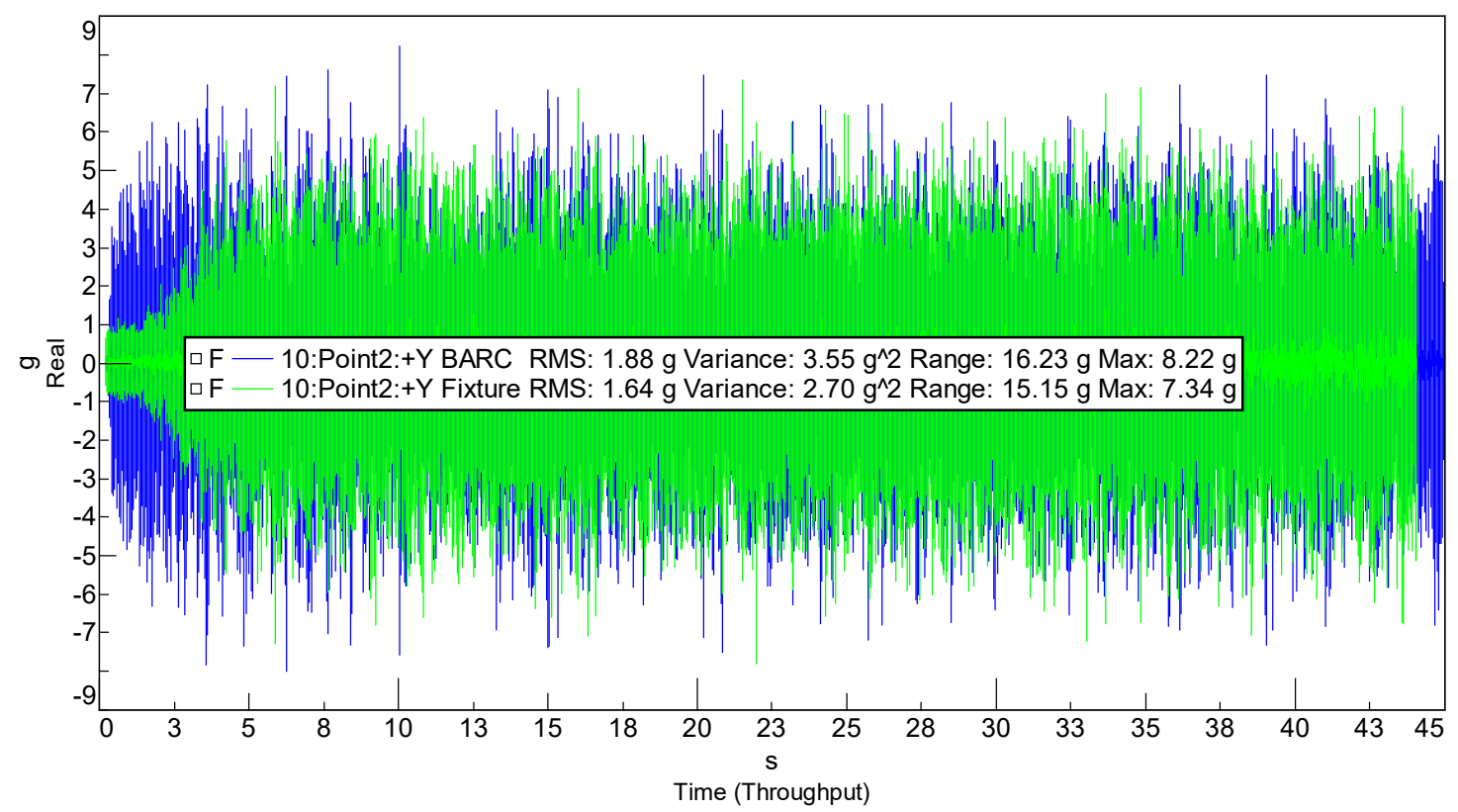

Figure 9.3 Free-free BARC vs. fixture time response at point 2 with RMS, variance, range, and maximum values for each signal.

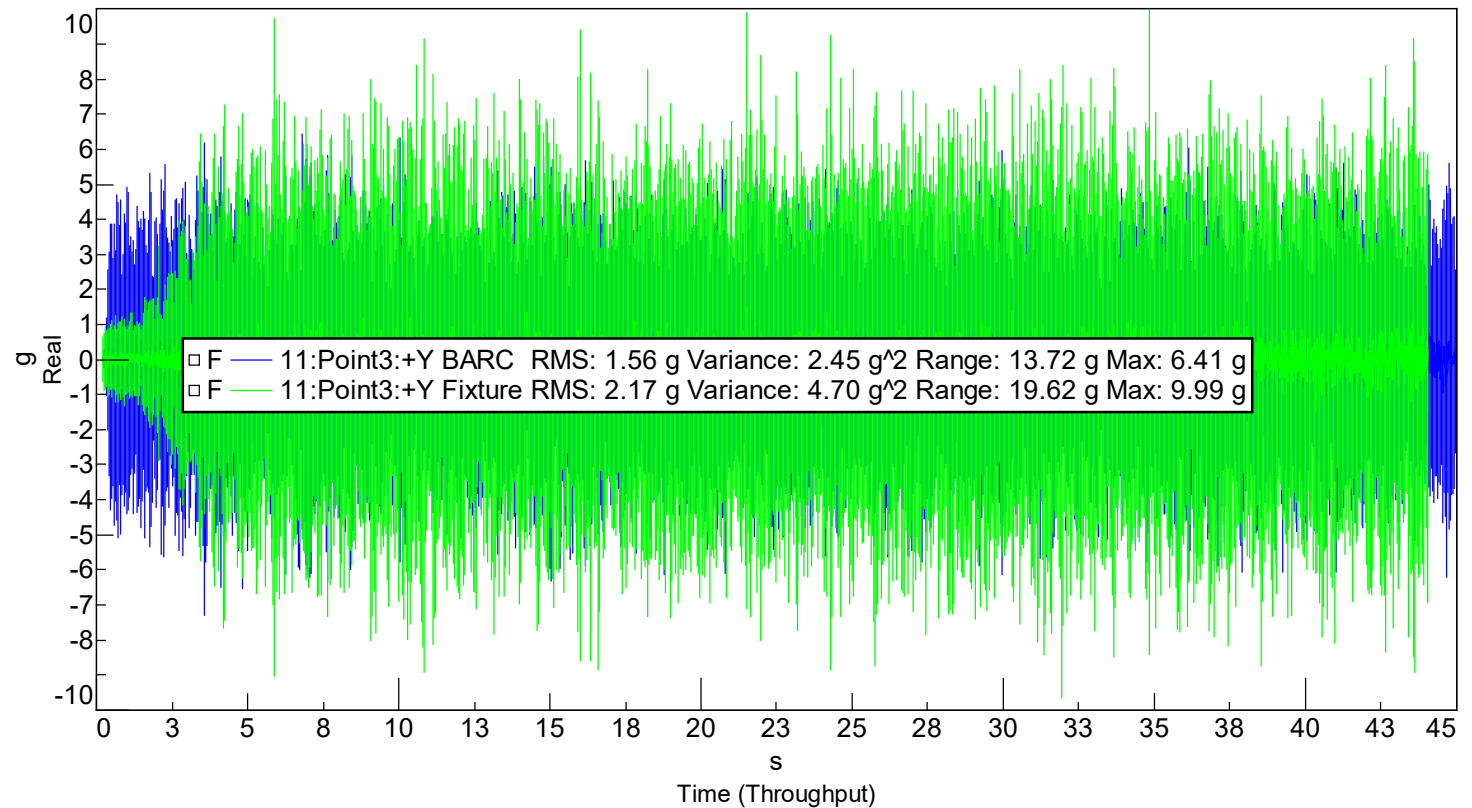

Figure 9.4 Free-free BARC vs. fixture time response at point 3 with RMS, variance, range, and maximum values for each signal. 


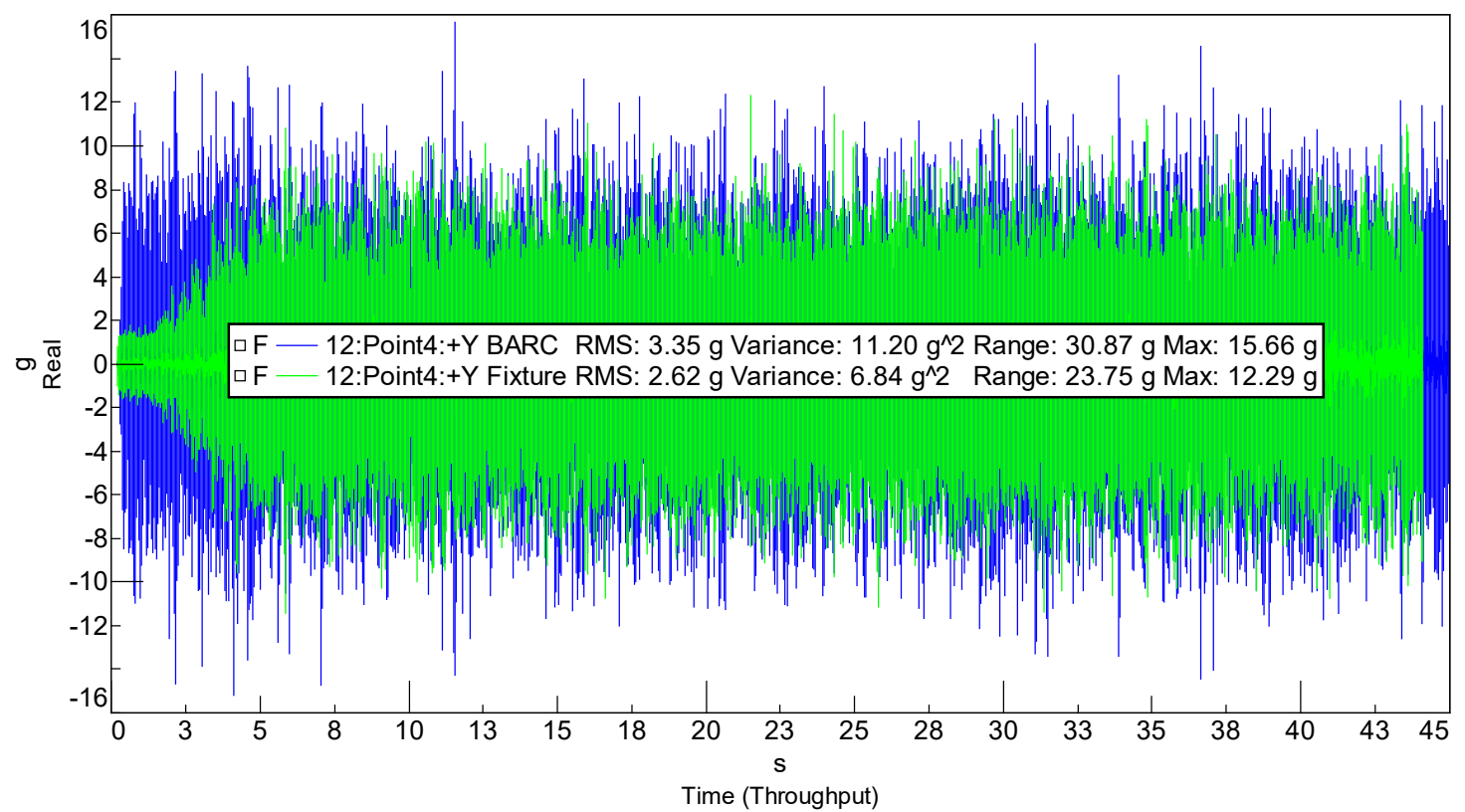

Figure 9.5 Free-free BARC vs. fixture time response at point 4 with RMS, variance, range, and maximum values for each signal.

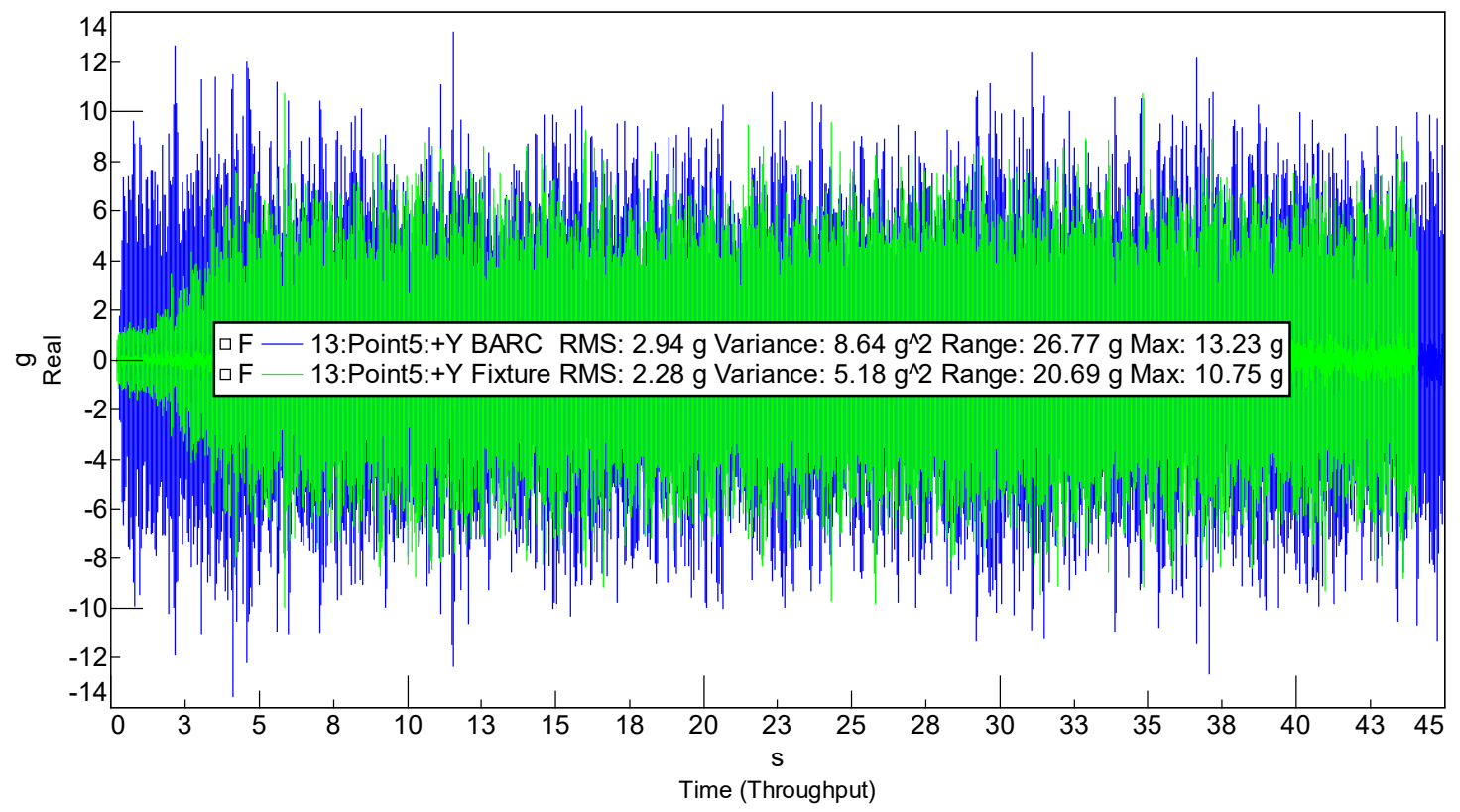

Figure 9.6 Free-free BARC vs. fixture time response at point 5 with RMS, variance, range, and maximum values for each signal. 




Figure 9.7 Free-free BARC vs. fixture time response at point 6 with RMS, variance, range, and maximum values for each signal. 


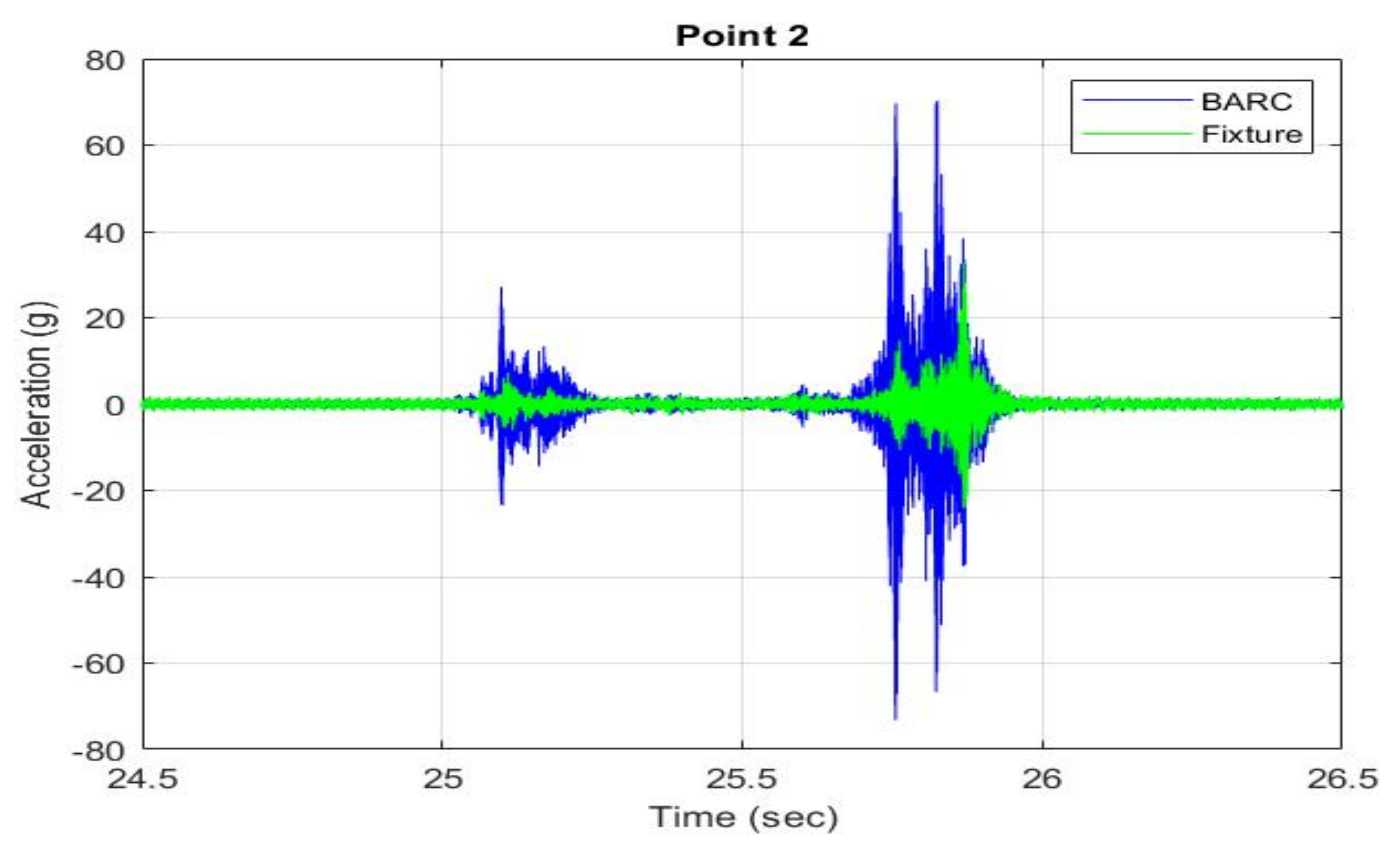

Figure 9.8 Component response comparison on fixture vs. BARC at point 2 with field data input.
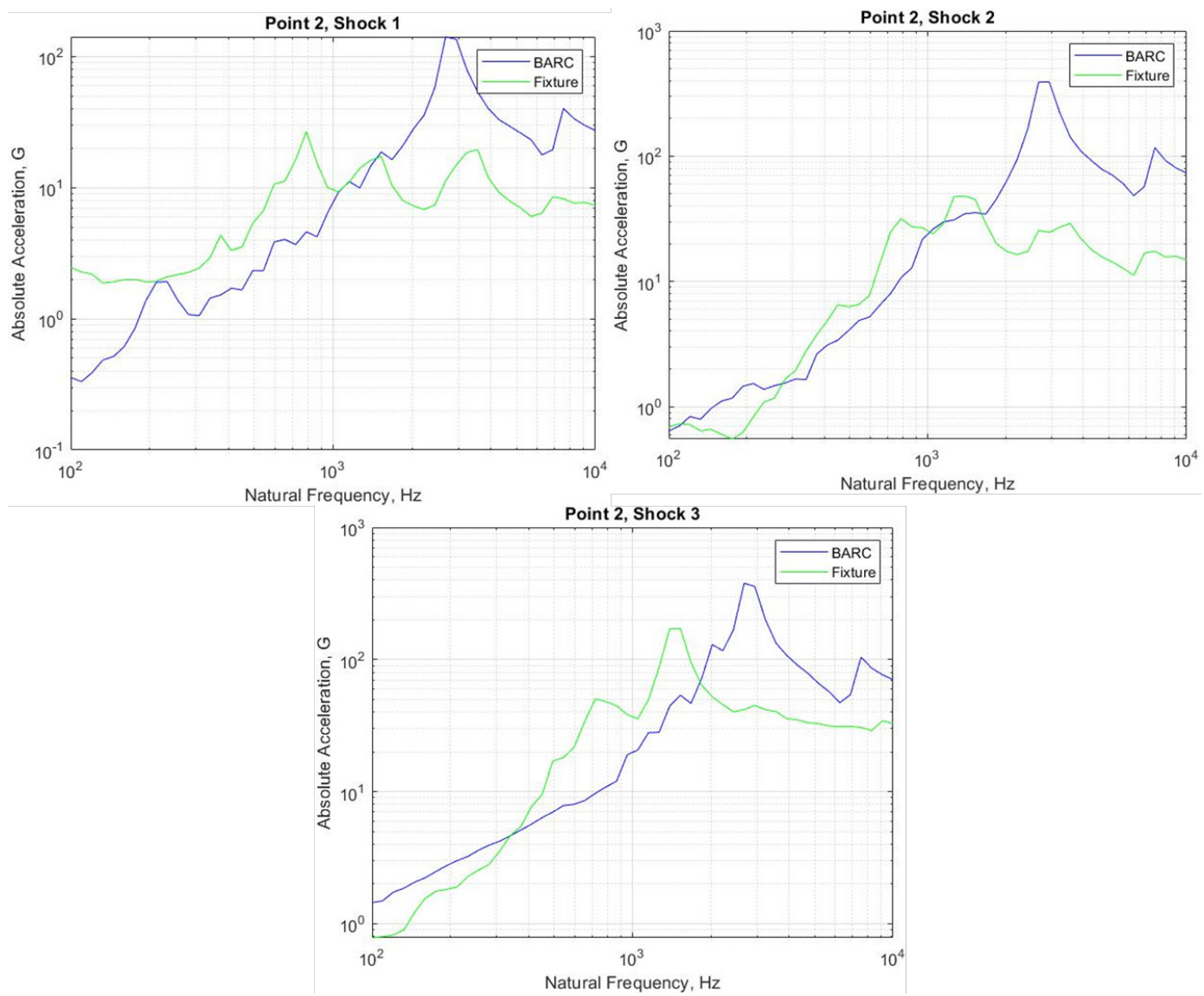

Figure 9.9 Fixed BARC vs. fixture SRS from point 2 on component for 3 identified shock events. 




Figure 9.10 Component response comparison on fixture vs. BARC at point 3 with field data input.


Figure 9.11 Fixed BARC vs. fixture SRS from point 3 on component for 3 identified shock events. 


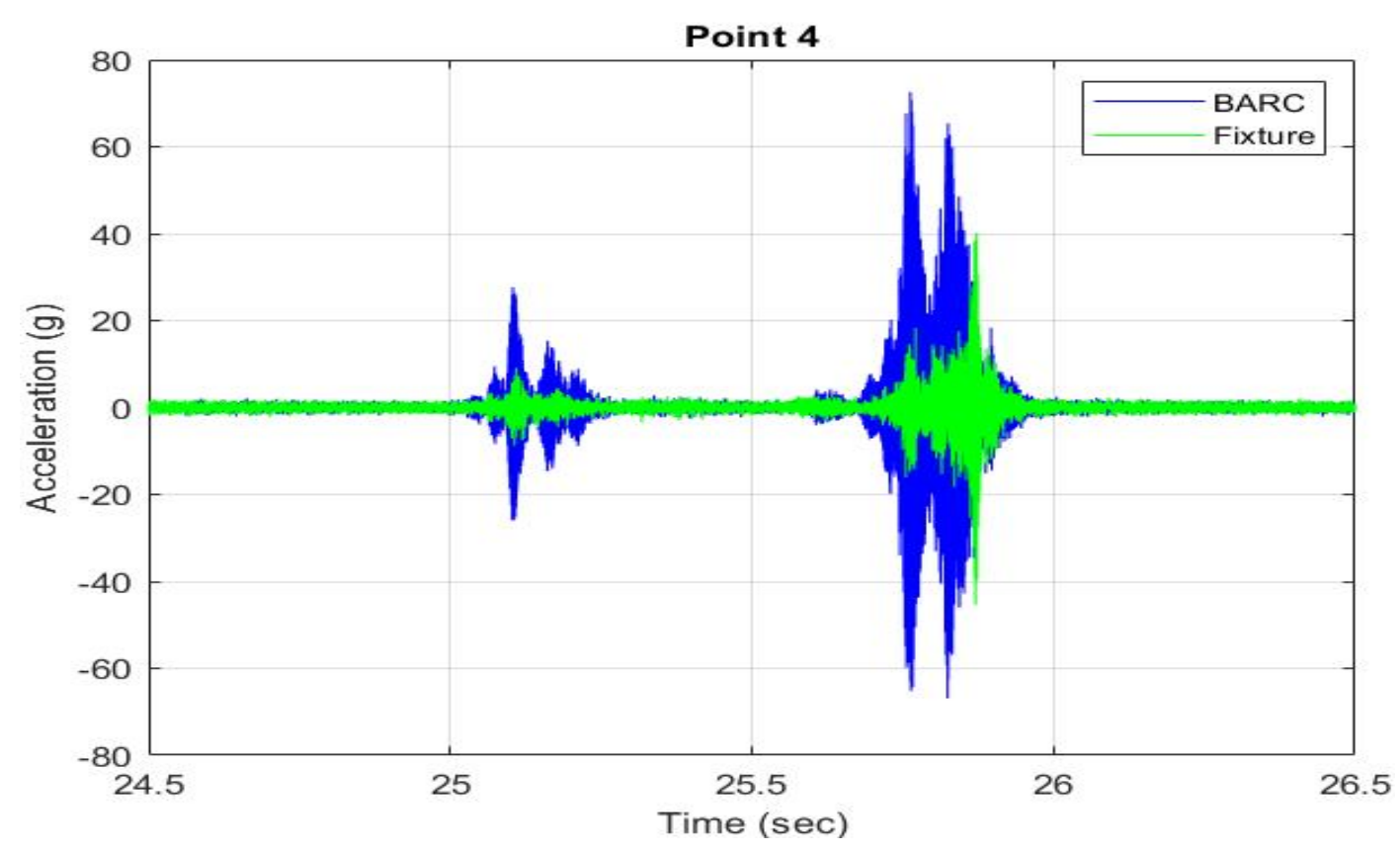

Figure 9.12 Component response comparison on fixture vs. BARC at point 4 with field data input.
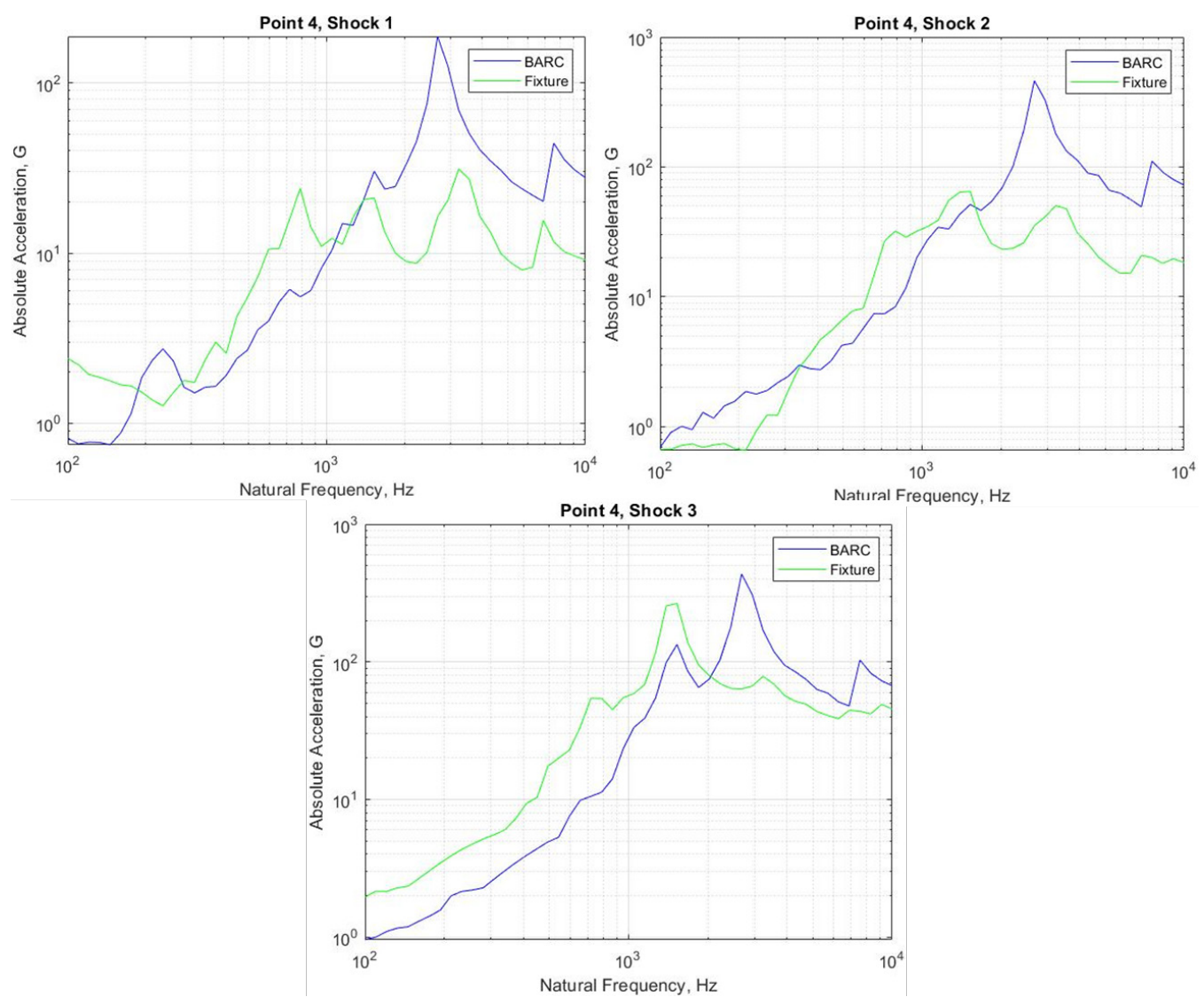

Figure 9.13 Fixed BARC vs. fixture SRS from point 4 on component for 3 identified shock events. 


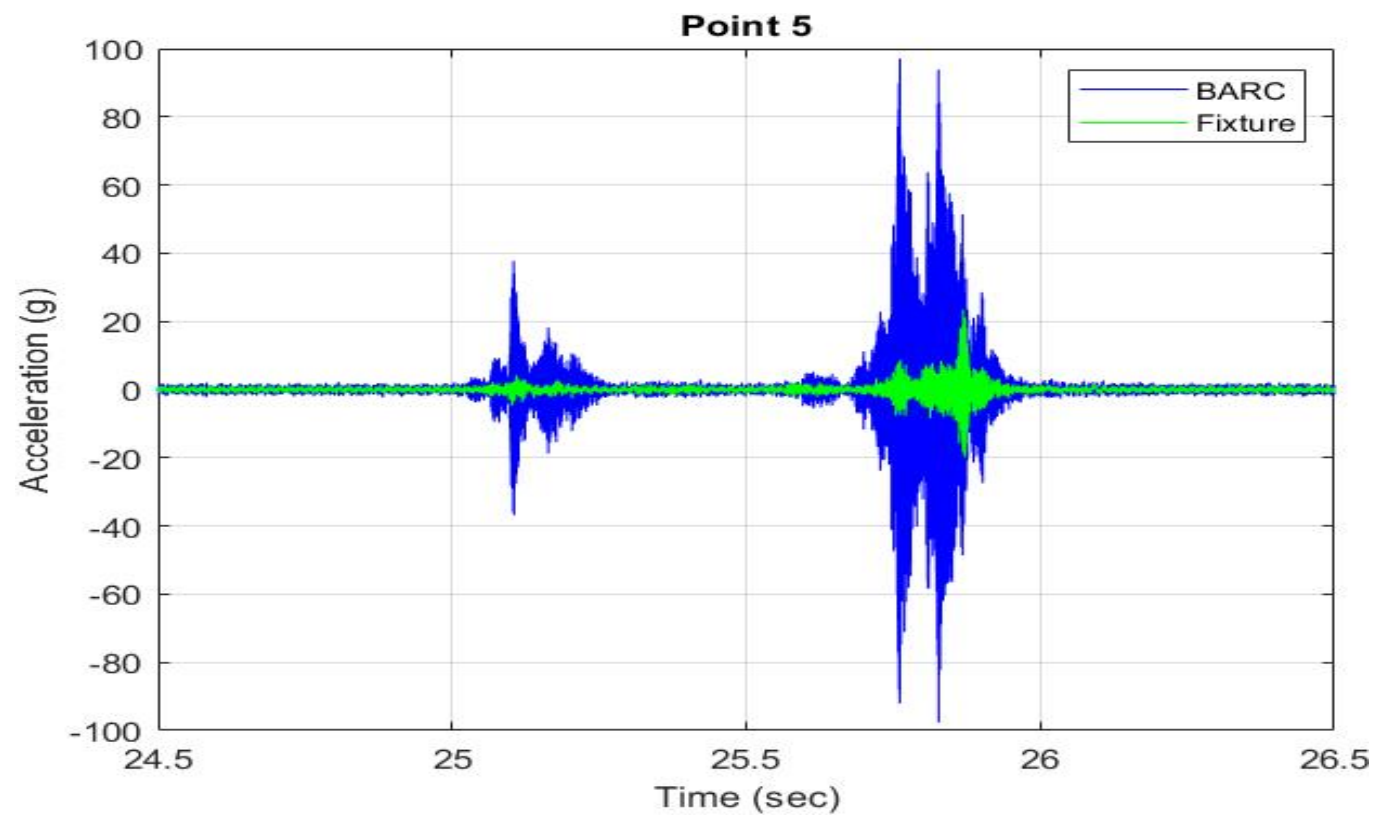

Figure 9.14 Component response comparison on fixture vs. BARC at point 5 with field data input.
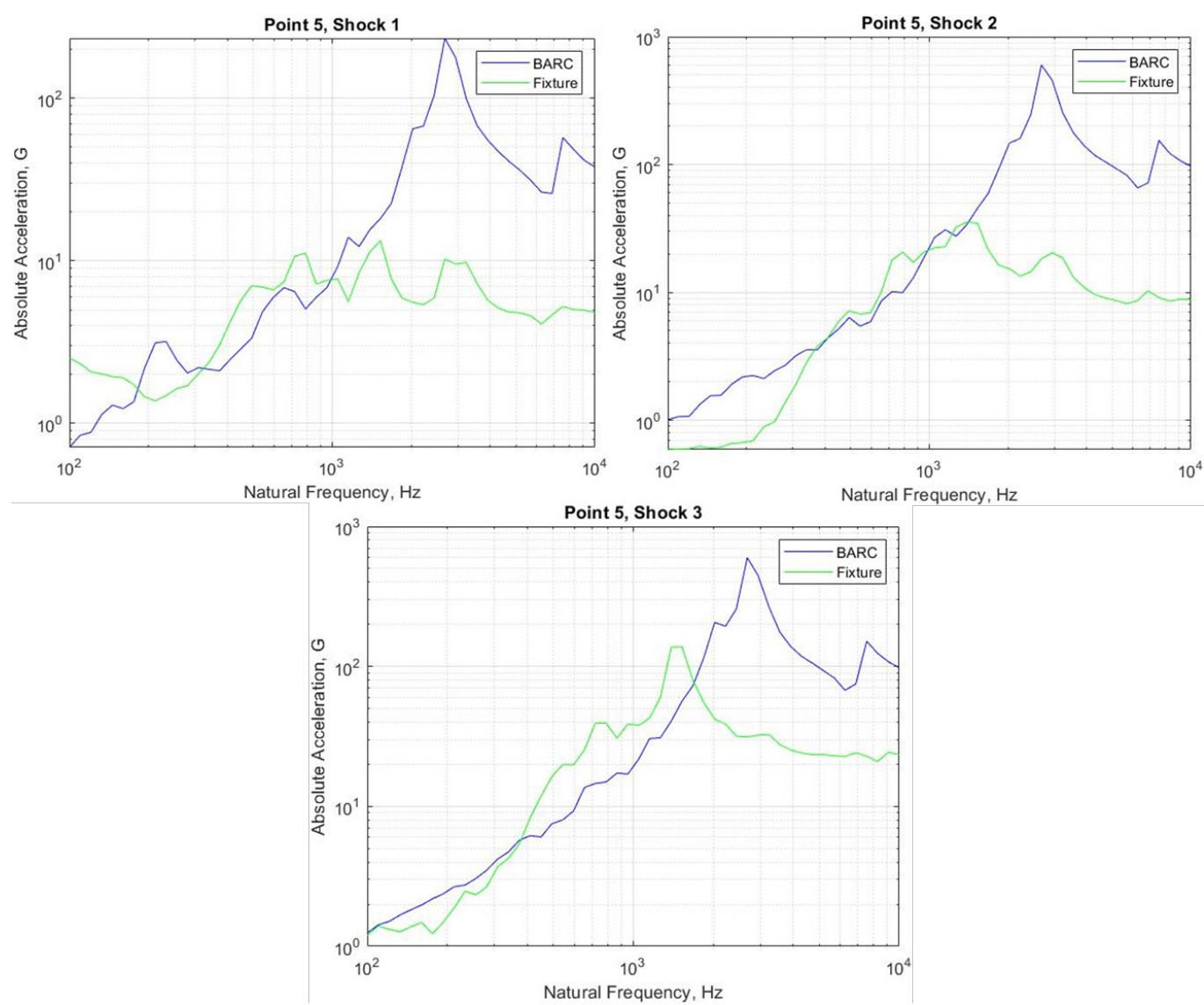

Figure 9.15 Fixed BARC vs. fixture SRS from point 5 on component for 3 identified shock events. 


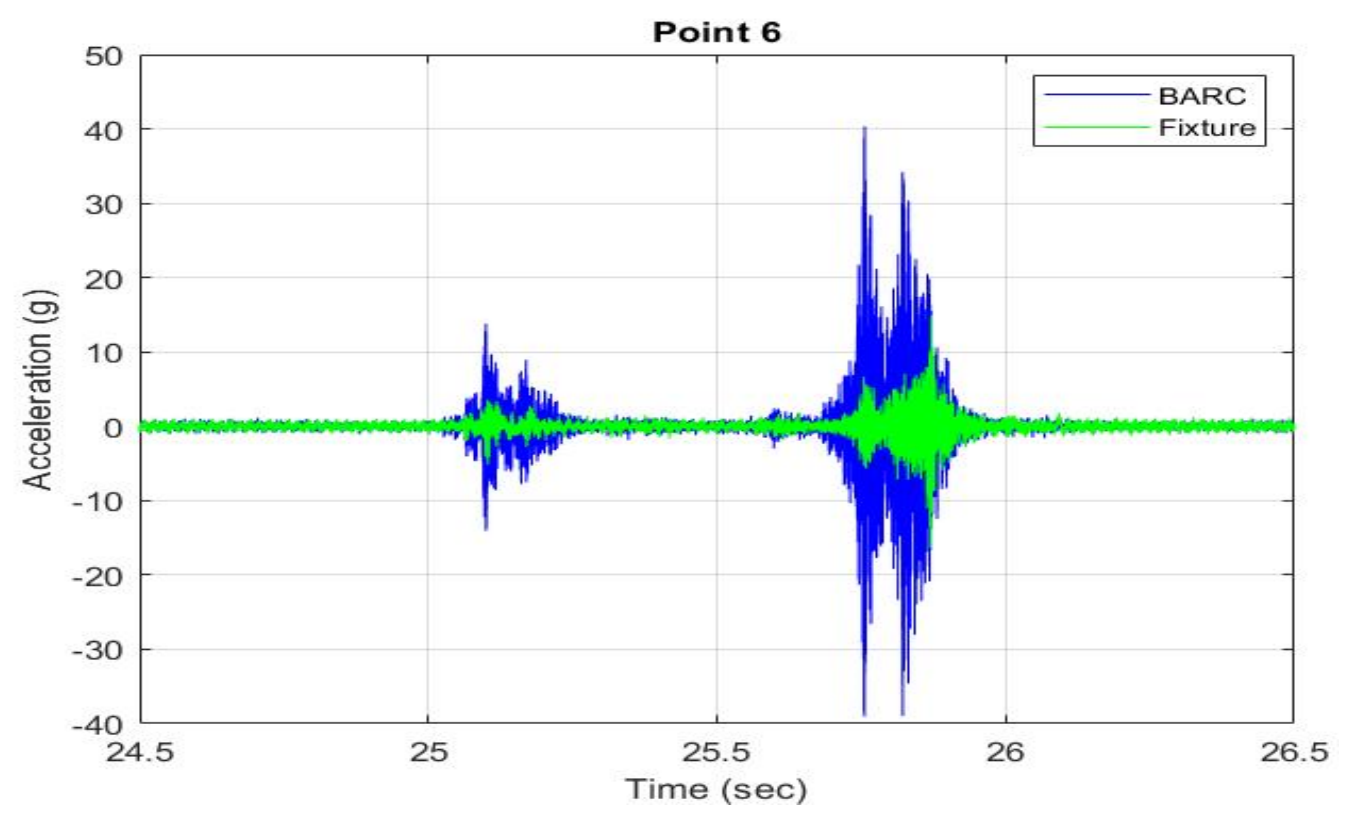

Figure 9.16 Component response comparison on fixture vs. BARC at point 6 with field data input.
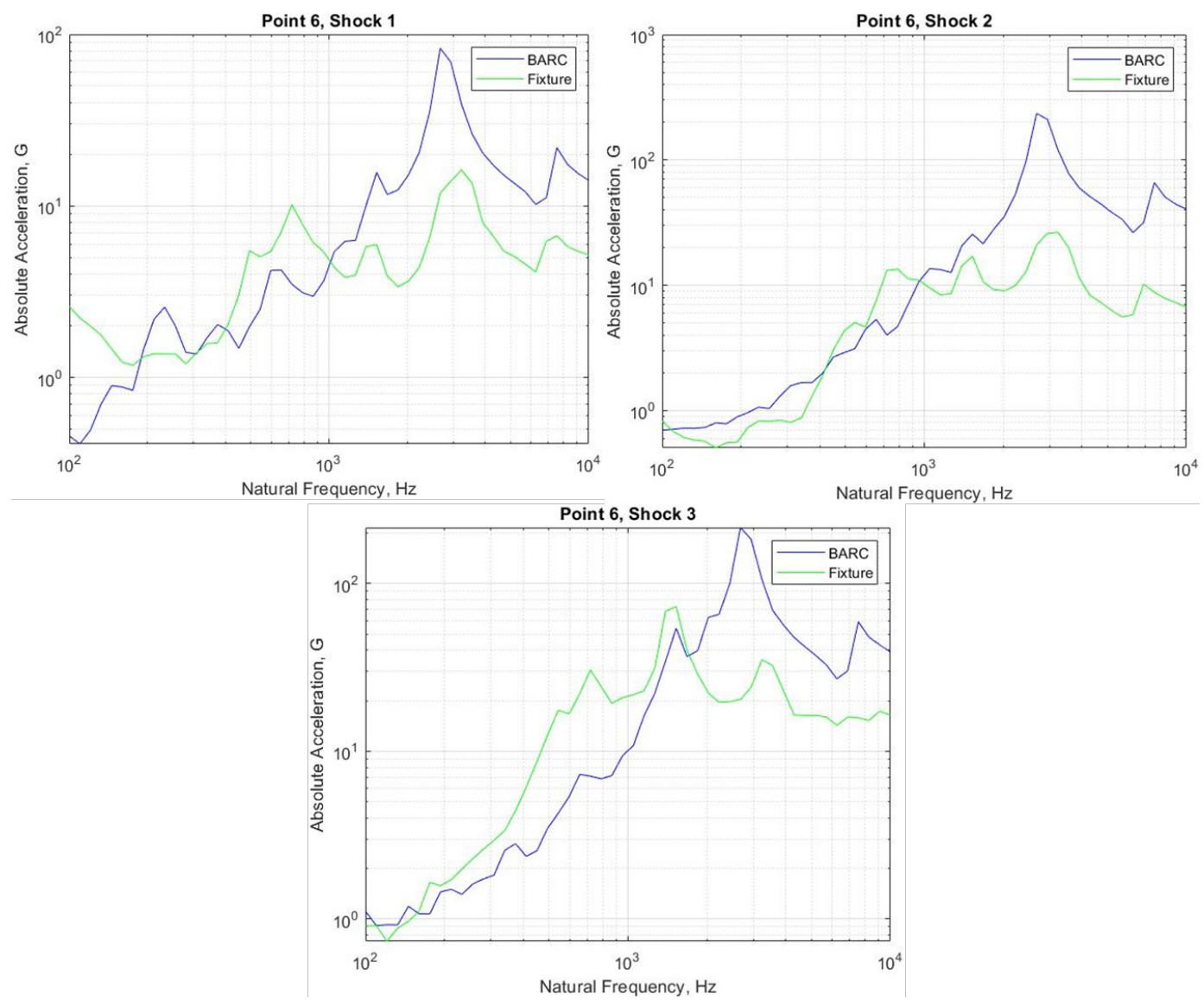

Figure 9.17 Fixed BARC vs. fixture SRS from point 6 on component for 3 identified shock events. 